\title{
PRESSURE CURVES FOR COMPRESSIBLE FLOW WITH SLIP THROUGH CONSTRICTED CYLINDERS
}

by

Salahaldeen Rabba

Bachelor of Electrical Engineering, Lakehead University, 2002

Diploma of Electrical Engineering, Lakehead University, 2000

\author{
A thesis \\ presented to Ryerson University \\ in partial fulfillment of the \\ requirements for the degree of \\ Master of Science \\ in the Program of \\ Applied Mathematics
}

Toronto, Ontario, Canada, 2014

(C)Salahaldeen Rabba 2014 


\section{Author's Declaration}

I hereby declare that I am the sole author of this thesis. This is a true copy of the thesis, including any required final revisions, as accepted by my examiners.

I authorize Ryerson University to lend this thesis to other institutions or individuals for the purpose of scholarly research.

I further authorize Ryerson University to reproduce this thesis by photocopying or by other means, in total or in part, at the request of other institutions or individuals for the purpose of scholarly research.

I understand that my thesis may be made electronically available to the public. 


\begin{abstract}
PRESSURE CURVES FOR COMPRESSIBLE FLOW WITH SLIP THROUGH CONSTRICTED CYLINDERS

Master of Science 2014

Salahaldeen Rabba

Applied Mathematics

Ryerson University
\end{abstract}

The goal of this research is to determine more accurate pressure curves for compressible flow with slip through cylinders that have a local constriction. Existing analyses for compressible flow with slip through a local constriction linearize the pressure gradient equation, and higher-order derivatives are dropped as well. The equation to be developed as part of this research retains quadratic pressure gradient terms. The corresponding solution for the pressure gradient is found following existing analysis in the literature for incompressible, no-slip flow, and subsequently the pressure is found using numerical integration. Results are compared to those from the linearized pressure gradient equation to see the extent of the improvement. Results are also assessed and compared to pressure and density curves available in the literature for some specific constrictions. Improvements may be obtained when the second-order derivatives are kept as well in the pressure gradient equations, which can be assessed and analyzed in the same context.

Pressure curves are assessed in different idealized flow geometries including the Gaussian model, a cosinusoidal model, and a smooth piece-wise polynomial model. In each case the pressure is found, and hence also the density. A range of Reynolds numbers, degrees of constriction, length of the constriction, compressibility and slip are considered.

Density curves are separately compared to existing particle-based results obtained from a stochastic particle dynamics using multiparticle collision dynamics. 


\section{Acknowledgements}

I am grateful to Dr. Katrin Rohlf for her continuous support and help. 


\section{Dedication}

I would like to dedicate this Thesis to my parents and Leanne Wilkins. 


\section{Contents}

1 Introduction $\quad 1$

2 Background $\quad 3$

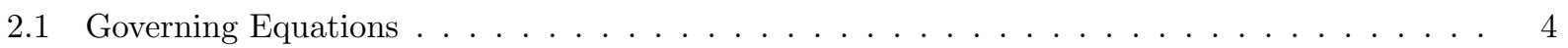

2.2 Axisymmetric flow geometries $\ldots \ldots \ldots \ldots \ldots \ldots \ldots \ldots \ldots$

3 Pressure Gradient $r$

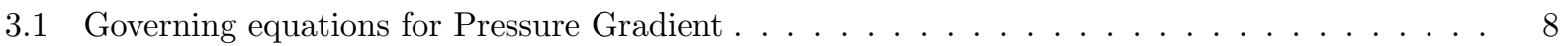

3.2 Axial velocity in terms of $d P / d z \ldots \ldots \ldots \ldots \ldots \ldots \ldots$

3.3 Pressure Gradient Equation . . . . . . . . . . . . . . . . . . . . . . 10

3.4 Pressure gradient and Pressure solution $\ldots \ldots \ldots \ldots \ldots \ldots \ldots$

4 Analysis and discussion $\quad 17$

4.1 Gaussian Geometry . . . . . . . . . . . . . . . . . . . 17

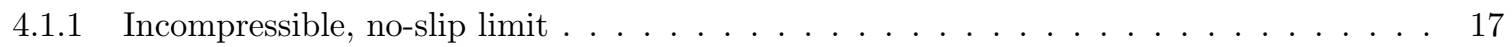

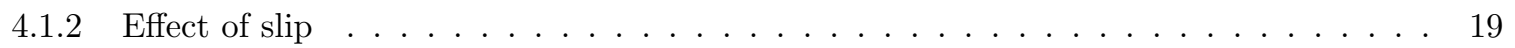

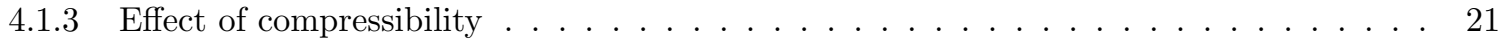

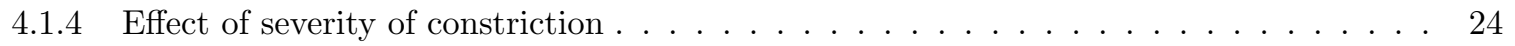

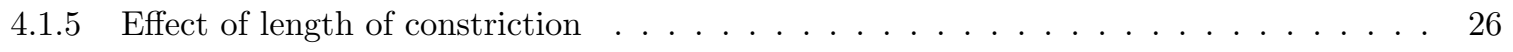

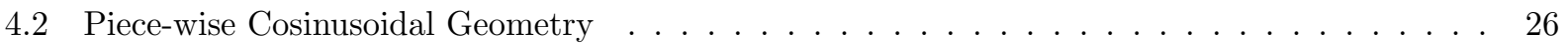

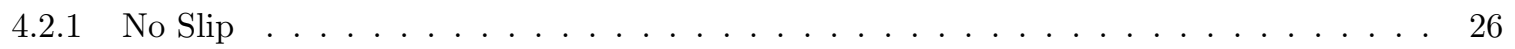

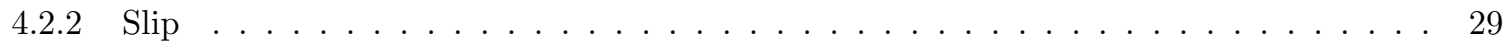

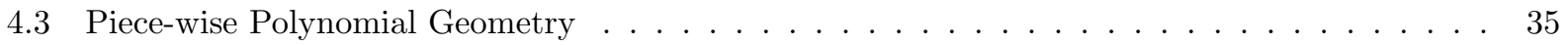

4.3.1 Comparison with MPC results and existing theoretical curves . . . . . . . . . . 35

5 Conclusions and Future work $\quad 40$

$\begin{array}{ll}\text { References } & 52\end{array}$ 


\section{List of Figures}

2.1 Flow Geometry for Gaussian Model _. . . . . . . . . . . . . . . . . . . . . 4

2.2 Flow Geometry for piece-wise polynomial model. This flow geometry can be made asymmetric in the axial direction by choosing values for $l_{1}$ and $l_{2}$ such that they are not equal. $\ldots \ldots 7$

4.1 Pressure gradient curves in Gaussian geometry with $\delta=0.1, b=0.25, w_{s}=0$, and $M a_{0}=$

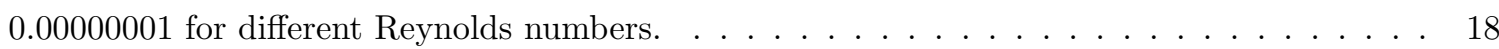

4.2 Pressure curves in Gaussian geometry with $\delta=0.1, b=0.25, w_{s}=0$, and $M a_{0}=0.00000001$

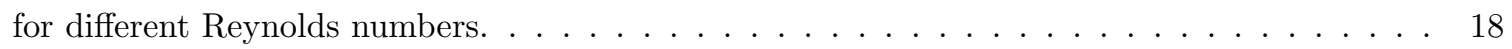

4.3 Pressure gradient in Gaussian geometry with $\delta=0.1, b=0.25, M a_{0}=0.00000001$ and

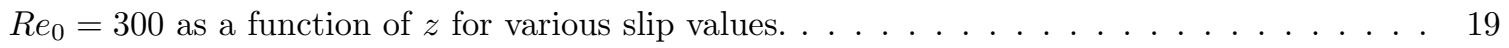

4.4 Pressure curves in Gaussian geometry with $\delta=0.1, b=0.25, M a_{0}=0.00000001$ and $R e_{0}=$

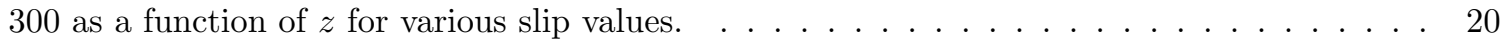

4.5 Pressure gradient in Gaussian geometry with $\delta=0.1, b=0.25, M a_{0}=0.1$ and $R e_{0}=300$ as

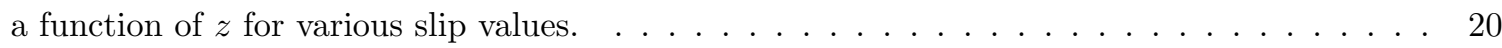

4.6 Pressure curves in Gaussian geometry with $\delta=0.1, b=0.25, M a_{0}=0.1$ and $R e_{0}=300$ as a

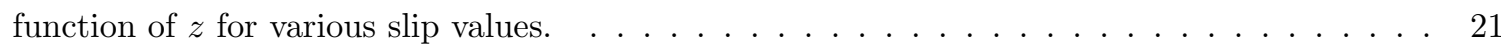

4.7 Pressure gradient in Gaussian geometry with $\delta=0.1, b=0.25, w_{s}=0$ and $R e_{0}=300$ as a function of $z$ for various $M a_{0}$ numbers. $\ldots \ldots \ldots \ldots \ldots$

4.8 Pressure curves in Gaussian geometry with $\delta=0.1, b=0.25, w_{s}=0$ and $R e_{0}=300$ as a

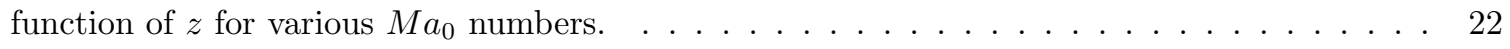

4.9 Pressure gradient curves in Gaussian geometry with $\delta=0.1, b=0.25, w_{s}=0.1$ and $\operatorname{Re}_{0}=300$

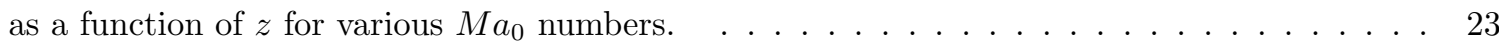

4.10 Pressure curves in Gaussian geometry with $\delta=0.1, b=0.25, w_{s}=0.1$ and $R e_{0}=300$ as a

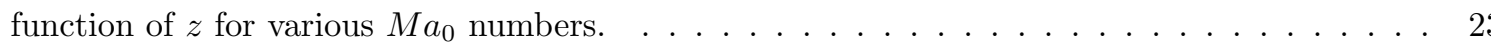

4.11 Pressure gradient curves in Gaussian geometry with $b=0.25, w_{s}=0, M a_{0}=0.1$ and

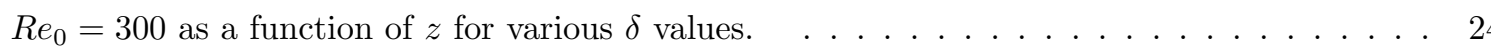

4.12 Pressure curves in Gaussian geometry with $b=0.25, w_{s}=0, M a_{0}=0.1$ and $R e_{0}=300$ as a

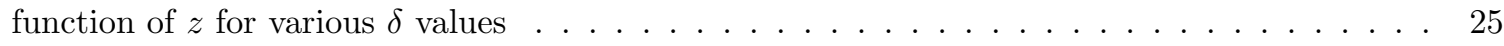

4.13 Pressure gradient curves in Gaussian geometry with $b=0.25, w_{s}=0.1, M a_{0}=0.1$ and $R e_{0}=300$ as a function of $z$ for various $\delta$ values. 
4.14 Pressure curves in Gaussian geometry with $b=0.25, w_{s}=0.1, M a_{0}=0.1$ and $R e_{0}=300$ as a function of $z$ for various $\delta$ values . . . . . . . . . . . . . . . . . . . . . 26

4.15 Pressure gradient curves in Gaussian geometry with $\delta=0.1, w_{s}=0, M a_{0}=0.1$ and $R e_{0}=300$ as a function of $z$ for various $b$ values. . . . . . . . . . . . . . . . . . 27

4.16 Pressure curves in Gaussian geometry with $\delta=0.1, w_{s}=0, M a_{0}=0.1$ and $R e_{0}=300$ as a function of $z$ for various $b$ values . . . . . . . . . . . . . . . . . . . . . . 27

4.17 Pressure gradient curves in Gaussian geometry with $\delta=0.1, w_{s}=0.1, M a_{0}=0.1$ and $R e_{0}=300$ as a function of $z$ for various $b$ values. . . . . . . . . . . . . . . . 28

4.18 Pressure curves in Gaussian geometry with $\delta=0.1, w_{s}=0.1, M a_{0}=0.1$ and $R e_{0}=300$ as a function of $z$ for various $b$ values . . . . . . . . . . . . . . . . . . . . . . . . . . . . . 28

4.19 Pressure gradient curves in Piece-wise Cosinusoidal Geometry with $\delta=0.1, w_{s}=0$ and $M a_{0}=0.0000001$ as a function of $z$ for various Reynolds numbers. . . . . . . . . . . . . 29

4.20 Pressure curves in Piece-wise Cosinusoidal Geometry with $\delta=0.1, w_{s}=0$ and $M a_{0}=$ 0.0000001 as a function of $z$ for various Reynolds numbers. . . . . . . . . . . . . .

4.21 Pressure gradient curves in Piece-wise Cosinusoidal Geometry with $\delta=0.2, w_{s}=0$ and $M a_{0}=0.0000001$ as a function of $z$ for various Reynolds numbers. . . . . . . . . . . .

4.22 Pressure curves in Piece-wise Cosinusoidal Geometry with $\delta=0.2, w_{s}=0$ and $M a_{0}=$ 0.0000001 as a function of $z$ for various Reynolds numbers. . . . . . . . . . . . . . . . . 31

4.23 Pressure gradient curves in Piece-wise Cosinusoidal Geometry with $\delta=0.3, w_{s}=0$ and $M a_{0}=0.0000001$ as a function of $z$ for various Reynolds numbers. . . . . . . . . . .

4.24 Pressure curves in Piece-wise Cosinusoidal Geometry with $\delta=0.3, w_{s}=0$ and $M a_{0}=$ 0.0000001 as a function of $z$ for various Reynolds numbers. . . . . . . . . . . . . . . . 32

4.25 Pressure gradient curves in Piece-wise Cosinusoidal Geometry with $\delta=0.3, w_{s}=0.1$ and $M a_{0}=0.0000001$ as a function of $z$ for various Reynolds numbers. . . . . . . . . . .

4.26 Pressure curves in Piece-wise Cosinusoidal Geometry with $\delta=0.3, w_{s}=0.1$ and $M a_{0}=$ 0.0000001 as a function of $z$ for various Reynolds numbers

4.27 Pressure gradient curves in Piece-wise Cosinusoidal Geometry with $\delta=0.3, w_{s}=0.1$ and $M a_{0}=0.1$ as a function of $z$ for various Reynolds numbers. . . . . . . . . . . . . .

4.28 Pressure curves in Piece-wise Cosinusoidal Geometry with $\delta=0.3, w_{s}=0.1$ and $M a_{0}=0.1$ as a function of $z$ for various Reynolds numbers. . . . . . . . . . . . . . . . . . . . . . . 34

4.29 My Pressure curves in Piece-wise Polynomial Geometry with $\delta=0.5, l_{1}=20, l_{2}=10, w_{s}=0$ and $g=0.01$ as a function of $z$ and Comparison with MPC results and theoretical curves

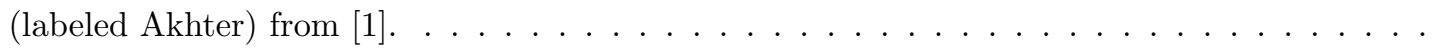

4.30 My Pressure curves in Piece-wise Polynomial Geometry with $\delta=0.5, l_{1}=20, l_{2}=10, w_{s}=0$ and $g=0.02$ as a function of $z$ and Comparison with Akhter's MPC results and her theoretical

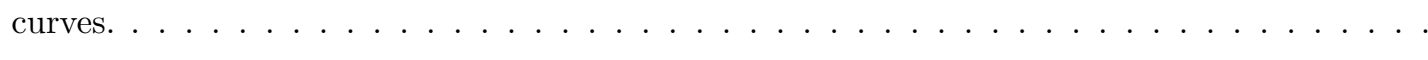

4.31 My Pressure curves in Piece-wise Polynomial Geometry with $\delta=0.5, l_{1}=20, l_{2}=10$, $w_{s}=0$ and $g=0.005$ as a function of $z$ and Comparison with Akhter's MPC results and her theoretical curves. . . . . . . . . . . . . . . . . . . . . . . . . . 
4.32 My Pressure curves in Piece-wise Polynomial Geometry with $\delta=0.5, l_{1}=20, l_{2}=10$, $w_{s}=0.5$ and $g=0.01$ as a function of $z$ and Comparison with Akhter's MPC results and her

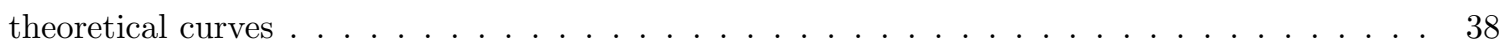

4.33 My Pressure curves in Piece-wise Polynomial Geometry with $\delta=0.5, l_{1}=20, l_{2}=10$, $w_{s}=0.5$ and $g=0.02$ as a function of $z$ and Comparison with Akhter's MPC results and her

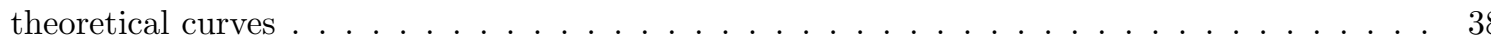

4.34 My Pressure curves in Piece-wise Polynomial Geometry with $\delta=0.5, l_{1}=20, l_{2}=10$, $w_{s}=0.5$ and $g=0.005$ as a function of $z$ and Comparison with Akhter's MPC results and her theoretical curves . . . . . . . . . . . . . . . . . . . . . . . . . 


\section{List of Appendices}

Appendix A

Appendix B

Appendix C

Appendix D 


\section{Chapter 1}

\section{Introduction}

When blood vessels are constricted then blood flow through those vessels becomes restricted. This means that less blood flows through those vessels feeding organs. Constricted vessels indicate a higher blood pressure. A mathematical model is developed analytically to study the flow characteristics of blood through an artery in the presence of stenosis (constriction). An appropriate shape of stenoses of arterial narrowing caused is constructed mathematically. The artery is simulated as a cylindrical tube having a narrowed portion forming stenoses. The viscosity of blood is taken to be constant. The flow mechanism in the stenosed artery is subjected to a pulse-pressure-gradient. Finally, the extensive quantitative analysis helps estimating the significant effects of the severity of the stenoses, effecting blood pressure and density.

Investigation of the flow in a stenosed (constricted) geometry is of interest because of its significance in biomechanics, especially in human vascular diseases [3]. When too much cholesterol or high levels of fatty substances or both are present in the blood, these fatty substances can begin to build fatty streaks along the inside lining of the artery wall and cause narrowing of the flow channel. This thickening of an artery wall, and subsequent potential blockage or rupture of the artery due to higher than normal pressures, is understood to be one of the leading causes of human death. Thus, the behaviour of the blood pressure in arterial stenoses bears some important aspects both due to engineering interest as well as feasible medical applications.

In order to understand the effect of stenosis on blood pressure through and beyond the constricted region of the artery, idealized flow geometries in the form of cylinders are often employed. This has been the subject of much research numerically, one such work being [15]. Numerical simulation of steady flow through axially symmetric stenoses, have been reported by several investigators, among which are [8] and $[6,19,20]$. Li [9] also had a similar study which was based on blood flow through arteries where Reynolds numbers varied from 50 to 750 for medium to large sized cylinders, Developing an overall understanding of the flow properties through a local constriction can also be important for early diagnosis, prevention and treatment of vascular disease $[11,10]$.

Although most analyses treat blood as an incompressible Newtonian fluid obeying the no-slip boundary condition at the walls, some studies have considered slip [16, 2, 1, 17] and some have allowed compressibility $[4,2]$. With the recent rise in popularity for using particle-based methods for blood flow applications [22, 
$5,2,1]$, compressibility needs to be addressed as particle-based methods are compressible in nature. Beside these, taking the non-Newtonian properties of the blood into consideration, there have been numerous studies simulating the blood flow through stenoses by incorporating an incompressible non-Newtonian model. The geometry corresponds to a cylindrical tube with a partial occlusion. Numerous studies have also investigated the effect of pulsation and other properties of blood. Finally, various mathematical models have been developed by a number of researchers $[12,13,14,16,18]$.

Many researchers have studied flow through stenosis from an analytical perspective. One popular approach is to use a Karman-Pohlhausen method for incompressible Newtonian flow through a local constriciton $[8,7]$. Compressible flow with slip was later incorporated, as well as slip at the wall [1]. In Akhter and Rohlf [1] the Karman-Pohlhausen method was used to develop the axial velocity distribution for steady, Newtonian flow through a constricted cylinder, allowing for slip at the wall as well as compressibility. The analysis is a natural extension of the original work in [8]. The flow geometry was a piecewise polynomial curve that allowed for asymmetry about the location of maximum constriction. Effects of compressibility, slip and flow geometry were assessed. Akhter and Rohlf [1] solved analytically the Navier-Stokes equations in a distribution for a flow through a channel with locally asymmetric constrictions and compared to simulation results for a particle-based flow that has compressibility built in.

The goal of this research is to further study the pressure and density curves generated by Akhter and Rohlf [1] and to determine more accurate pressure curves for compressible flow with slip though cylinders that have a local constriction. Existing analysis for compressible flow with slip through a local constriction leads to the pressure gradient equation, and higher-order derivatives and nonlinear pressure gradient terms are generally dropped. The equation that is developed in this Thesis retains quadratic pressure gradient terms. The corresponding solution for the pressure gradient is found which is solved for the pressure through numerical integration. Results are compared to existing pressure gradient and pressure curves in the incompressible, no-slip case, in particular flow geometries used in the original works, to see the extent of the improvement. Results are also assessed separately for distinctive features and compared to pressure curves available in the literature when possible. Improvements obtained when second-order derivatives are kept as well in the pressure gradient equations will be a future extended-study, where the second-order derivatives can be assessed and analyzed in the same context. We provide the equations with the second derivative terms, but drop them as a first approximation. We consider three flow geometries: the Gaussian (or bell-shaped) model, a piecewise cosinusoidal geometry, and a piecewise polynomial form. The dimensionless diameter constriction was allowed to vary from 0.1 to 0.4 for the bell-shaped constriction and the dimensionless stenotic degree was varied from 0.1 to 0.4 . Pressure gradient and pressure curves are presented in the different flow geometries for various Reynolds numbers, various severities of the constriction, various values for slip, and various compressibility.

This Thesis is organized as follows: In Chapter 2 we provide the Fluid Dynamics background that is required for our analytical results as well as the mathematical forms for the various flow geometries considered in our later assessment of the pressure curves. Chapter 3 provides a derivation/calculation approach of the pressure gradient equation. Chapter 4 contains a discussion and analysis of our results. Finally, chapter 5 provides important conclusions and future work. 


\section{Chapter 2}

\section{Background}

Fluid dynamics deals with fluid flow, the natural science of fluids (liquids and gases) in motion. Fluid dynamics has a wide range of applications, including calculating forces and momentum, and determining the mass flow rate of liquids through cylinders. Fluid dynamics offers a systematic structure, which underlies the laws derived from flow measurement, and is used to solve practical problems. The solution to a fluid dynamics problem typically involves calculating various properties of the fluid, such as velocity, pressure, density, and temperature, as functions of space. These will play a key role in our approach in deriving the pressure gradient equation and analysing the pressure and density curves. The concept of pressure is central to the study of this research. A pressure can be identified for every point in a body of the cylinder before, within and after the constriction.

The foundational laws of fluid dynamics are conservation laws, specifically, conservation of mass, conservation of linear momentum (also known as Newton's Second Law of Motion), and conservation of energy (also known as the First Law of Thermodynamics). In addition to the these, fluids are assumed to obey the continuum assumption. Fluids are composed of molecules that collide with one another. However, the continuum assumption does not consider fluids at this level, but rather at a scale in which the collective effects of many molecules contained in a 'fluid element' is described in a continuous manner. Consequently, in our research properties such as density, pressure, and velocity are assumed to be continuous.

The conservation equations for Newtonian fluids are called the NavierStokes equations of motion, which are a non-linear system of differential equations. The Navier-Stokes equations describe the motion of fluid substances, that is substances which can flow. These equations arise from applying Newton's second law to fluid motion, together with the assumption that the fluid stress is proportional to the gradient of velocity. For compressible flow, there is an additional equation of state that provides a relationship between pressure and density. All fluids are compressible to some extent, that is, changes in pressure will result in changes in density.

In this chapter, the governing compressible Navier-Stokes equations of motion are provided in general vector form, as well as in cylindrical coordinates under the steady, axisymmetric flow assumption. Additionally, the equation of state is chosen to be that for an ideal gas. These equations will be used in the 


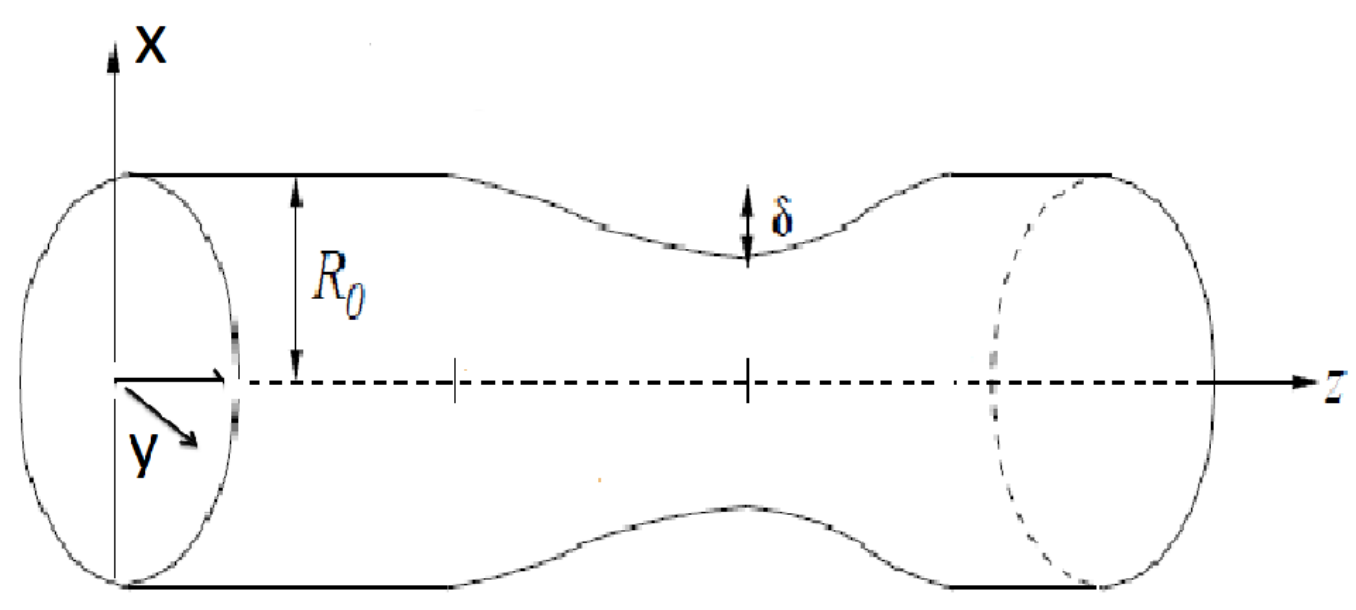

Figure 2.1: Flow Geometry for Gaussian Model

next chapter to derive the pressure gradient equation. Additionally, the various flow geometries that will be considered in this Thesis are presented in the last section of this chapter.

\section{Flow Geometry}

The geometry configuration of the cylinder tube with a constriction and its coordinate system is shown in Figure 2.1. The coordinate variables $(r, z)$ are defined in the cylinder coordinate system; $R_{o}$ is the radius of the cylinder having a constant cross section and $\delta$ determines the degree of the constriction. The study of flow geometry of the constriction can be described by the bell-shaped Gaussian distribution profile.

\subsection{Governing Equations}

The governing equations of motion for a compressible, isothermal, viscous flow of an ideal gas are given by

$$
\begin{aligned}
& \frac{\partial \rho}{\partial t}+\nabla \cdot(\rho \mathbf{u})=0 \text { (conservation of mass) } \\
& \rho \frac{D \mathbf{u}}{D t}=-\nabla P+\rho \mathbf{f}+\mu \nabla^{2} \mathbf{u}+\lambda \nabla(\nabla \cdot \mathbf{u}) \quad \text { (conservation of momentum) } \\
& P=\frac{k_{B} T}{m} \rho, \text { (equation of state) }
\end{aligned}
$$


where $\rho$ is the density, $t$ is time, $\frac{D}{D t}=\frac{\partial}{\partial t}+\mathbf{u} \cdot \nabla$ is the material derivative, $\nabla=\left(\frac{\partial}{\partial x}, \frac{\partial}{\partial y}, \frac{\partial}{\partial z}\right)$ is the gradient operator with spatial coordinates $(x, y, z), \mathbf{u}$ is the velocity vector, $P$ is the pressure, $\mathbf{f}$ corresponds to an external force, $\mu$ is the viscosity, $m$ is the mass of a fluid particle, $k_{B}$ is the Boltzmann constant, and $T$ is the constant fluid temperature.

Assuming steady-state and axisymmetry, the velocity vector in cylindrical coordinates is assumed to have the form

$$
\mathbf{u}=\left(u_{r}, u_{\theta}, u_{z}\right)=(u(r, z), 0, w(r, z)),
$$

together with $\rho=\rho(r, z)$. The governing equations, with an external force in the form $\mathbf{f}=\left(f_{r}, f_{\theta}, f_{z}\right)=$ $(0,0, \rho g)$ then become

$$
\begin{gathered}
\frac{\partial}{\partial r}(\rho u)+\frac{\partial}{\partial z}(\rho w)+\frac{\rho u}{r}=0 \text { (mass) } \\
\rho\left(u \frac{\partial u}{\partial r}+w \frac{\partial u}{\partial z}\right)=-\frac{\partial P}{\partial r}+\mu\left(\frac{\partial^{2} u}{\partial r^{2}}+\frac{1}{r} \frac{\partial u}{\partial r}+\frac{\partial^{2} u}{\partial z^{2}}-\frac{u}{r^{2}}\right)+\frac{\mu}{3} \frac{\partial}{\partial r}(\nabla \cdot \mathbf{u}),(r \text {-momentum }) \\
\rho\left(u \frac{\partial w}{\partial r}+w \frac{\partial w}{\partial z}\right)=\rho g-\frac{\partial P}{\partial z}+\mu\left(\frac{\partial^{2} w}{\partial r^{2}}+\frac{1}{r} \frac{\partial w}{\partial r}+\frac{\partial^{2} w}{\partial z^{2}}\right)+\frac{\mu}{3} \frac{\partial}{\partial z}(\nabla \cdot \mathbf{u}),(z \text {-momentum }) \\
P(r, z)=\frac{k_{B} T}{m} \rho(r, z), \text { (equation of state) }
\end{gathered}
$$

where

$$
\nabla \cdot \mathbf{u}=\frac{u}{r}+\frac{\partial u}{\partial r}+\frac{\partial w}{\partial z}
$$

and the $\theta$-momentum equation is identically satisfied.

\subsection{Axisymmetric flow geometries}

The flow characteristics in a constriction are studied for physiologically meaningful flow geometries for blood. Several key flow geometries are considered in the literature for such applications, and are assessed in this Thesis.

One popular flow geometry is the Gaussian geometry, Figure 2.1, where the radius is defined as a function of the longitudinal coordinate $z$ as follows:

$$
R(z)=R_{0}\left(1-\delta e^{-b z^{2}}\right) \quad(\text { Gaussian geometry) }
$$

In this description, $R_{0}$ represents the constricted radius far upstream and downstream of the constriction, $\delta$ determines the degree of the constriction, and $b$ controls the length.

Another popular model is one using a piece-wise defined function where the constriction is modelled with a cosinusoidal function. This geometry is defined as

$$
R(z)= \begin{cases}R_{0}, & \text { for } z \leq-z_{0} \text { and } z \geq z_{0} \\ R_{0}\left[1-\frac{\delta}{2}\left(1+\cos \frac{\pi z}{z_{0}}\right)\right] & \text { for }-z_{0} \leq z \leq z_{0}\end{cases}
$$


Again, $R_{0}$ is the upstream radius, $\delta$ controls the severity of the constriction, and $z_{0}$ is the half-length of the constriction.

Finally, a piece-wise cubic polynomial geometry is considered, and it is defined by

$$
R(z)= \begin{cases}R_{0} & z \leq z_{1} \\ a z^{3}+b z^{2}+c z+d & z_{1} \leq z \leq z_{2} \\ e z^{3}+f z^{2}+g z+h & z_{2} \leq z \leq z_{3} \\ R_{0} & z \geq z_{3}\end{cases}
$$

where the parameters are defined as follows:

$$
\begin{aligned}
& \delta \text { is the height of constriction } \\
& R_{0} \text { is the radius before and after the constriction } \\
& z_{1} \text { is start of the constriction } \\
& z_{2} \text { is the location of maximum constriction } \\
& z_{3} \text { is the end of the constriction } \\
& l_{1} \text { is the distance between } z_{1} \text { and } z_{2} \\
& l_{1}+l_{2} \text { is the length of the constriction }
\end{aligned}
$$

The values of $a-h$ are determined by imposing continuity and differentiability at $z=z_{1}, z_{2}$ and $z_{3}$, and imposing a local minimum at $z=z_{2}$ with height $R_{0}-\delta$. They are:

$$
\begin{aligned}
a & =\frac{2 \delta}{l_{1}{ }^{2}} \\
b & =-\frac{3 \delta\left(2 y_{1}+l_{1}\right)}{l_{1}{ }^{3}} \\
c & =\frac{6 \delta y_{1}\left(y_{1}+l_{1}\right)}{l_{1}{ }^{3}} \\
d & =-\frac{2 \delta y_{1}{ }^{3}+3 \delta y_{1}{ }^{2} l_{1}-R_{o} l_{1}{ }^{3}}{l_{1}{ }^{3}} \\
e & =-\frac{2 \delta}{l_{2}{ }^{3}} \\
f & =\frac{3\left(2 y_{1}+2 l_{1}+l_{2}\right) \delta}{l_{2}{ }^{3}} \\
g & =-\frac{6 \delta\left(y_{1}{ }^{2}+2 y_{1} l_{1}+l_{1}{ }^{2}+y_{1} l_{2}+l_{1} L_{2}\right)}{l_{2}{ }^{3}} \\
h & =\frac{3 \delta l_{1}{ }^{2} l_{2}+3 \delta y_{1}{ }^{2} l_{2}+6 \delta y_{1} l_{1} l_{2}+2 \delta l_{1}{ }^{3}+2 \delta y_{1}{ }^{3}+6 \delta y_{1}{ }^{2} l_{1}+R_{o} l_{2}{ }^{3}-\delta l_{2}{ }^{3}}{l_{2}{ }^{3}}
\end{aligned}
$$

This particular flow geometry is shown in Figure 2.2. Unlike the Gaussian and cosinusoidal model, this flow geometry can be made asymmetric in the axial direction by choosing values for $l_{1}$ and $l_{2}$ such that they are not equal. 


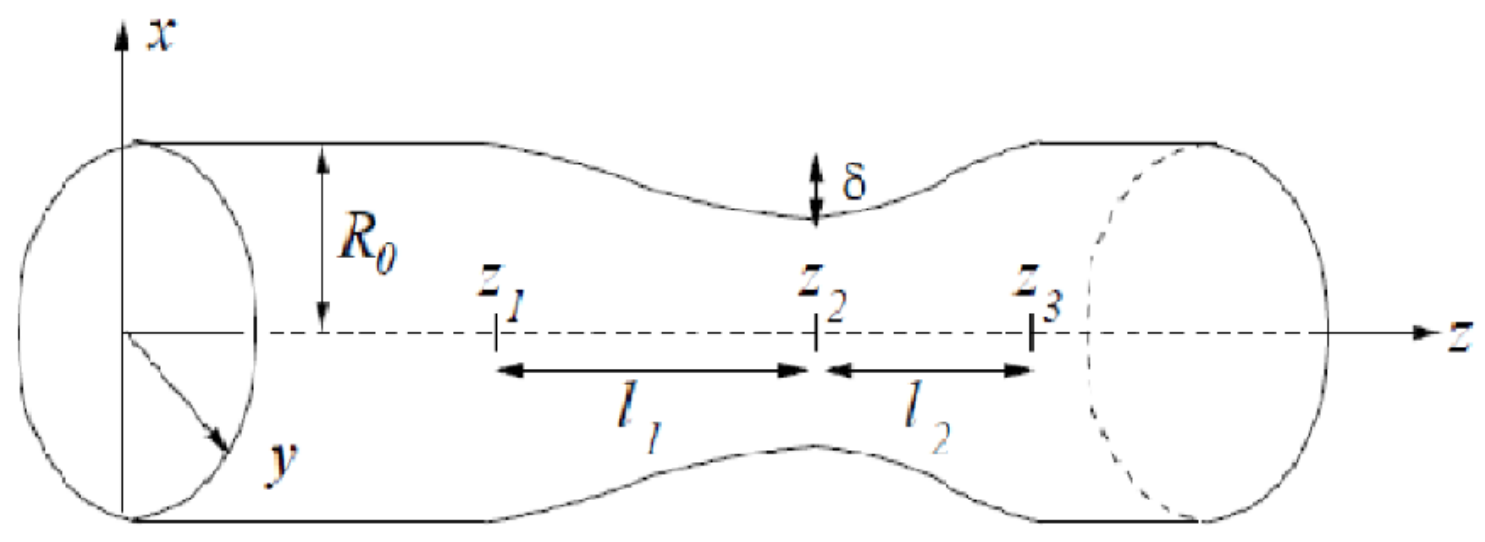

Figure 2.2: Flow Geometry for piece-wise polynomial model. This flow geometry can be made asymmetric in the axial direction by choosing values for $l_{1}$ and $l_{2}$ such that they are not equal. 


\section{Chapter 3}

\section{Pressure Gradient}

\subsection{Governing equations for Pressure Gradient}

As per Forrester and Young [9], for a mild constriction geometry the $r$-momentum equation (2.6) can be approximated as $\frac{\partial P}{\partial r}=0$, in which case (2.8) implies $\rho=\rho(z)$ which can be used in (2.5) to give

$$
\frac{u}{r}+\frac{\partial u}{\partial r}=-\frac{1}{\rho} \frac{\partial}{\partial z}(\rho w)
$$

Using this in the last term of (2.7), together with the assumption that $u \frac{\partial w}{\partial r} \ll w \frac{\partial w}{\partial z}$ allows us to write the system for determining $w(r, z)$ and $P(z)$ as

$$
\begin{gathered}
\rho w \frac{\partial w}{\partial z}=\rho g-\frac{d P}{d z}+\mu\left(\frac{\partial^{2} w}{\partial r^{2}}+\frac{1}{r} \frac{\partial w}{\partial r}+\frac{4}{3} \frac{\partial^{2} w}{\partial z^{2}}\right)-\frac{\mu}{3} \frac{\partial}{\partial z}\left(\frac{1}{\rho} \frac{\partial}{\partial z}(\rho w)\right) \\
P(z)=\frac{k_{B} T}{m} \rho(z), \quad \frac{d P}{d z}=\frac{k_{B} T}{m} \frac{d \rho}{d z} .
\end{gathered}
$$

Note: We have neglected the $u \frac{\partial w}{\partial r}$ term as flow in the radial direction $(u)$ is assumed to be negligible compared to the flow in the $z$ direction $(w)$, and changes of flow in the $z$ direction depends on changes in $z$ more than changes in $r$. Therefore, we can use the assumption that $u \frac{\partial w}{\partial r} \ll w \frac{\partial w}{\partial z}$, and thus we neglect $u \frac{\partial w}{\partial r}[9]$.

\subsection{Axial velocity in terms of $d P / d z$}

Following [9] and [1], we now assume that the radial dependence of the axial velocity $w$ is a fourth-order polynomial in the form

$$
\frac{w(r, z)}{W}=A \eta+B \eta^{2}+C \eta^{3}+D \eta^{4}+E
$$


where $\eta=\frac{R-r}{R}$, and $W=W(z)$ is the as yet unknown centerline velocity.

Constants $A$ to $E$ are determined by imposing:

(i) $w=\frac{w_{s}}{\sqrt{1+R^{\prime 2}}}$ at $r=R$ (slip boundary condition)

(ii) $\frac{\partial w}{\partial r}=0$ at $r=0$ (axisymmetric flow),

(iii) $w=W$ at $r=0$ (by definition of centerline velocity $W$ ),

(iv) $\frac{\partial^{2} w}{\partial r^{2}}=-\frac{2\left(W-w_{s}\right)}{R^{2}}$ at $r=0$ (nearly parabolic flow with slip),

(v) $\frac{d P}{d z} \approx \rho g+\mu\left(\frac{\partial^{2} w}{\partial r^{2}}+\frac{1}{r} \frac{\partial w}{\partial r}\right)$ at $r=R$ ( using (3.2))

The boundary conditions for slip at the wall is imposed by (i), and axisymmetric flow by (ii).

Condition (i) follows from solving $\mathbf{u} \cdot \mathbf{n}=0$ (vanishing normal component of velocity) and $\mathbf{u} \cdot \mathbf{t}=w_{s}$ (tangential component of velocity has magnitude $w_{s}$ ) for $w$, while (iv) comes from the velocity profile for parabolic flow with slip at the wall.

Imposing (i)-(v) and solving for the unknown constants gives

$$
\begin{gathered}
A=\frac{1}{7}\left(-\lambda+10-12 E+T+2 \frac{w_{s}}{W}\right) \\
B=\frac{1}{7}\left(3 \lambda+5-6 E-3 T+\frac{w_{s}}{W}\right) \\
C=\frac{1}{7}\left(-3 \lambda-12+20 E+3 T-8 \frac{w_{s}}{W}\right) \\
D=\frac{1}{7}\left(\lambda+4-9 E-T+5 \frac{w_{s}}{W}\right) \\
E=\frac{w_{s}}{W \sqrt{1+R^{\prime 2}}}
\end{gathered}
$$

where

$$
\lambda=\frac{R^{2}}{\mu W} \frac{d P}{d z}
$$

and

$$
T=\frac{\rho g R^{2}}{\mu W}
$$

Note that, since $P$ and $W$ are functions of $z$, so are $A-E$ in the axial velocity expression.

Some important results follow from this velocity distribution, that will be needed in the derivation of the pressure gradient equation in the next section. These are outlined in Appendix A and B. 


\subsection{Pressure Gradient Equation}

Starting from equation (3.2), namely,

$$
\rho w \frac{\partial w}{\partial z}=\rho g-\frac{d P}{d z}+\mu\left(\frac{\partial^{2} w}{\partial r^{2}}+\frac{1}{r} \frac{\partial w}{\partial r}+\frac{4}{3} \frac{\partial^{2} w}{\partial z^{2}}\right)-\frac{\mu}{3} \frac{\partial}{\partial z}\left(\frac{1}{\rho} \frac{\partial}{\partial z}(\rho w)\right)
$$

our goal is to construct an equation for $\frac{d P}{d z}$. This can be achieved as follows.

First, we multiply the equation by $\frac{r}{\rho}$ and then integrate across the cylinder over $r=0 \ldots R$. This leads to

$$
\int_{0}^{R} r w \frac{\partial w}{\partial z} d r=\int_{0}^{R} r g d r-\int_{0}^{R} \frac{r}{\rho} \frac{d P}{d z} d r+\int_{0}^{R} \frac{\mu}{\rho} r\left[\frac{\partial^{2} w}{\partial r^{2}}+\frac{1}{r} \frac{\partial w}{\partial r}+\frac{4}{3} \frac{\partial^{2} w}{\partial z^{2}}\right] d r-\int_{0}^{R} \frac{\mu}{3 \rho} r \frac{\partial}{\partial z}\left[\frac{1}{\rho} \frac{\partial}{\partial z}(\rho w)\right] d r .
$$

Recall that $\rho$ and $P$ are functions of $z$ only so that, for example, $\int_{0}^{R} r \frac{1}{\rho} \frac{d P}{d z} d r=\frac{1}{\rho} \frac{d P}{d z} \int_{0}^{R} r d r=\frac{1}{\rho} \frac{d P}{d z} \frac{R^{2}}{2}$. Thus we have

$$
\int_{0}^{R} r w \frac{\partial w}{\partial z} d r=\frac{g R^{2}}{2}-\frac{1}{\rho} \frac{d P}{d z} \frac{R^{2}}{2}+\int_{0}^{R}\left[\frac{\mu}{\rho}\left(\frac{4}{3} r \frac{\partial^{2} w}{\partial z^{2}}+r \frac{\partial^{2} w}{\partial r^{2}}+\frac{\partial w}{\partial r}-\frac{1}{3} r \frac{\partial}{\partial z}\left(\frac{1}{\rho} \frac{\partial}{\partial z}(\rho w)\right)\right] d r .\right.
$$

It follows that

$$
\frac{1}{2} \int_{0}^{R} r \frac{\partial}{\partial z} w^{2} d r=\frac{g R^{2}}{2}-\frac{1}{\rho} \frac{d P}{d z} \frac{R^{2}}{2}+\left.\frac{\mu R}{\rho}\left(\frac{\partial w}{\partial r}\right)\right|_{r=R}+\int_{0}^{R}\left[\frac{\mu}{\rho}\left(\frac{4}{3} r \frac{\partial^{2} w}{\partial z^{2}}-\frac{1}{3} r \frac{\partial}{\partial z}\left(\frac{1}{\rho} \frac{\partial}{\partial z}(\rho w)\right)\right] d r\right.
$$

since $r \frac{\partial^{2} w}{\partial r^{2}}+\frac{\partial w}{\partial r}=\frac{\partial}{\partial r}\left(r \frac{\partial w}{\partial r}\right)$.

Now the integral on the LHS can be rewritten using (B.3), from which we get

$$
\frac{1}{2} \int_{0}^{R} r \frac{\partial}{\partial z} w^{2} d r=\frac{1}{2} \frac{d}{d z} \int_{0}^{R} r w^{2} d r-\frac{1}{2} \frac{R R^{\prime} w_{s}^{2}}{\left(1+R^{\prime 2}\right)}
$$

The third term on the RHS of (3.15) can be simplified using the velocity distribution from the previous section. From (3.4)

$$
\begin{aligned}
\left.\frac{d w}{d r}\right|_{r=R} & =\left.\frac{d}{d r}\left[W\left(A \eta+B \eta^{2}+C \eta^{3}+D \eta^{4}+E\right)\right]\right|_{r=R} \\
& =\left.W\left[A \frac{d}{d r}\left(1-\frac{r}{R}\right)+B \frac{d}{d r}\left(1-\frac{r}{R}\right)^{2}+C \frac{d}{d r}\left(1-\frac{r}{R}\right)^{3}+D \frac{d}{d r}\left(1-\frac{r}{R}\right)^{4}+E \frac{d}{d r}(1)\right]\right|_{r=R} \\
& =\left.W\left[A\left(-\frac{1}{R}\right)+B\left(2\left(1-\frac{r}{R}\right)\left(-\frac{1}{R}\right)\right)+C\left(3\left(1-\frac{r}{R}\right)^{2}\left(-\frac{1}{R}\right)\right)+D\left(4\left(1-\frac{r}{R}\right)^{3}\left(-\frac{1}{R}\right)\right)+0\right]\right|_{r=R} \\
& =-\frac{W A}{R} .
\end{aligned}
$$

Substituting equations (3.16) and (3.17) in equation (3.15) and also rewriting the integral on the RHS, 
gives

$$
-\frac{1}{2} \frac{R R^{\prime} w_{s}^{2}}{1+R^{\prime 2}}+\frac{1}{2} \frac{d}{d z} \int_{0}^{R} r w^{2} d r=-\frac{1}{\rho} \frac{d P}{d z} \frac{R^{2}}{2}+\frac{g R^{2}}{2}+\nu R\left(-\frac{A W}{R}\right)+\frac{4}{3} \nu \int_{0}^{R} r \frac{d^{2} w}{d z^{2}} d r-\frac{\nu}{3} \int_{0}^{R} r \frac{d}{d z}\left[\frac{1}{\rho} \frac{d}{d z}(\rho w)\right] d r .
$$

Note that we have also used

$$
\nu=\frac{\mu}{\rho} .
$$

Solving (B.8) for $\int_{0}^{R} r \frac{d^{2} w}{d z^{2}} d r$, substituting on the RHS of (3.18), and using the product rule in the integrand in the last integral on the RHS gives

$$
\begin{gathered}
\frac{1}{2} \frac{d}{d z} \int_{0}^{R} r w^{2} d r=-\frac{1}{2} \frac{R R^{\prime} w_{s}^{2}}{1+R^{\prime 2}}-\frac{1}{\rho} \frac{d P}{d z} \frac{R^{2}}{2}+\frac{g R^{2}}{2}+\nu(-A W)+\frac{4}{3} \nu \frac{d^{2}}{d z^{2}} \int_{0}^{R} r w d r-\frac{4}{3} \nu \frac{R w_{s} R^{\prime \prime}}{\sqrt{1+R^{\prime 2}}}-\frac{4}{3} \nu \frac{w_{s} R^{\prime 2}}{\sqrt{1+R^{\prime 2}}} \\
+\frac{8}{3} \nu \frac{R R^{\prime 2} R^{\prime \prime} w_{s}}{\left(1+R^{\prime 2}\right)^{3 / 2}}-\frac{\nu}{3} \frac{1}{\rho} \int_{0}^{R} r \frac{d^{2}}{d z^{2}}(\rho w) d r+\frac{\nu}{3} \frac{1}{\rho^{2}} \frac{d \rho}{d z} \int_{0}^{R} r \frac{d}{d z}(\rho w) d r
\end{gathered}
$$

Using (B.8) and (B.2), the last two integrals on the RHS can be written in terms of $\frac{d^{2}}{d z^{2}} \int_{0}^{R} r \rho w d r$ and $\frac{d}{d z} \int_{0}^{R} r \rho w d r$ giving

$$
\begin{gathered}
\frac{1}{2} \frac{d}{d z} \int_{0}^{R} r w^{2} d r=-\frac{1}{2} \frac{R R^{\prime} w_{s}^{2}}{1+}+\frac{R^{\prime 2}}{\rho} \frac{1}{\rho} \frac{d P}{d z} \frac{R^{2}}{2}+\frac{g R^{2}}{2}+\nu(-A W)+\frac{4}{3} \nu \frac{d^{2}}{d z^{2}} \int_{0}^{R} r w d r-\frac{4}{3} \nu \frac{R w_{s} R^{\prime \prime}}{\sqrt{1+R^{\prime 2}}}-\frac{4}{3} \nu \frac{w_{s} R^{\prime 2}}{\sqrt{1+R^{\prime 2}}} \\
+\frac{8}{3} \nu \frac{R R^{\prime 2} R^{\prime \prime} w_{s}}{\left(1+R^{\prime 2}\right)^{3 / 2}}-\frac{\nu}{3} \frac{1}{\rho}\left[\frac{d^{2}}{d z^{2}} \int_{0}^{R} r \rho w d r-\frac{\rho\left(R R^{\prime \prime}+R^{\prime 2}\right)}{\sqrt{1+R^{\prime 2}}} w_{s}-\frac{2 R w_{s} R^{\prime}}{\sqrt{1+R^{\prime 2}}} \frac{d \rho}{d z}\right. \\
\left.+\frac{2 R \rho w_{s} R^{\prime 2} R^{\prime \prime}}{\left(1+R^{\prime 2}\right)^{3 / 2}}\right]+\frac{\nu}{3 \rho^{2}} \frac{d \rho}{d z}\left[\frac{d}{d z} \int_{0}^{R} r \rho w d r-\frac{R \rho w_{s} R^{\prime}}{\sqrt{1+R^{\prime 2}}}\right]
\end{gathered}
$$

For the next step it is helpful to note that, since $Q=\int_{0}^{R} r \rho w d r$ and since $\frac{d Q}{d z}=0$ (see Appendix A),

$$
\frac{d^{n}}{d z^{n}} \int_{0}^{R} r \rho w d r=0, \text { for } n \geq 1
$$

Thus, we have

$$
\begin{aligned}
\frac{1}{2} \frac{d}{d z} \int_{0}^{R} r w^{2} d r=-\frac{1}{2} \frac{R R^{\prime} w_{s}^{2}}{1+} & R^{\prime 2}-\frac{1}{\rho} \frac{d P}{d z} \frac{R^{2}}{2}+\frac{g R^{2}}{2}+\nu(-A W)+\frac{4}{3} \nu \frac{d^{2}}{d z^{2}} \int_{0}^{R} r w d r-\frac{4}{3} \nu \frac{R w_{s} R^{\prime \prime}}{\sqrt{1+R^{\prime 2}}}-\frac{4}{3} \nu \frac{w_{s} R^{\prime 2}}{\sqrt{1+R^{\prime 2}}} \\
& +\frac{8}{3} \nu \frac{R R^{\prime 2} R^{\prime \prime} w_{s}}{\left(1+R^{\prime 2}\right)^{3 / 2}}-\frac{\nu}{3} \frac{1}{\rho}\left[-\frac{\rho\left(R R^{\prime \prime}+R^{\prime 2}\right)}{\sqrt{1+R^{\prime 2}}} w_{s}-\frac{2 R w_{s} R^{\prime}}{\sqrt{1+R^{\prime 2}}} \frac{d \rho}{d z}+\frac{2 R \rho w_{s} R^{\prime 2} R^{\prime \prime}}{\left(1+R^{\prime 2}\right)^{3 / 2}}\right] \\
+ & \frac{\nu}{3 \rho^{2}} \frac{d \rho}{d z}\left[-\frac{R \rho w_{s} R^{\prime}}{\sqrt{1+R^{\prime 2}}}\right]
\end{aligned}
$$

At this point the equations become sufficiently messy that we leave out some of the specific equations and outline the method instead. 
Step 1: For the next step, obtain an expression for $\frac{d^{2}}{d z^{2}} \int_{0}^{R} r w d r$ as follows:

Using $\int_{0}^{R} r w d r=\frac{Q}{2 \pi \rho}$, with $\frac{d Q}{d z}=0$ to get an expression for $\frac{d^{2}}{d z^{2}} \int_{0}^{R} r w d r$, we have

$$
\begin{gathered}
\frac{d}{d z} \int_{0}^{R} r w d r=\frac{d}{d z}\left(\frac{Q}{2 \pi \rho}\right)=\frac{1}{2 \pi}\left[\frac{1}{\rho} \frac{d Q}{d z}-\frac{Q}{\rho^{2}} \frac{d \rho}{d z}\right]=-\frac{Q}{2 \pi \rho^{2}} \frac{d \rho}{d z} \\
\Rightarrow \frac{d^{2}}{d z^{2}} \int_{0}^{R} r w d r=\frac{d}{d z}\left[-\frac{Q}{2 \pi \rho^{2}} \frac{d \rho}{d z}\right]=-\rho^{2} \frac{d \rho}{d z} \frac{d Q}{d z}+2 Q \frac{1}{\rho^{3}}\left(\frac{d \rho}{d z}\right)^{2}-\frac{Q}{\rho^{2}} \frac{d^{2} \rho}{d z^{2}} .
\end{gathered}
$$

Thus

$$
\frac{d^{2}}{d z^{2}} \int_{0}^{R} r w d r=\frac{Q}{\pi \rho^{3}}\left(\frac{d \rho}{d z}\right)^{2}-\frac{Q}{2 \pi \rho^{2}} \frac{d^{2} \rho}{d z^{2}}
$$

Now write $Q$ in terms of $\bar{W}$ (see Appendix A equation (A.1)) and use the equation of state $P=\frac{k_{B} T}{m} \rho$ to write $\rho$ and any of its derivatives in terms of $P$. This gives

$$
\frac{4}{3} \nu \frac{d^{2}}{d z^{2}} \int_{0}^{R} r w d r=\frac{4}{3} \nu \frac{Q}{\pi \rho^{3}}\left(\frac{d \rho}{d z}\right)^{2}-\frac{4}{3} \nu \frac{Q}{2 \pi \rho^{2}} \frac{d^{2} \rho^{2}}{d z^{2}}=\frac{4}{3} \frac{\mu R^{2} \bar{W}}{\rho^{3}} \frac{m^{2}}{k_{B}^{2} T^{2}}\left(\frac{d P}{d z}\right)^{2}-\frac{2}{3} \frac{\mu R^{2} \bar{W}^{2}}{\rho^{2}} \frac{m}{k_{B} T} \frac{d^{2} P}{d z^{2}}
$$

Step 2: Next, use the expression for the axial velocity of the previous section to obtain an expression for $\nu(-A W)$ :

The $\nu(-A W)$ term is rewritten using $A$ from (3.5) and $W$ from (A.2). This leads to

$$
\begin{aligned}
-\nu(A R)=\frac{R^{2}}{7 \rho} \frac{d P}{d z} & -\frac{2100}{(7) 97} \nu \bar{W}-\frac{20}{(7)(97)} \frac{R^{2}}{\rho} \frac{d P}{d z}+\frac{20}{(7)(97)} R^{2} g+\frac{110}{(7)(97)} \nu w_{s} \\
& +\frac{1020}{(7)(97)} \nu \frac{w_{s}}{\sqrt{1+R^{\prime 2}}}+\frac{12}{7} \nu \frac{w_{s}}{\sqrt{1+R^{\prime 2}}}-\frac{1}{7} R^{2} g-\frac{2}{7} \nu w_{s}
\end{aligned}
$$

Step 3: Using equation (3.4) for $w$ we evaluate $\int_{0}^{R} r w^{2} d r$ using Maple and collect coefficients with respect to $\frac{d^{2} P}{d z^{2}},\left(\frac{d P}{d z}\right)^{2}, \frac{d P}{d z}$ and $\frac{d P}{d z}^{0}$ which we then differentiate to get $\frac{d}{d z} \int_{0}^{R} r w^{2} d r$. The result is (see Appendix C):

$$
\begin{aligned}
& \frac{d}{d z} \int_{0}^{R} r w^{2} d r=\frac{61}{263452} \frac{R^{5}\left(\frac{d P}{d z}\right)^{2} R^{\prime}}{\mu^{2}}+\frac{61}{790356} \frac{R^{6} \frac{d P}{d z}\left(\frac{d^{2} P}{d z^{2}}\right)}{\mu^{2}}-\frac{190}{197589} \frac{R^{\prime} w_{s} R^{3} \frac{d P}{d z}}{\mu} \\
& -\frac{95}{395178} \frac{w_{s} R^{4} \frac{d^{2} P}{d z^{2}}}{\mu}-\frac{3596}{197589} \frac{w_{s} R^{3} \frac{d P}{d z} R^{\prime}}{\mu \sqrt{1+R^{\prime 2}}}-\frac{899}{197589} \frac{w_{s} R^{4} \frac{d^{2} P}{d z^{2}}}{\mu \sqrt{1+R^{\prime 2}}}+\frac{899}{197589} \frac{w_{s} R^{4} \frac{d P}{d z} R^{\prime} R^{\prime \prime}}{\mu\left(1+R^{\prime 2}\right)^{3 / 2}} \\
& +\frac{631}{65863} \frac{R Q \frac{d P}{d z} R^{\prime}}{\mu \rho \pi}+\frac{631}{131726} \frac{R^{2} Q \frac{d^{2} P}{d z^{2}}}{\mu \rho \pi}-\frac{631}{131726} \frac{R^{2} Q \frac{d P}{d z} \frac{d \rho}{d z}}{\mu \rho^{2} \pi}-\frac{61}{131726} \frac{R^{5} \rho g \frac{d P}{d z} R^{\prime}}{\mu^{2}} \\
& -\frac{61}{790356} \frac{R^{6}\left(\frac{d \rho}{d z}\right) g \frac{d P}{d z}}{\mu^{2}}-\frac{61}{790356} \frac{R^{6} \rho g \frac{d^{2} P}{d z^{2}}}{\mu^{2}}+\frac{154937}{395178} \frac{w_{s} Q R^{\prime} R^{\prime \prime}}{\left(1+R^{\prime 2}\right)^{3 / 2} \rho \pi}+\frac{154937}{395178} \frac{w_{s} Q^{\frac{d \rho}{d z}}}{\sqrt{1+R^{\prime 2} \rho^{2} \pi}}
\end{aligned}
$$




$$
\begin{aligned}
& +\frac{376318}{987945} \frac{w_{s}^{2} R R^{\prime}}{1+R^{\prime 2}}-\frac{376318}{987945} \frac{w_{s}^{2} R^{2} R^{\prime} R^{\prime \prime}}{\left(1+R^{\prime 2}\right)^{2}}+\frac{899}{197589} \frac{w_{s} \frac{d \rho}{d z} R^{4} g}{\mu \sqrt{1+R^{\prime 2}}}+\frac{3596}{197589} \frac{w_{s} \rho R^{3} g R^{\prime}}{\mu \sqrt{1+R^{\prime 2}}} \\
& -\frac{899}{197589} \frac{w_{s} \rho R^{4} g R^{\prime} R^{\prime \prime}}{\mu\left(1+R^{\prime 2}\right)^{3 / 2}}+\frac{5125}{395178} \frac{w_{s} Q \rho^{\prime}}{\rho^{2} \pi} \frac{61}{263452} \frac{R^{5} \rho^{2} g^{2} R^{\prime}}{\mu^{2}}+\frac{61}{790356} \frac{R^{6} \rho g^{2} \frac{d \rho}{d z}}{\mu^{2}} \\
& +\frac{298}{329315} \frac{w_{s}^{2} R R^{\prime 3}}{1+R^{\prime 2}}+\frac{298}{329315} \frac{w_{s}^{2} R^{2} R^{\prime} R^{\prime \prime}}{\left(1+R^{\prime 2}\right)}-\frac{298}{329315} \frac{w_{s}^{2} R^{2} R^{\prime 3} R^{\prime \prime}}{\left(1+R^{\prime 2}\right)^{2}} \\
& +\frac{95}{395178} \frac{w_{s} \frac{d \rho}{d z} R^{4} g}{\mu}+\frac{190}{197589} \frac{w_{s} \rho R^{3} g R^{\prime}}{\mu}+\frac{23837}{987945} \frac{w_{s}^{2} R R^{\prime}}{\sqrt{1+R^{\prime 2}}}-\frac{23837}{1975890} \frac{w_{s}^{2} R^{2} R^{\prime} R^{\prime \prime}}{\left(1+R^{\prime 2}\right)^{3 / 2}} \\
& -\frac{631}{65863} \frac{R Q g R^{\prime}}{\mu \pi}-\frac{13220}{9409} \frac{Q^{2} R^{\prime}}{R^{3} \rho^{2} \pi^{2}}-\frac{13220}{9409} \frac{Q^{2} \frac{d \rho}{d z}}{R^{2} \rho^{3} \pi^{2}}
\end{aligned}
$$

Step 4: At this point everything from Steps 1-3, namely (3.27), (3.28) and (3.29), are substituted into the pressure gradient equation (3.23). Additionally, the resulting equation is divided by $\nu \bar{W}$ so as to write all terms in the equation in dimensionless form. Dimensionless Mach and Reynolds numbers become factors in the equation, being defined as follows:

$$
\begin{gathered}
M a=\frac{\bar{W}}{\sqrt{\frac{k_{B} T}{m}}} \quad \text { (Mach number) } \\
R e=\frac{\rho \bar{W} R}{\mu}=\frac{\bar{W} R}{\nu} \quad \text { (Reynolds number) }
\end{gathered}
$$


Collecting terms and coefficients with respect to $\frac{d^{2} P}{d z^{2}},\left(\frac{d P}{d z}\right)^{2},\left(\frac{d P}{d z}\right)$ and $\left(\frac{d P}{d z}\right)^{0}$ gives:

Coefficient of $\frac{d^{2} P}{d z^{2}}$

$$
\begin{aligned}
& {\left[\frac{2}{3} \frac{M a^{2}}{R e}+\frac{61}{1580712} \frac{R^{2} R e}{\rho} \frac{d P}{d z}-\frac{95}{790356} \frac{w_{s}(R e)}{\bar{W}}-\frac{899}{395178} \frac{w_{s}(R e)}{\sqrt{1+R^{\prime 2} W}}+\frac{631}{263452} R e\right.} \\
& \left.-\frac{61}{1580712} \frac{R^{3} g}{\nu^{2}}\right]\left(\frac{R^{3}}{\mu \bar{W}} \frac{d^{2} P}{d z^{2}}\right)
\end{aligned}
$$

Coefficient of $\frac{d P}{d z}$

$$
\begin{aligned}
& {\left[-\frac{75}{194}+\frac{1}{3} \frac{\nu R^{\prime} w_{s} M a^{2}}{R \bar{W}^{2}}-\frac{95}{197589} \frac{w_{s} R^{\prime} R e}{\bar{W}}-\frac{1798}{197589} \frac{w_{s} R^{\prime} R e}{\bar{W} \sqrt{1+R^{\prime 2}}}+\frac{154937}{79036} \frac{\nu w_{s} M a^{2} R e}{R \bar{W}^{4}\left(1+R^{\prime 2}\right)}\right.} \\
& +\frac{899}{395178} \frac{w_{s} R R^{\prime} R^{\prime \prime} R e}{\bar{W}\left(1+R^{\prime 2}\right)^{3 / 2}}+\frac{631}{131726} R^{\prime} R e-\frac{61}{263452} \frac{R^{2} R^{\prime} g R e}{\nu \bar{W}}+\frac{899}{395178} \frac{w_{s} R g M a^{2} R e}{\bar{W}^{3} \sqrt{1+R^{\prime 2}}} \\
& \left.+\frac{5125}{790356} \frac{\nu w_{s} M a^{2} R e}{R \bar{W}^{2}}+\frac{61}{1580712} \frac{\nu R^{3} g^{2} M a^{2} R e}{\bar{W}^{3}}+\frac{95}{790356} \frac{w_{s} R g M a^{2} R e}{\bar{W}^{3}}-\frac{13220}{9409} M a^{2}\right]\left(\frac{R^{2}}{\mu \bar{W}} \frac{d P}{d z}\right)
\end{aligned}
$$

Coefficient of $\left(\frac{d P}{d z}\right)^{2}$

$$
\left[\frac{4}{3} \frac{M a^{4}}{R e^{2}}+\frac{61}{526904} R^{\prime} R e-\frac{631}{263452} M a^{2}-\frac{61}{1580712} R g \frac{M a^{2}}{\bar{W}^{2}} R e\right]\left(\frac{R^{2}}{\mu \bar{W}} \frac{d P}{d z}\right)^{2}
$$

Coefficient of $\left(\frac{d P}{d z}\right)^{0}$

$$
\begin{aligned}
& {\left[\frac{75}{194} \frac{g R R e}{\bar{W}^{2}}-\frac{R^{\prime \prime} R w_{s}}{\sqrt{1+R^{\prime 2} \bar{W}}}-\frac{R^{\prime 2} w_{s}}{\sqrt{1+\left(R^{\prime}\right)^{2}} \bar{W}}+\frac{2 R^{\prime \prime}\left(R^{\prime}\right)^{2} w_{s} R}{\left(1+R^{\prime 2}\right)^{3 / 2} \bar{W}}-\frac{300}{97}-\frac{12}{97} \frac{w_{s}}{\bar{W}}\right.} \\
& \frac{312}{97} \frac{w_{s}}{\sqrt{1+R^{\prime 2} W}}+\frac{154937}{790356} \frac{\left(R^{\prime}\right)\left(R^{\prime \prime}\right) w_{s} R(R e)}{\left(1+R^{\prime 2}\right)^{3 / 2} \bar{W}}+\frac{26867}{141135} \frac{\left(R^{\prime}\right) w_{s}^{2}(R e)}{\left(1+(R)^{2}\right) \bar{W}^{2}} \\
& -\frac{26867}{141135} \frac{R^{\prime} R^{\prime \prime} w_{s}^{2} R R e}{\left(1+\left(R^{\prime}\right)^{2}\right)^{2} \bar{W}^{2}} \frac{3596}{197589} \frac{R^{3} R^{\prime} g w_{s}}{\nu^{2} \sqrt{1+R^{\prime 2} \bar{W}}}-\frac{899}{395178} \frac{R^{4} R^{\prime} R^{\prime \prime} g w_{s}}{\nu^{2}\left(1+R^{\prime}\right)^{3 / 2} \bar{W}} \\
& +\frac{61}{526904} \frac{(R)^{5}\left(R^{\prime}\right) g^{2}}{\bar{W} \nu^{3}}+\frac{298}{658630} \frac{R R^{\prime} R^{\prime \prime} w_{s}^{2} R e}{\left(1+R^{\prime 2}\right) \bar{W}^{2}} \\
& -\frac{149}{329315} \frac{\left(R^{\prime}\right)^{3} R^{\prime \prime} w_{s}^{2} R R e}{\left(1+\left(R^{\prime}\right)^{2}\right)^{2} \bar{W}^{2}}+\frac{95}{197589} \frac{R^{3} R^{\prime} g w_{s}}{\nu^{2} \bar{W}}+\frac{23837}{1975890} \frac{R^{\prime} w_{s}^{2} R e}{\sqrt{1+\left(R^{\prime}\right)^{2}} \bar{W}^{2}} \\
& \left.-\frac{23837}{3951780} \frac{\left(R^{\prime}\right)\left(R^{\prime \prime}\right) w_{s}^{2} R(R e)}{\left(1+\left(R^{\prime}\right)^{2}\right)^{3 / 2} \bar{W}^{2}}-\frac{631}{131726} \frac{R^{3} R^{\prime} g}{\nu^{2}}-\frac{13220}{(2)(9409)} \frac{R R^{\prime}}{\nu}\right]
\end{aligned}
$$




\subsection{Pressure gradient and Pressure solution}

The pressure gradient equation derived in the previous section is an ODE for $P$ and is in the form

$$
D\left(\frac{R^{3}}{\mu \bar{W}} \frac{d^{2} P}{d z^{2}}\right)+D_{1}\left(\frac{R^{2}}{\mu \bar{W}} \frac{d P}{d z}\right)^{2}+D_{2}\left(\frac{R^{2}}{\mu \bar{W}} \frac{d P}{d z}\right)+D_{3}\left(\frac{R^{2}}{\mu \bar{W}} \frac{d P}{d z}\right)^{0}=0
$$

The term $D\left(\frac{R^{3}}{\mu \bar{W}} \frac{d^{2} P}{d z^{2}}\right)$ will be dropped for simplicity, hence, the equation is reduced to a quadratic equation

$$
D_{1} \bar{\lambda}^{2}+D_{2} \bar{\lambda}+D_{3}=0
$$

where $D 1, D_{2}$ and $D_{3}$ are stated below and $\bar{\lambda}=\frac{R^{2}}{\mu \bar{W}} \frac{d P}{d z}$ has been introduced for simplicity.

Thus

$$
\bar{\lambda}=\frac{R^{2}}{\mu \bar{W}} \frac{d P}{d z}=-\frac{\left[D_{2}+\sqrt{\triangle}\right]}{2 D_{1}},
$$

where $\triangle=D_{2}^{2}-4 D_{1} D_{3}$. After looking at the numerical solution, the positive root of the quadratic equation (3.36) does not lead to physically feasible solutions because the pressure was not continuous in the resulting discontinuous pressure curves, therefore, it is not physically possible to maintain. The pressure gradient and pressure can be directly obtained from integrating (3.36). Note that the DE needs to be solved first, as $D_{1}$, $D_{2}$ and $D_{3}$ depend on $\rho$ through the Reynolds number Re, and $\rho$ depends on $P$ through the equation of state. Thus, the DE is solved first, and then used in $\frac{d P}{d z}$ for plots of the pressure gradient.

Now we state the coefficients for the pressure gradient equation:

$$
\begin{aligned}
& D_{1}=-\frac{4}{3} \frac{M a^{4}}{R e^{2}}+\frac{61}{526904} R^{\prime} R e-\frac{631}{263452} M a^{2}-\frac{61}{1580712} \frac{g R M a^{2} R e}{\bar{W}^{2}} \\
& D_{2}=\frac{75}{194}-\frac{1}{3} \frac{R^{\prime} w_{s}}{\bar{W} \sqrt{1+R^{\prime 2}}} \frac{M a^{2}}{R e}-\frac{190}{395178} \frac{w_{s} R^{\prime} R e}{\bar{W}}-\frac{3596}{395178} \frac{w_{s} R^{\prime} R e}{\bar{W} \sqrt{1+R^{\prime 2}}} \\
& +\frac{899}{395178} \frac{w_{s} R R^{\prime} R^{\prime \prime} R e}{\bar{W}\left(1+R^{\prime 2}\right)^{3 / 2}}+\frac{631}{131726} R^{\prime} R e-\frac{61}{263452} \frac{R g R^{\prime}}{\bar{W}^{2}} R e^{2} \\
& +\frac{154937}{790356} \frac{w_{s} M a^{2}}{\bar{W} \sqrt{1+R^{\prime 2}}}+\frac{899}{395178} \frac{w_{s} R g M a^{2} R e}{\bar{W}^{3} \sqrt{1+R^{\prime 2}}}+\frac{5125}{790356} \frac{w_{s} M a^{2}}{\bar{W}} \\
& +\frac{61}{1580712} \frac{R^{2} g^{2} M a^{2}}{\bar{W}^{4}} R^{2}+\frac{95}{790356} \frac{w_{s} R g M a^{2} R e}{\bar{W}^{3}}-\frac{13220}{18818} M a^{2} \\
& D_{3}=-\frac{75}{194} \frac{g R R e}{\bar{W}^{2}}+\frac{R R^{\prime \prime} w_{s}}{\bar{W} \sqrt{1+R^{\prime 2}}}+\frac{R^{\prime 2} w_{s}}{\bar{W} \sqrt{1+R^{\prime 2}}}-\frac{2 R R^{\prime 2} R^{\prime \prime} w_{s}}{\overline{\bar{W}\left(1+R^{\prime}\right)^{3 / 2}}}
\end{aligned}
$$




$$
\begin{aligned}
& +\frac{300}{97}+\frac{12}{97} \frac{w_{s}}{\bar{W}}-\frac{312}{97} \frac{w_{s}}{\bar{W} \sqrt{1+R^{\prime 2}}}+\frac{154937}{790356} \frac{R R^{\prime} R^{\prime \prime} w_{s} R e}{\bar{W}\left(1+R^{\prime 2}\right)^{3 / 2}} \\
& +\frac{376318}{1975890} \frac{R^{\prime} w_{s}^{2} R e}{\bar{W}^{2}\left(1+R^{\prime 2}\right)}-\frac{376318}{1975890} \frac{R R^{\prime} R^{\prime \prime} R e w_{s}^{2}}{\bar{W}^{2}\left(1+R^{\prime 2}\right)^{2}} \\
& +\frac{3596}{395178} \frac{R R^{\prime} g w_{s}}{\bar{W}^{3} \sqrt{1+R^{\prime 2}}} R e^{2}-\frac{899}{395178} \frac{R^{2} R^{\prime} R^{\prime \prime} g w_{s}}{\bar{W}^{3}\left(1+R^{\prime 2}\right)^{3 / 2}} R e^{2} \\
& +\frac{61}{526904} \frac{R^{2} R^{\prime} g^{2}}{\bar{W}^{4}} R e^{3}+\frac{298}{658630} \frac{R^{\prime 3} w_{s}^{2} R e}{\bar{W}^{2}\left(1+R^{\prime 2}\right)} \\
& +\frac{298}{658630} \frac{R R^{\prime} R^{\prime \prime} w_{s}^{2} R e}{\bar{W}^{2}\left(1+R^{\prime 2}\right)}-\frac{298}{658630} \frac{R R^{\prime 3} R^{\prime \prime} w_{s}^{2} R e}{\bar{W}^{2}\left(1+R^{\prime 2}\right)^{2}} \\
& +\frac{190}{395178} \frac{R R^{\prime} g w_{s}}{\bar{W}^{3}} R e^{2}+\frac{23837}{1975890} \frac{R^{\prime} w_{s}^{2} R e}{\bar{W}^{2}{\sqrt{1+R^{\prime 2}}}^{\prime 2}} \\
& -\frac{23837}{3951780} \frac{R R^{\prime} R^{\prime \prime} w_{s}^{2} R e}{\bar{W}^{2}\left(1+R^{\prime 2}\right)^{3 / 2}}-\frac{631}{131726} \frac{R R^{\prime} g}{\bar{W}^{2}} R e^{2}-\frac{13220}{18818} R^{\prime} R e
\end{aligned}
$$

Note that the coefficient of the second derivative of $P$ was dropped, namely

$$
\begin{aligned}
D= & \frac{2}{3} \frac{M a^{2}}{R e}+\frac{61}{1580712} \frac{R^{2}}{\rho} R e \frac{d P}{d z}-\frac{95}{790356} \frac{w_{s} R e}{\bar{W}}-\frac{899}{395178} \frac{w_{s} R e}{\bar{W} \sqrt{1+R^{\prime 2}}} \\
& +\frac{631}{263452} \operatorname{Re}-\frac{61}{1580712} \frac{g R}{\bar{W}^{2}} R e
\end{aligned}
$$

is set to zero.

In the next chapter, we use Maple to find the numerical solution of (3.36) for analysis in various flow geometries. Note that we used the classical fourth-order Runge-Kutta method (RK4) to find the numerical solution. 


\section{Chapter 4}

\section{Analysis and discussion}

In this Chapter we plot the solution curves derived in the previous chapter in the various axisymmetric flow geometries provided in Section 2.2. For the Gaussian geometry in the first section, we compare the pressure gradient and pressure curves in the no-slip, incompressible limit, and compare with results in the literature. We then discuss how compressibility and slip changes these curves, as well as the flow geometry. Next we assess the pressure gradient, and pressure curves, in the piece-wise cosinusoidal geometry. Finally, in Section 4.3, we assess our pressure gradient and pressure curves in the piece-wise polynomial geometry, and show how they compare with existing pressure curves in the flow geometry and with existing particle-based simulation results.

Note: $R e=R e_{0} \frac{R_{0}}{R}$ and $M a=\frac{M a_{0} \rho_{0} R_{0}{ }^{2}}{\rho R^{2}}$ are used here.

\subsection{Gaussian Geometry}

\subsubsection{Incompressible, no-slip limit}

In Figures 4.1 and Figure 4.2 we plot pressure gradient and pressure curves as a function of the axial coordinate $z$, for zero slip velocity, and $M a_{0}=0.0000001$ at various Reynolds numbers. We pick $\delta=0.1$ and $b=0.25$.

When comparing to the pressure gradient and pressure curves presented in Yao [21], it can be seen that there is good qualitative agreement between our results and theirs in this incompressible, no-slip limit of our solution. Note that the Reynolds numbers in Yao [21] are not the same definition as ours, and relate to our Reynolds number as follows: $R e_{0}^{Y a o}=2 R e_{0}$. In the pressure gradient curves, like Yao [21], we observe an increase in the maxima and a decrease in the minima as the Reynolds number increases. Compared to Yao, the peaks of our pressure gradients are larger than those from Yao, and this difference decreases for the larger Reynolds numbers. For example the peak of the $R e_{0}=200$ curve in our Figure 4.1 is at approximately 200 , while the peak of Yao's $R e_{0}^{Y a o}=400$ curve is at approximately 110 , but the peaks of the $R e_{0}=300$ curves compare as 300 here, and 250 in Yao [21]

In the pressure curves, like Yao [21], we observe the trend that, as Reynolds number increases, the peaks 


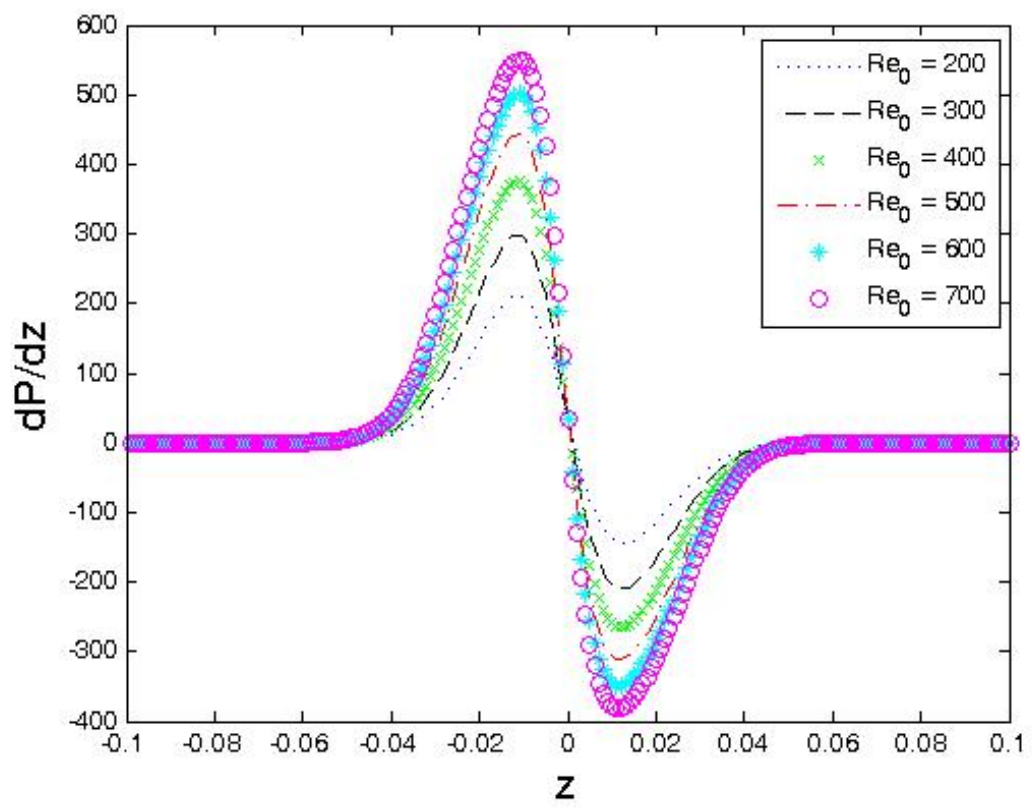

Figure 4.1: Pressure gradient curves in Gaussian geometry with $\delta=0.1, b=0.25, w_{s}=0$, and $M a_{0}=$ 0.00000001 for different Reynolds numbers.

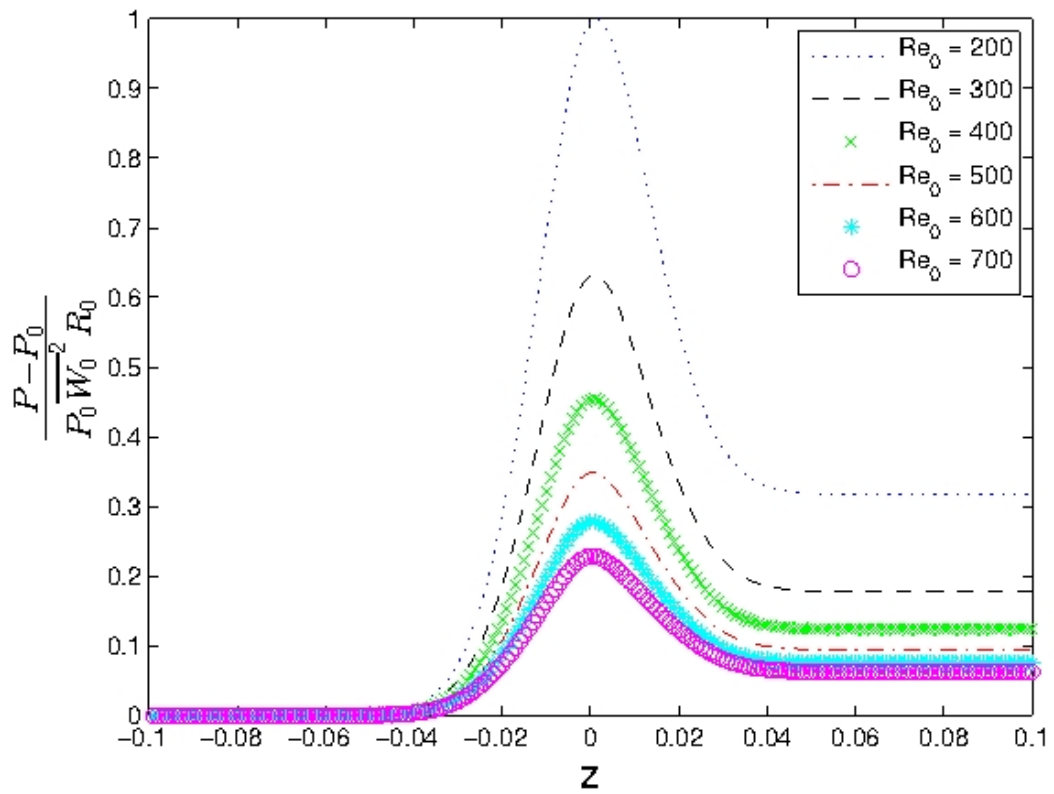

Figure 4.2: Pressure curves in Gaussian geometry with $\delta=0.1, b=0.25, w_{s}=0$, and $M a_{0}=0.00000001$ for different Reynolds numbers. 


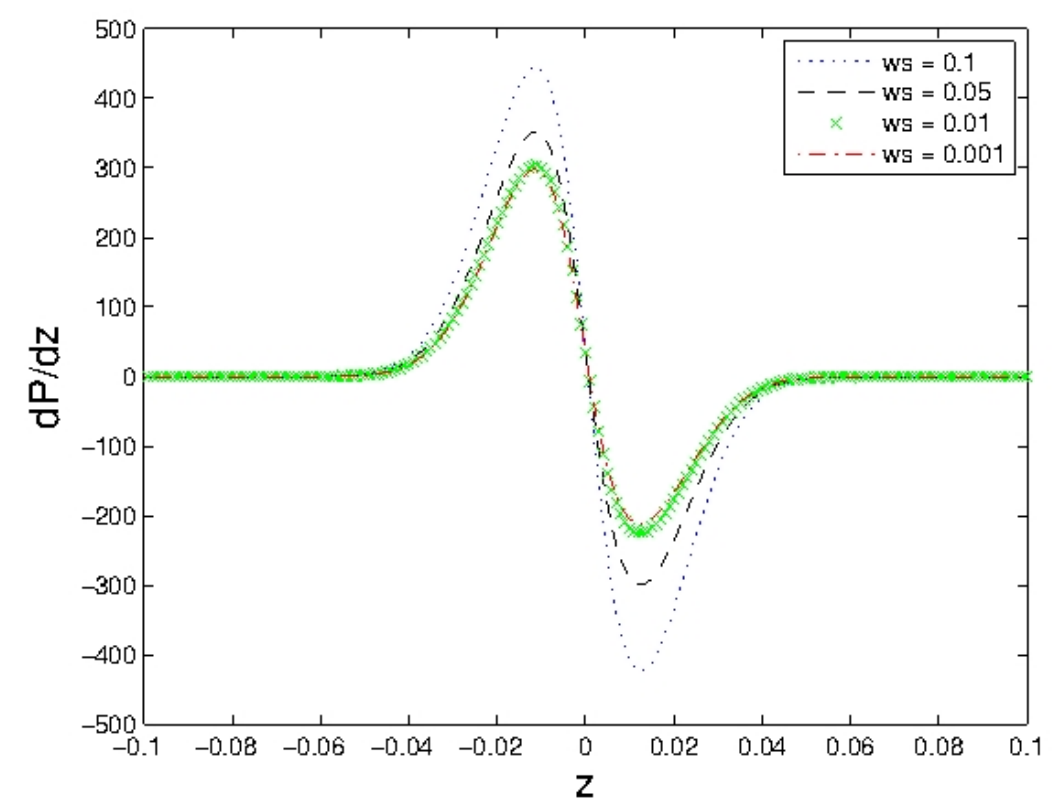

Figure 4.3: Pressure gradient in Gaussian geometry with $\delta=0.1, b=0.25, M a_{0}=0.00000001$ and $R e_{0}=300$ as a function of $z$ for various slip values.

of the pressure curves decrease and reach a value after the constriction that is larger than the pressure before the constriction, and decreases with increasing Reynolds number. Note however one key distinguishing feature in our pressure curves, namely that the pressure after the constriction is always larger than before the constriction, while in Yao's results [21], the pressures after the constriction become negative for larger Reynolds numbers.

\subsubsection{Effect of slip}

For the flow geometry picked in the previous subsection, we plot pressure gradient and pressure curves for $R e_{0}=300$ and consider different values for slip.

In the incompressible limit, we see from Figures 4.3 and 4.4 that the pressure gradient peaks increase with increase in slip. Interestingly enough, the pressure curves reveal that for larger values of slip, the pressure after the constriction becomes lower and eventually negative (not shown), a feature that was missing in Figure 4.2 when compared to Yao's results [21].

For weakly compressible flow $\left(M a_{0}=0.1\right)$, glancing at Figures 4.5 and 4.6 , these trends are maintained, namely, increasing slip increases the peaks in both the pressure and pressure gradient curves, and lowers the limiting value in the pressure curves after the constriction. 


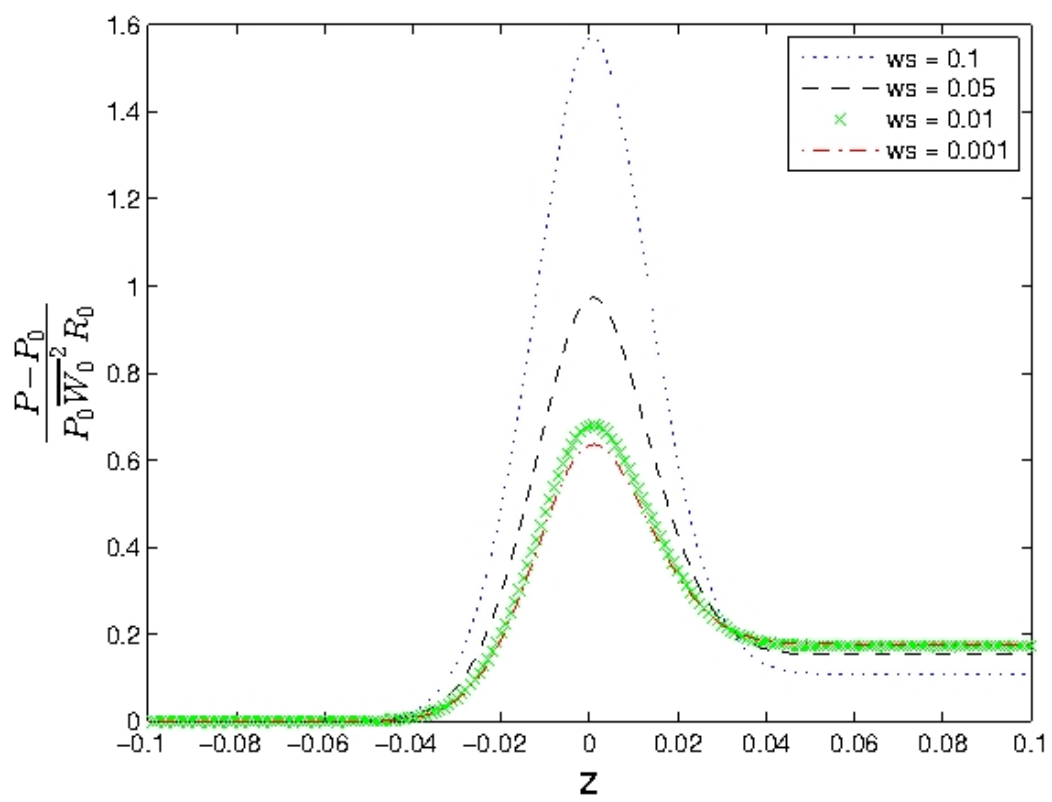

Figure 4.4: Pressure curves in Gaussian geometry with $\delta=0.1, b=0.25, M a_{0}=0.00000001$ and $R e_{0}=300$ as a function of $z$ for various slip values.

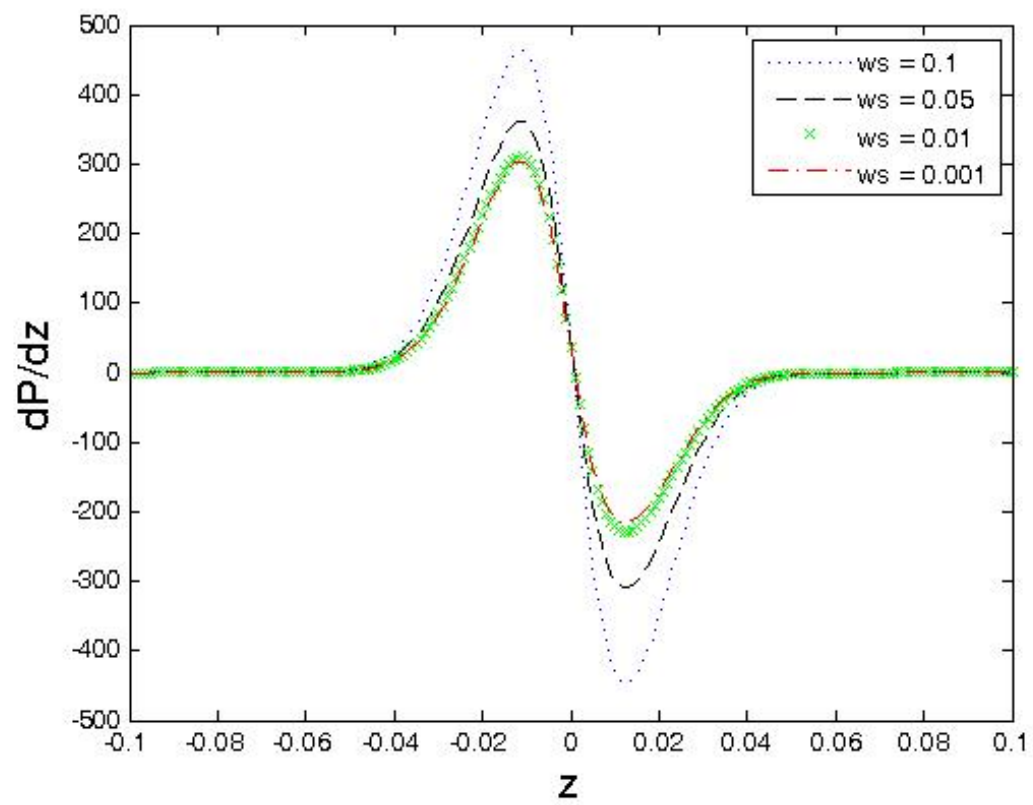

Figure 4.5: Pressure gradient in Gaussian geometry with $\delta=0.1, b=0.25, M a_{0}=0.1$ and $R e_{0}=300$ as a function of $z$ for various slip values. 


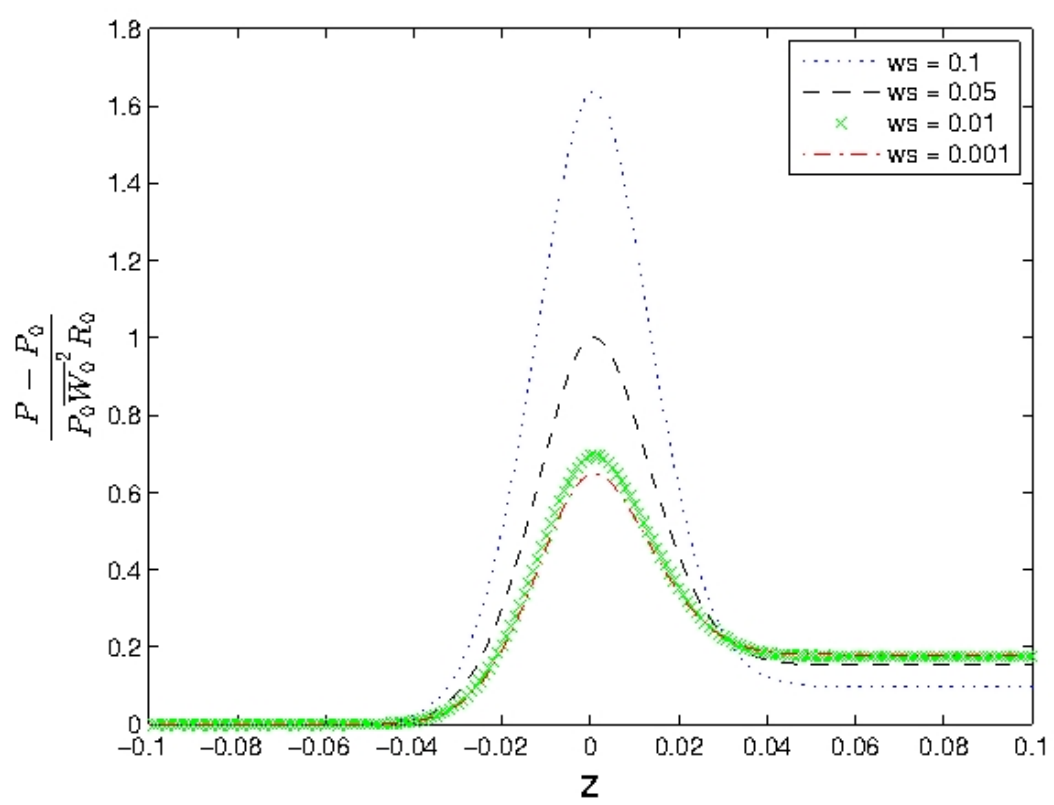

Figure 4.6: Pressure curves in Gaussian geometry with $\delta=0.1, b=0.25, M a_{0}=0.1$ and $R e_{0}=300$ as a function of $z$ for various slip values.

\subsubsection{Effect of compressibility}

In order to study the effect of varying $M a_{0}$ number, we pick various $M a_{0}$ numbers for same Reynolds number and constriction ratio as considered in the previous subsection. We now plot pressure gradient and pressure curves for different $M a_{0}$ numbers. In Figures 4.7 and 4.8 , we consider the no-slip case $w_{s}=0$, and in Figures 4.9 and 4.10, we consider a slip factor $w_{s}=0.1$. In this study, by comparing the pressure gradient and pressure curves for $M a_{0}=(0.001,0.05,0.15,0.2)$, we can find that the flow patterns are very similar in the case of no slip $\left(w_{s}=0\right)$, in fact there is a noticeable overlap. However, when setting $M a_{0}=0.5$ we observe an obvious difference in the maxima and minima of the pressure and pressure gradient curves. Furthermore, the higher the $M a_{0}$ number is the larger the pressure gradient and pressure curves, in addition, we obtain larger positive pressure curves after the constriction, which is again a key feature. It is worth noting here that in the case of slip $\left(w_{s}=0.1\right)$, the pressure gradient curves increase as we increase $M a_{0}$ number. At the same time, the pressure curves increase as we increase $M a_{0}$ number. An important feature occurs here when we increase $M a_{0}$ number, the pressure curve decreases and becomes negative after the constriction.

Although the pressure gradient and pressure curves have the same pattern in the case of no slip $\left(w_{s}=0\right)$ and slip $\left(w_{s}=0.1\right)$, we notice that there is less of an overlap when encountering the case of slip $\left(w_{s}=0.1\right)$. It can be concluded that $M a_{0}$ number has evident effects on the flow. The larger $M a_{0}$ number will make the curves more complex and cause variables to vary in a greater range especially in the case of slip $\left(w_{s}=0.1\right)$. 


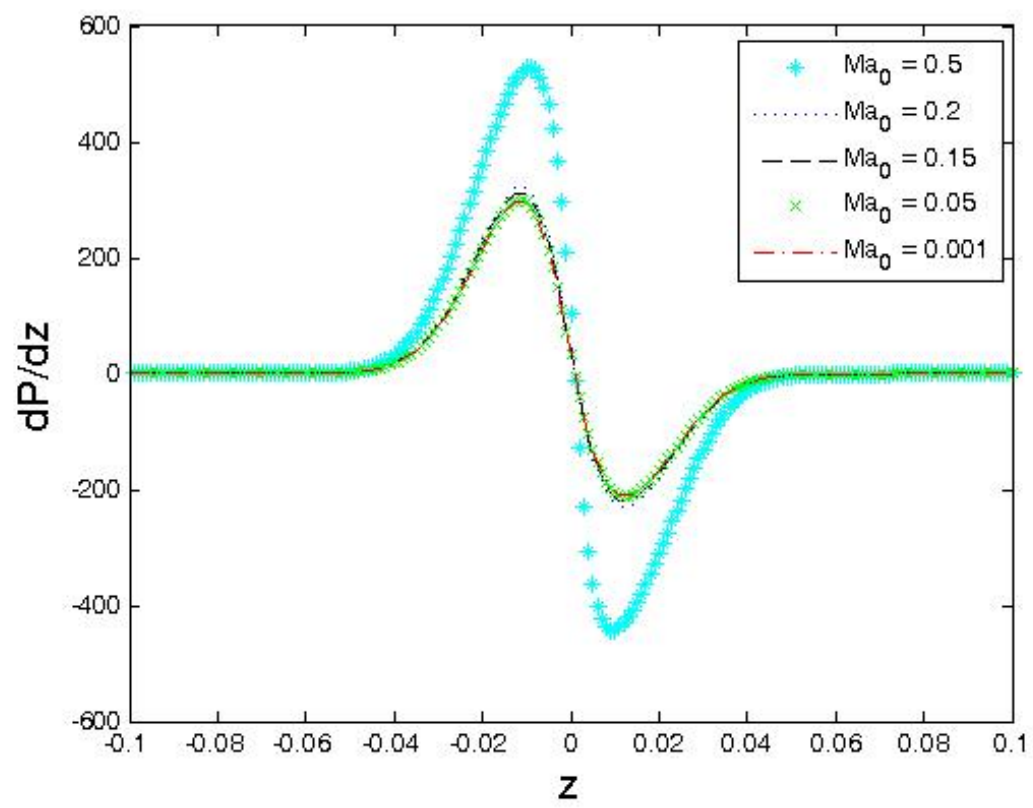

Figure 4.7: Pressure gradient in Gaussian geometry with $\delta=0.1, b=0.25, w_{s}=0$ and $R e_{0}=300$ as a function of $z$ for various $M a_{0}$ numbers.

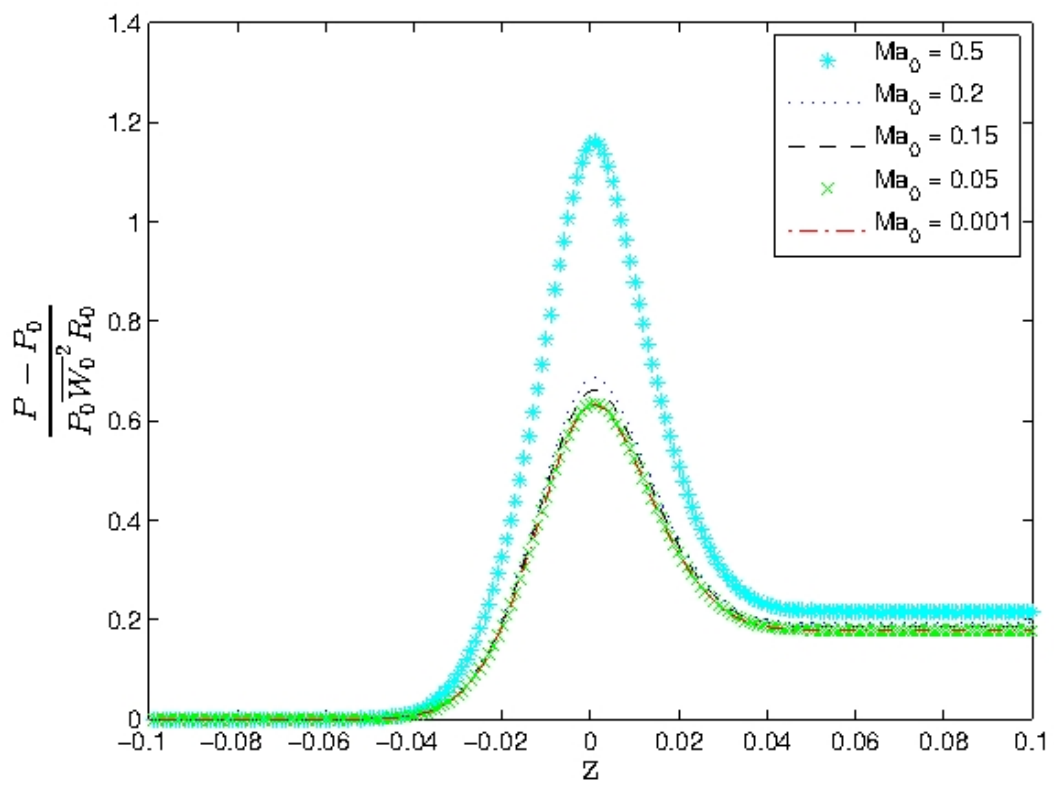

Figure 4.8: Pressure curves in Gaussian geometry with $\delta=0.1, b=0.25, w_{s}=0$ and $R e_{0}=300$ as a function of $z$ for various $M a_{0}$ numbers. 


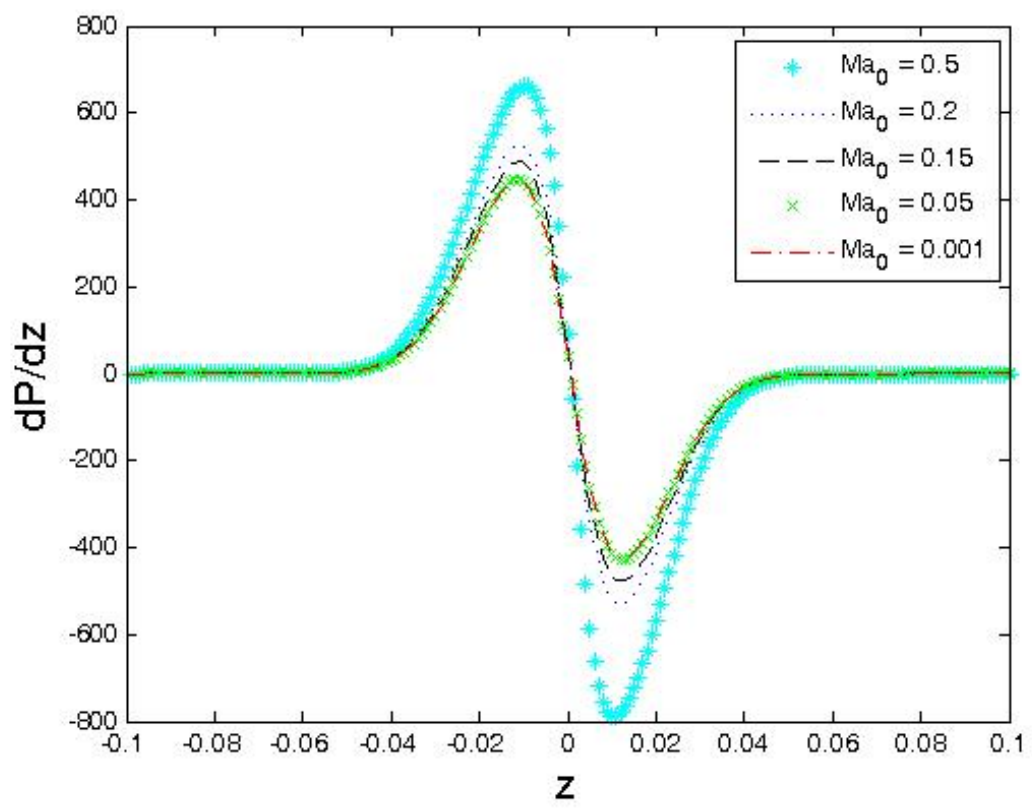

Figure 4.9: Pressure gradient curves in Gaussian geometry with $\delta=0.1, b=0.25, w_{s}=0.1$ and $R e_{0}=300$ as a function of $z$ for various $M a_{0}$ numbers.

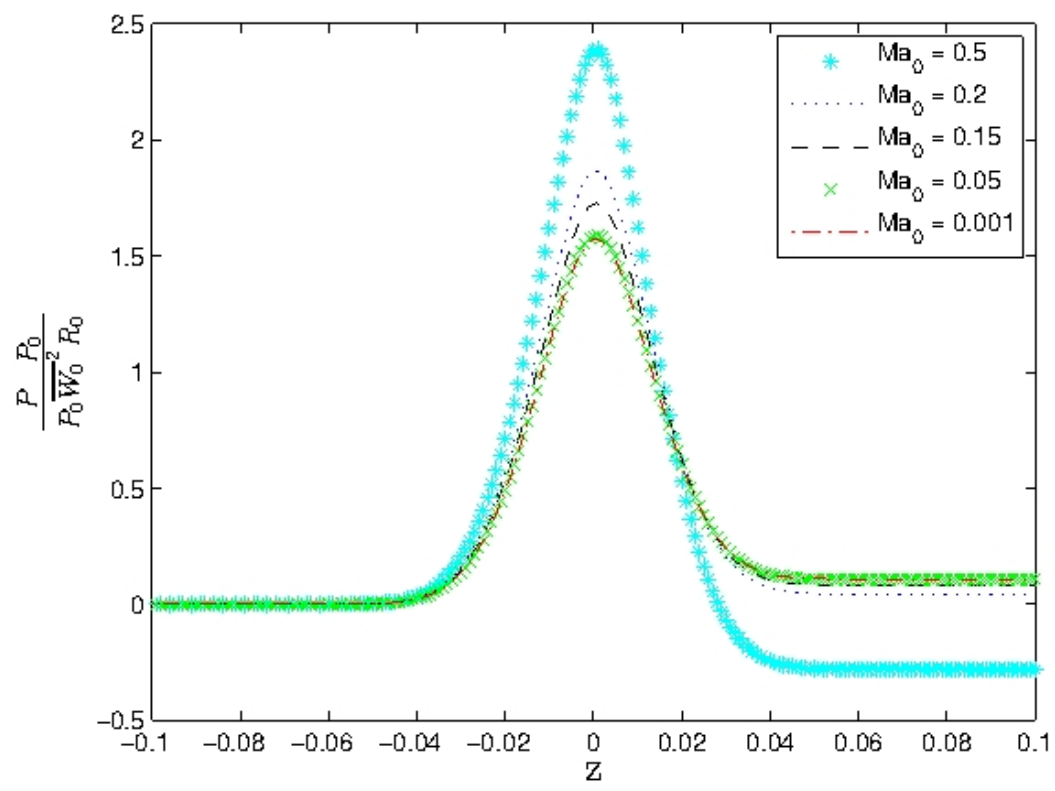

Figure 4.10: Pressure curves in Gaussian geometry with $\delta=0.1, b=0.25, w_{s}=0.1$ and $R e_{0}=300$ as a function of $z$ for various $M a_{0}$ numbers. 


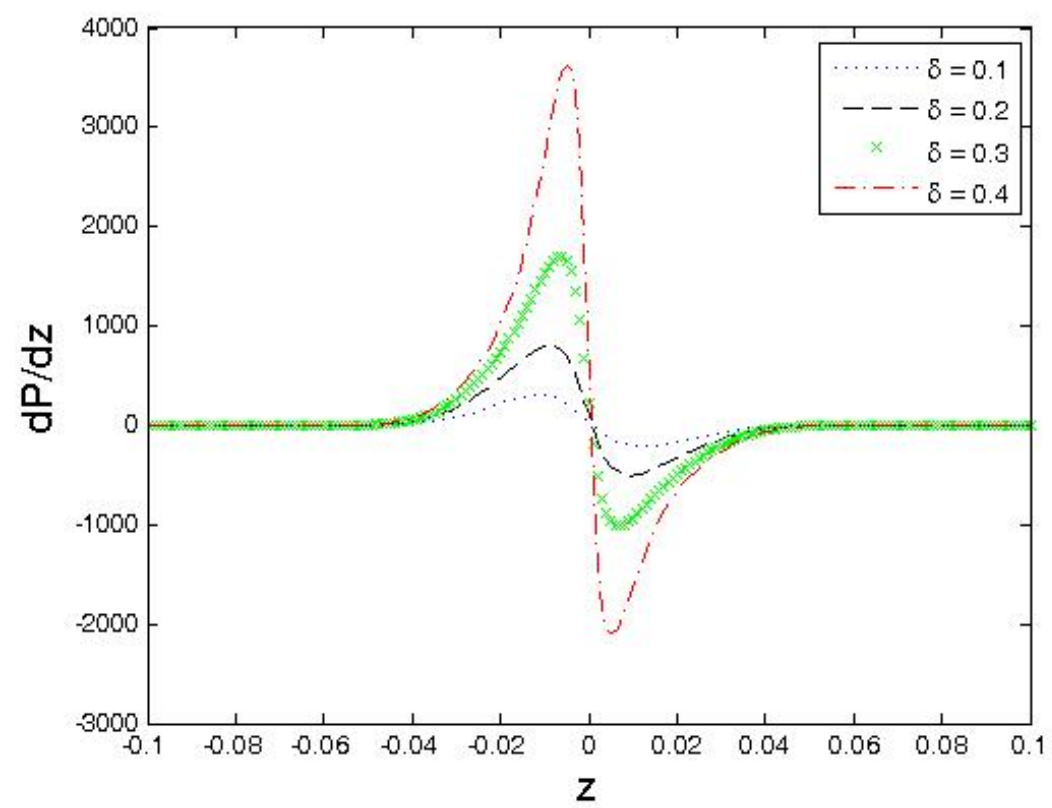

Figure 4.11: Pressure gradient curves in Gaussian geometry with $b=0.25, w_{s}=0, M a_{0}=0.1$ and $R e_{0}=300$ as a function of $z$ for various $\delta$ values.

\subsubsection{Effect of severity of constriction}

It is understood that the constriction ratio, $\delta$, has a great influence on the flow field through the constricted cylinder for steady flow. In our study, we show how the characteristics of the flow vary with the variation of constriction ratios. Constriction ratios $\delta=0.1, \delta=0.2, \delta=0.3$ and $\delta=0.4$ are considered here. Maintaining the same structure and using the flow geometry picked in the previous subsection, we plot pressure gradient and pressure curves for $R e_{0}=300$ and consider different values for $\delta$.

By studying the Figures 4.11 and 4.12 , for no-slip $\left(w_{s}=0\right)$ and weakly compressible flow $\left(M a_{0}=0.1\right)$, and for slip $\left(w_{s}=0.1\right)$ and weakly compressible flow $\left(M a_{0}=0.1\right)$, glancing at Figures 4.13 and 4.14 , the flow developments for the constriction ratio $\delta=0.4$ has a significant differences from that for $\delta=0.1,0.2,0.3$. We notice an increase of the pressure gradient and pressure curves in the case of no-slip and slip. It can be found that the pressure gradient and pressure curves have a very similar pattern, however with increasing constriction ratio to $\delta=0.4$ the curves become larger.

According to the above analysis, the constriction ratio contributes to a distinct effect on the magnitude of pressure gradient and pressure curves. In general, the severe constriction can lead the pressure to increase dramatically. 


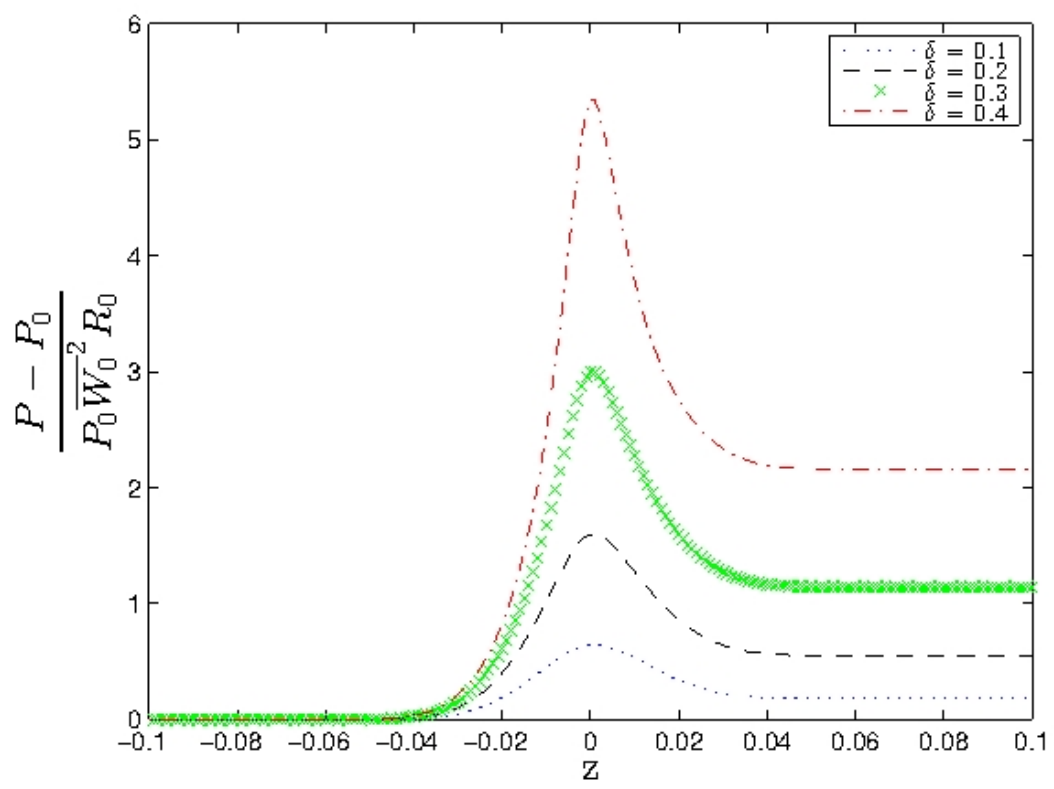

Figure 4.12: Pressure curves in Gaussian geometry with $b=0.25, w_{s}=0, M a_{0}=0.1$ and $R e_{0}=300$ as a function of $z$ for various $\delta$ values

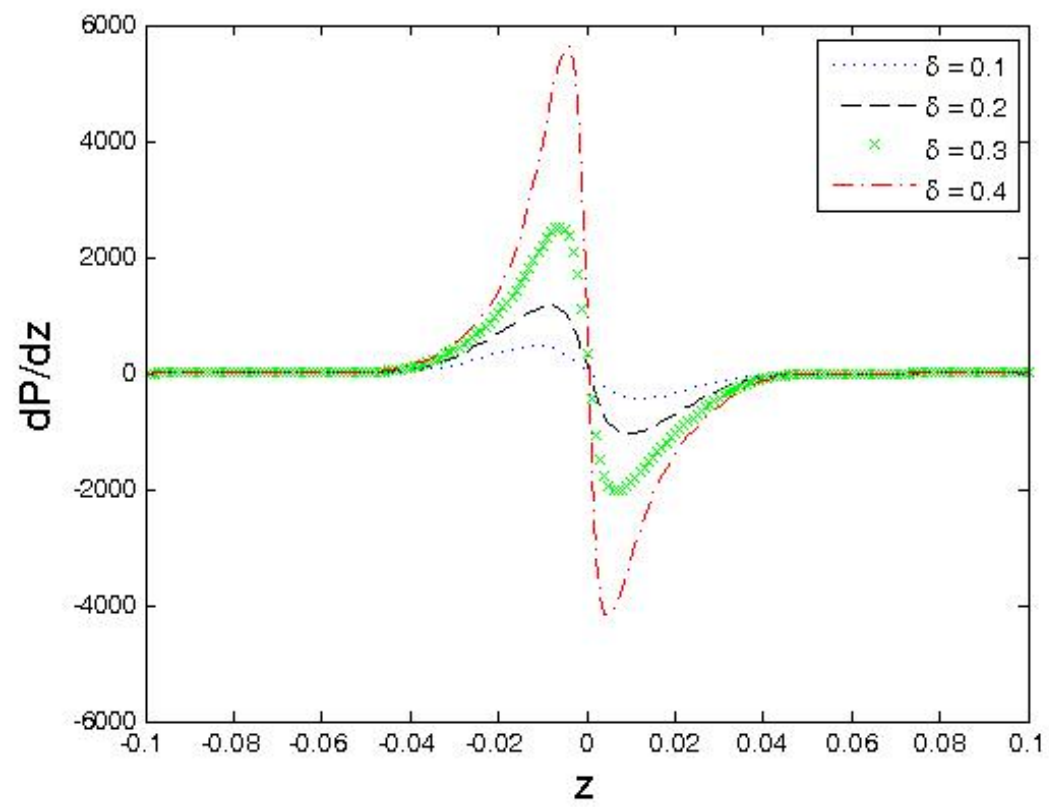

Figure 4.13: Pressure gradient curves in Gaussian geometry with $b=0.25, w_{s}=0.1, M a_{0}=0.1$ and $R e_{0}=300$ as a function of $z$ for various $\delta$ values. 


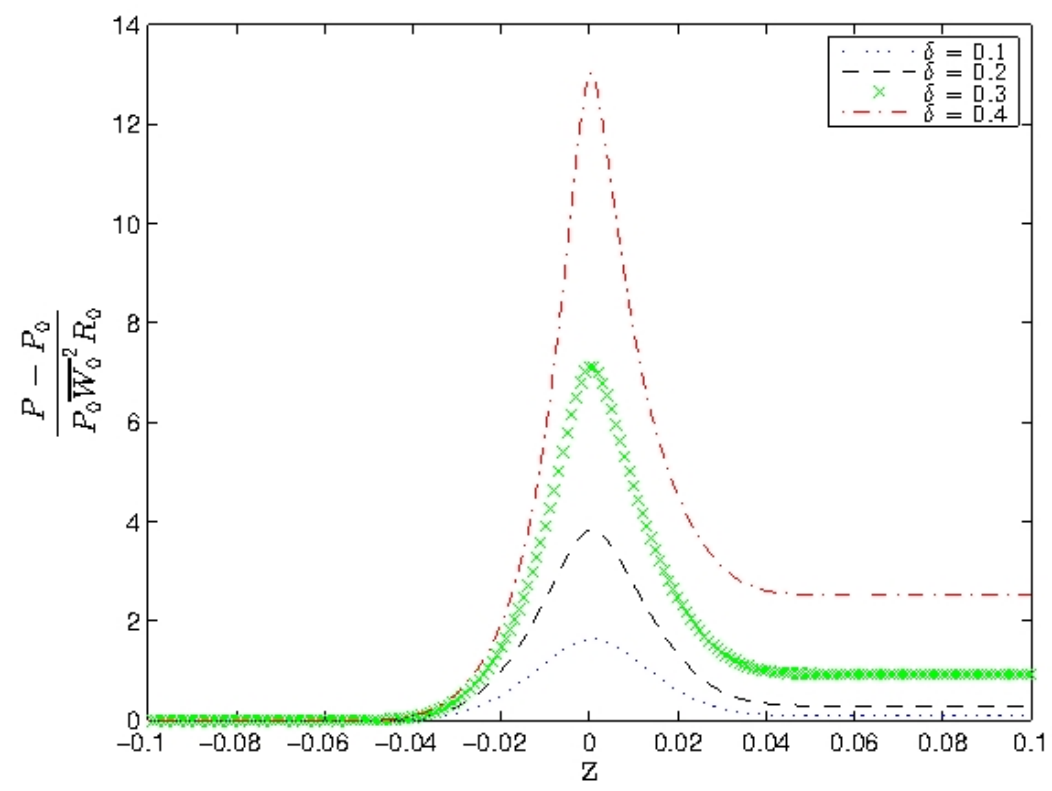

Figure 4.14: Pressure curves in Gaussian geometry with $b=0.25, w_{s}=0.1, M a_{0}=0.1$ and $R e_{0}=300$ as a function of $z$ for various $\delta$ values

\subsubsection{Effect of length of constriction}

Working with the same structure and using the flow geometry picked in the previous subsection, we plot pressure gradient and pressure curves for $R e_{0}=300$ and consider different values for $b$.

In the no-slip $\left(w_{s}=0\right)$ and weakly compressible limit $\left(M a_{0}=0.1\right)$, we see from Figures 4.15 and 4.16 the pressure gradient peaks increase with increase in $b$. However, the pressure curves decrease with an increase in $b$.

For weakly compressible flow $\left(M a_{0}=0.1\right)$ and slip $\left(w_{s}=0.1\right)$, glancing at Figures 4.17 and 4.18 , these trends are maintained, namely, increasing pressure gradient curves as $b$ increases, and the pressure curves decrease as $b$ increases. One main difference here is that by comparing the pressure curves between the slip and no-slip cases, we notice that the pressure after the constriction drops lower in the case of slip $\left(w_{s}=0.1\right)$.

\subsection{Piece-wise Cosinusoidal Geometry}

\subsubsection{No Slip}

In this section, we implement the Cosinusoidal Geometry, and we plot pressure gradient and pressure curves for various $\delta$ and consider different values for $R_{0}$ numbers.

In the no-slip $\left(w_{s}=0\right)$, incompressible limit $\left(M a_{0}=0.0000001\right)$ and for $\delta=0.1$ in Figures 4.19 and 4.20, for $\delta=0.2$ in Figures 4.21 and 4.22 and for $\delta=0.3$ in Figures 4.23 and 4.24, we see that the pressure 


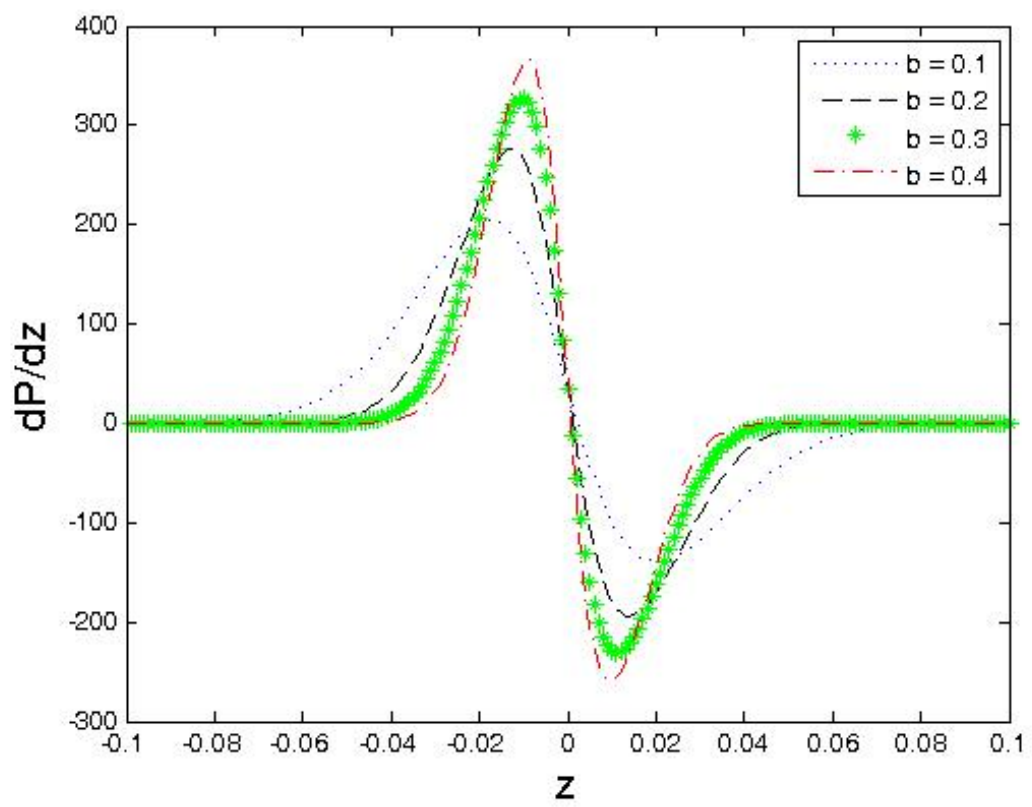

Figure 4.15: Pressure gradient curves in Gaussian geometry with $\delta=0.1, w_{s}=0, M a_{0}=0.1$ and $R e_{0}=300$ as a function of $z$ for various $b$ values.

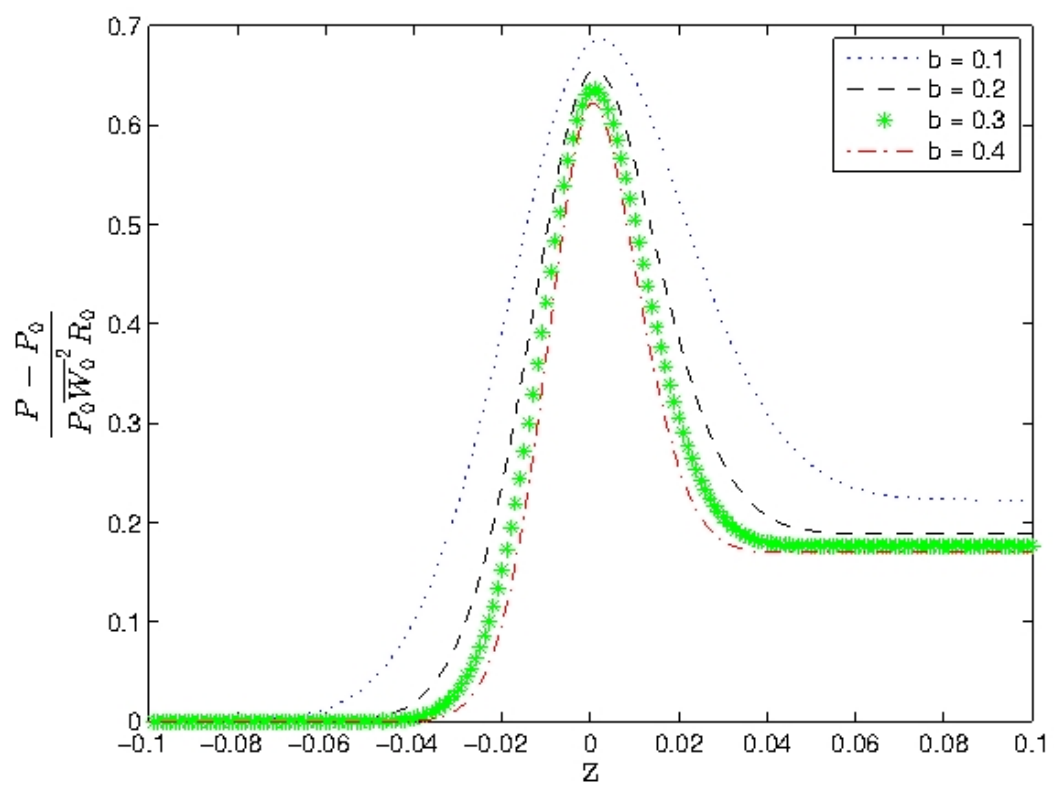

Figure 4.16: Pressure curves in Gaussian geometry with $\delta=0.1, w_{s}=0, M a_{0}=0.1$ and $R e_{0}=300$ as a function of $z$ for various $b$ values 


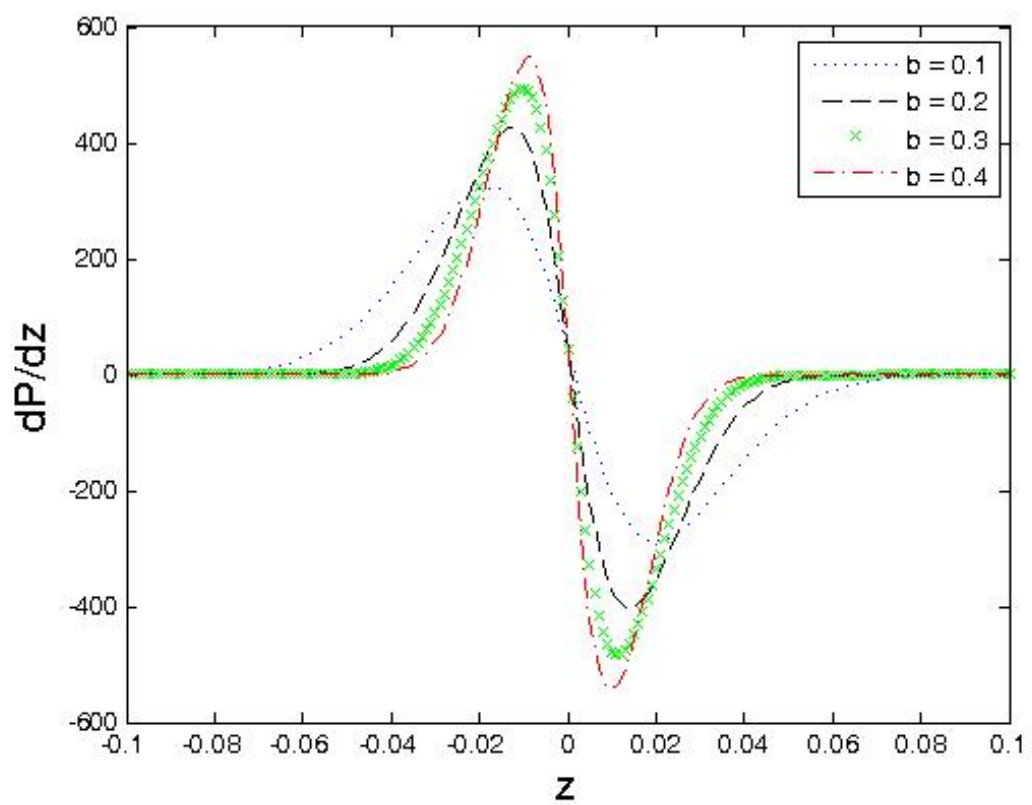

Figure 4.17: Pressure gradient curves in Gaussian geometry with $\delta=0.1, w_{s}=0.1, M a_{0}=0.1$ and $R e_{0}=300$ as a function of $z$ for various $b$ values.

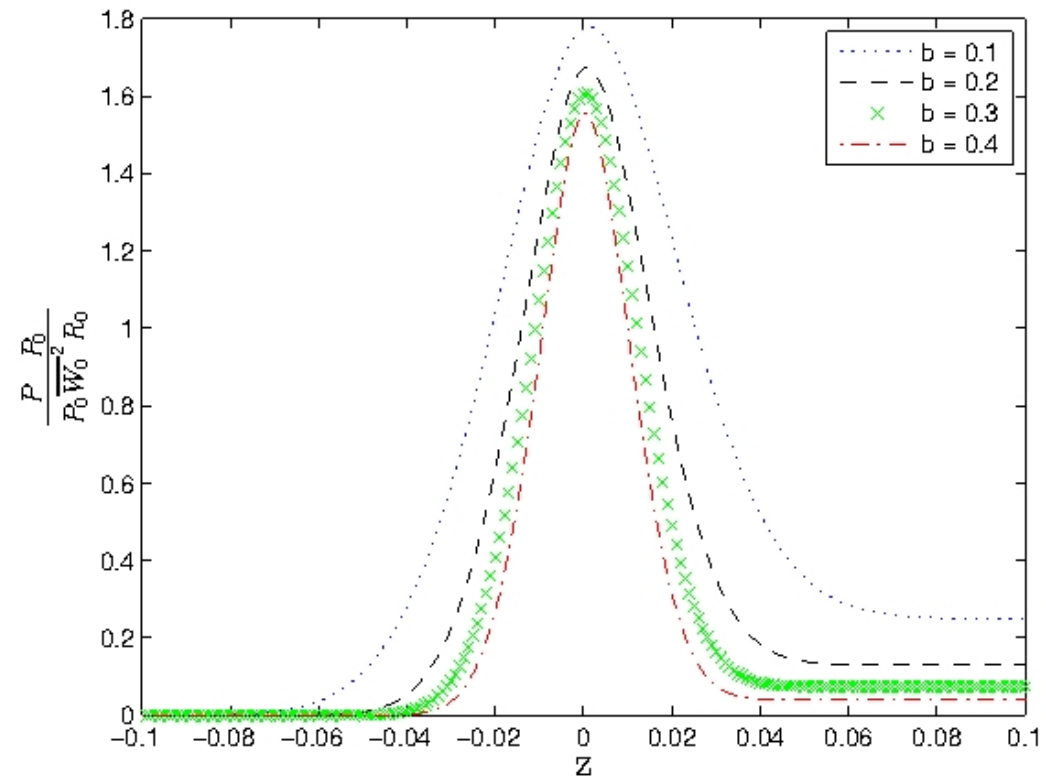

Figure 4.18: Pressure curves in Gaussian geometry with $\delta=0.1, w_{s}=0.1, M a_{0}=0.1$ and $R e_{0}=300$ as a function of $z$ for various $b$ values 


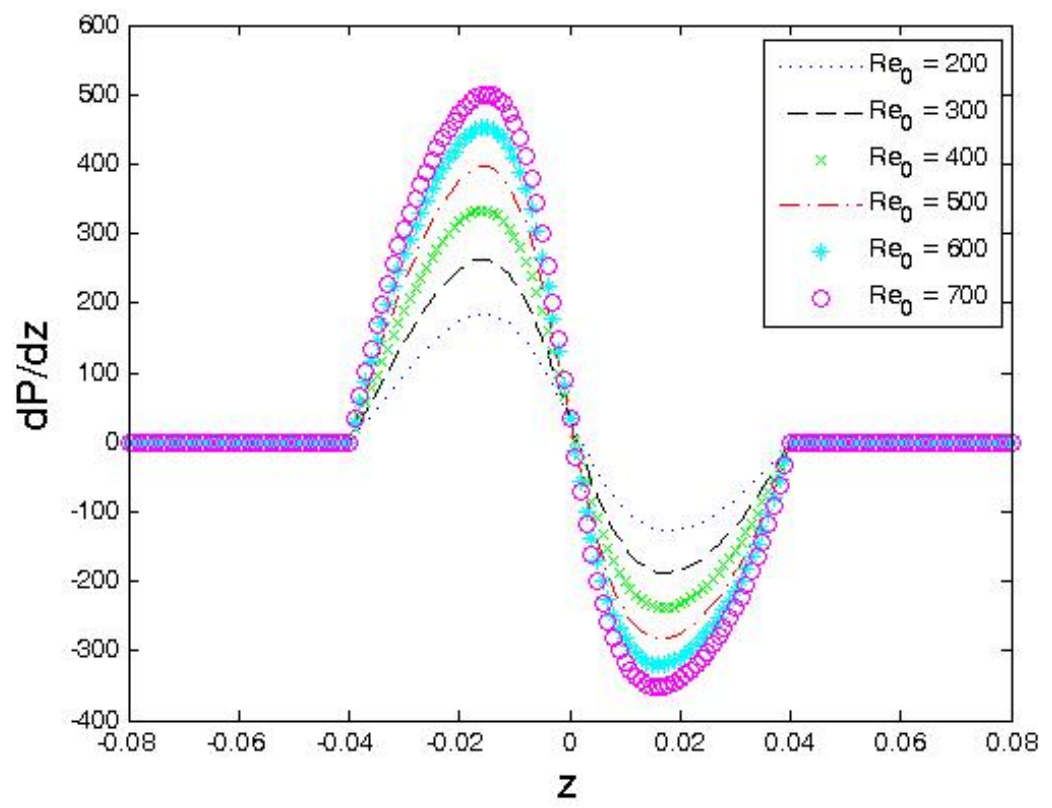

Figure 4.19: Pressure gradient curves in Piece-wise Cosinusoidal Geometry with $\delta=0.1, w_{s}=0$ and $M a_{0}=0.0000001$ as a function of $z$ for various Reynolds numbers.

gradient peaks increase are $R e_{0}$ increases, and we observe an increase in the maxima and a decrease in the minima as the Reynolds number increases. Compared to the Gaussian geometry with $\delta=0.1$, the peaks of the pressure gradients are less than those from Gaussian geometry. For example the peak of the $R e_{0}=700$ curve in Figure 4.19 is at approximately 500, while the peak of Gaussian geomtetry is at $R e_{0}=700$ curve in Figure 4.1 is at approximately 550.

In the pressure curves we observe that, as Reynolds number increases, the peaks of the pressure curves decrease and reach a value after the constriction that is larger than the pressure before the constriction, and decreases with increasing Reynolds number. Note however one key distinguishing feature in the Cosinusoidal geometry pressure curves, namely that the pressure after the constriction is always larger than before the constriction. Compared to the Gaussian geometry with $\delta=0.1$, the peaks of the pressure curves are less than those from Gaussian geometry. For example the peak of the $R e_{0}=700$ curve in Figure 4.20 is slightly higher than 1, while the peak of Gaussian geomtetry is at $R e_{0}=700$ curve in Figure 4.2 is slightly less than 1.

\subsubsection{Slip}

Maintaining the same structure and using the flow geometry picked in the previous subsection, we plot pressure gradient and pressure for $\delta=0.3$ and consider different values for $R_{0}$.

In the slip $\left(w_{s}=0.1\right)$, incompressible limit $\left(M a_{0}=0.0000001\right)$ and $\delta=0.3$, in Figures 4.25 and 4.26 , 


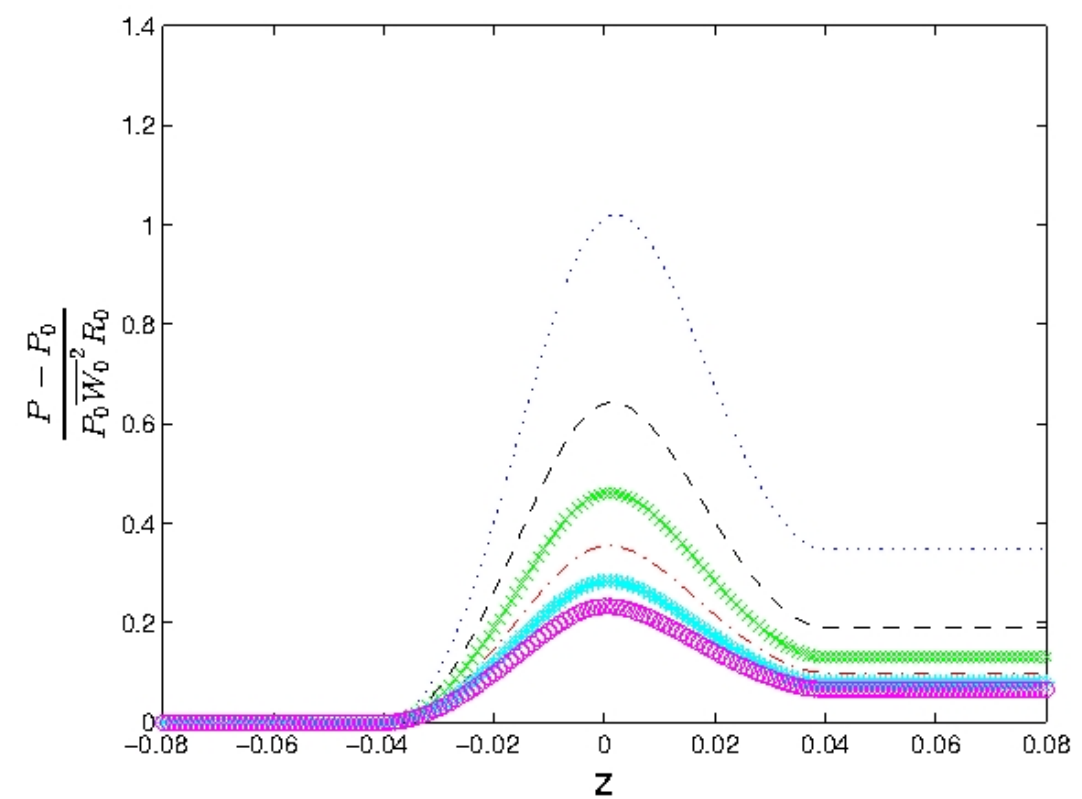

Figure 4.20: Pressure curves in Piece-wise Cosinusoidal Geometry with $\delta=0.1, w_{s}=0$ and $M a_{0}=0.0000001$ as a function of $z$ for various Reynolds numbers.

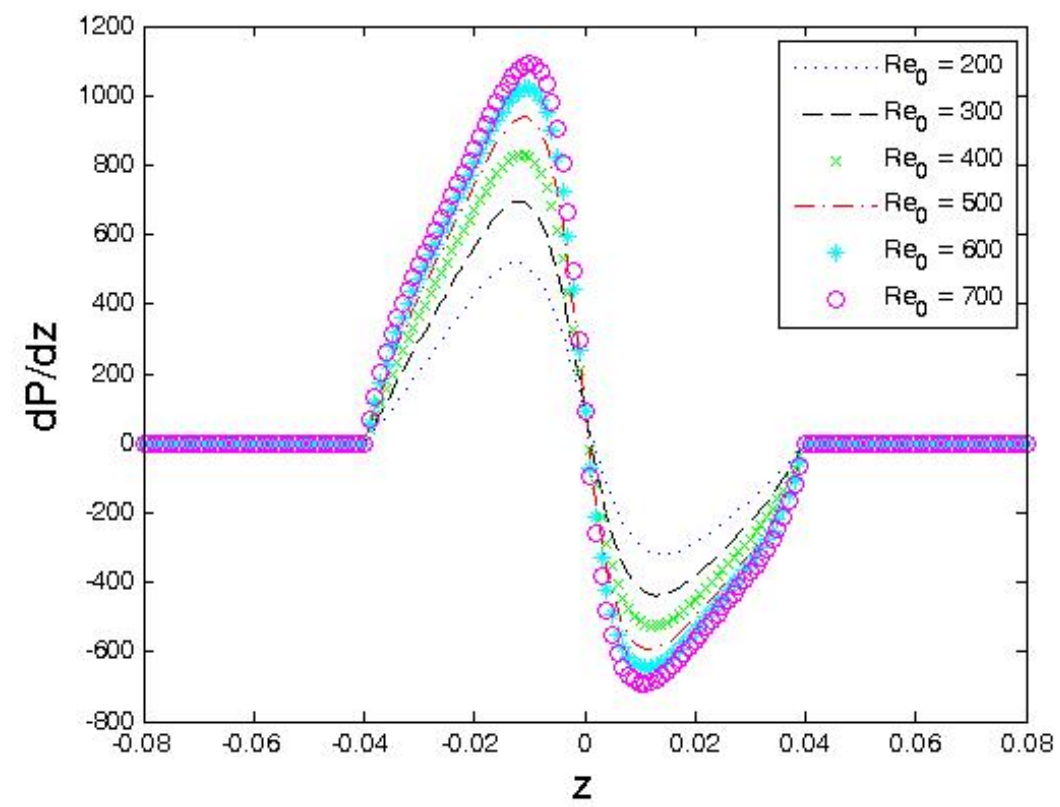

Figure 4.21: Pressure gradient curves in Piece-wise Cosinusoidal Geometry with $\delta=0.2, w_{s}=0$ and $M a_{0}=0.0000001$ as a function of $z$ for various Reynolds numbers. 


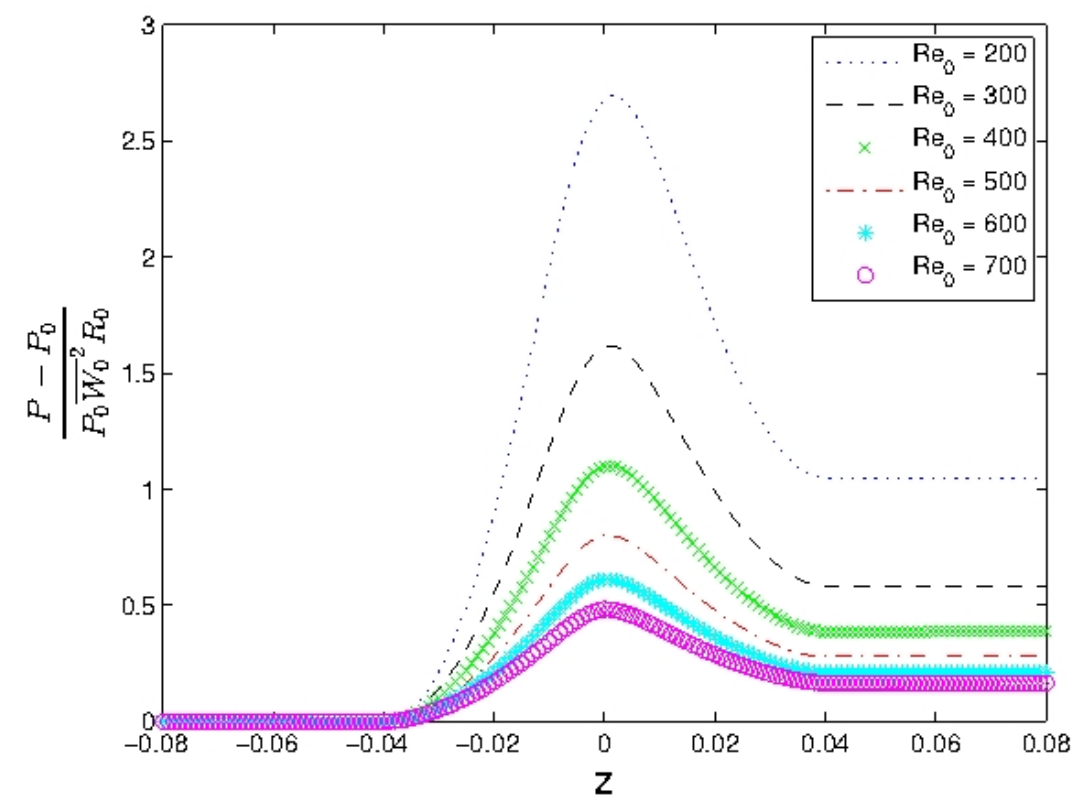

Figure 4.22: Pressure curves in Piece-wise Cosinusoidal Geometry with $\delta=0.2, w_{s}=0$ and $M a_{0}=0.0000001$ as a function of $z$ for various Reynolds numbers.

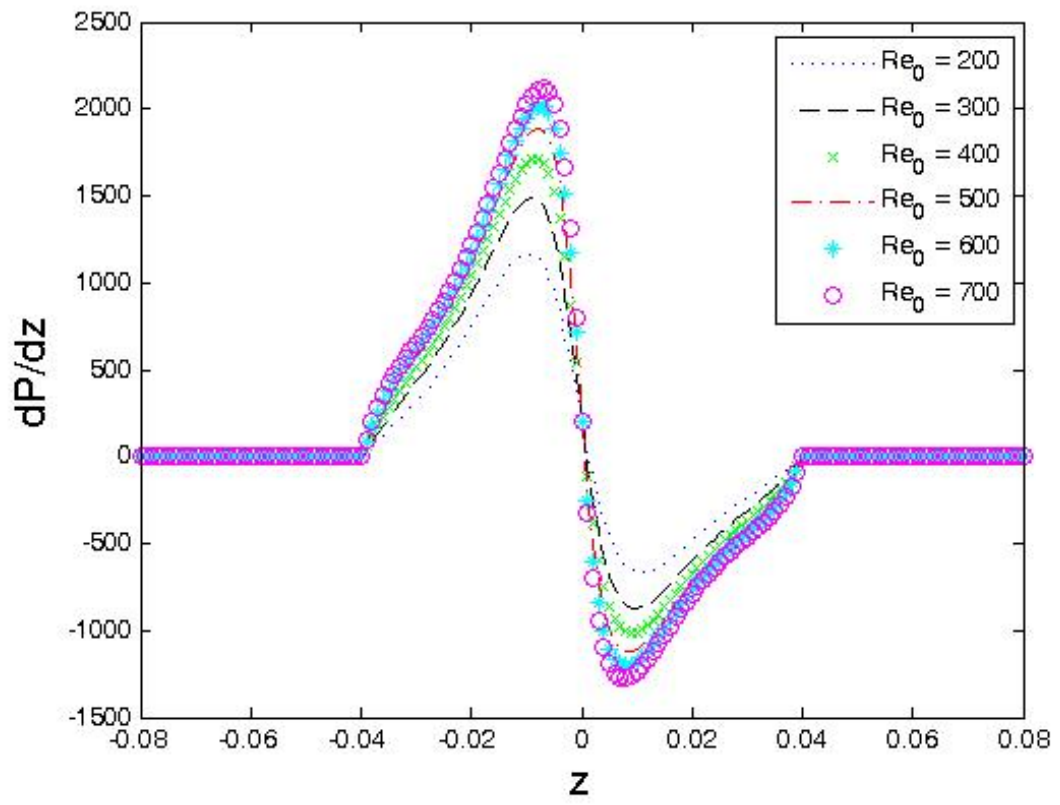

Figure 4.23: Pressure gradient curves in Piece-wise Cosinusoidal Geometry with $\delta=0.3, w_{s}=0$ and $M a_{0}=0.0000001$ as a function of $z$ for various Reynolds numbers. 


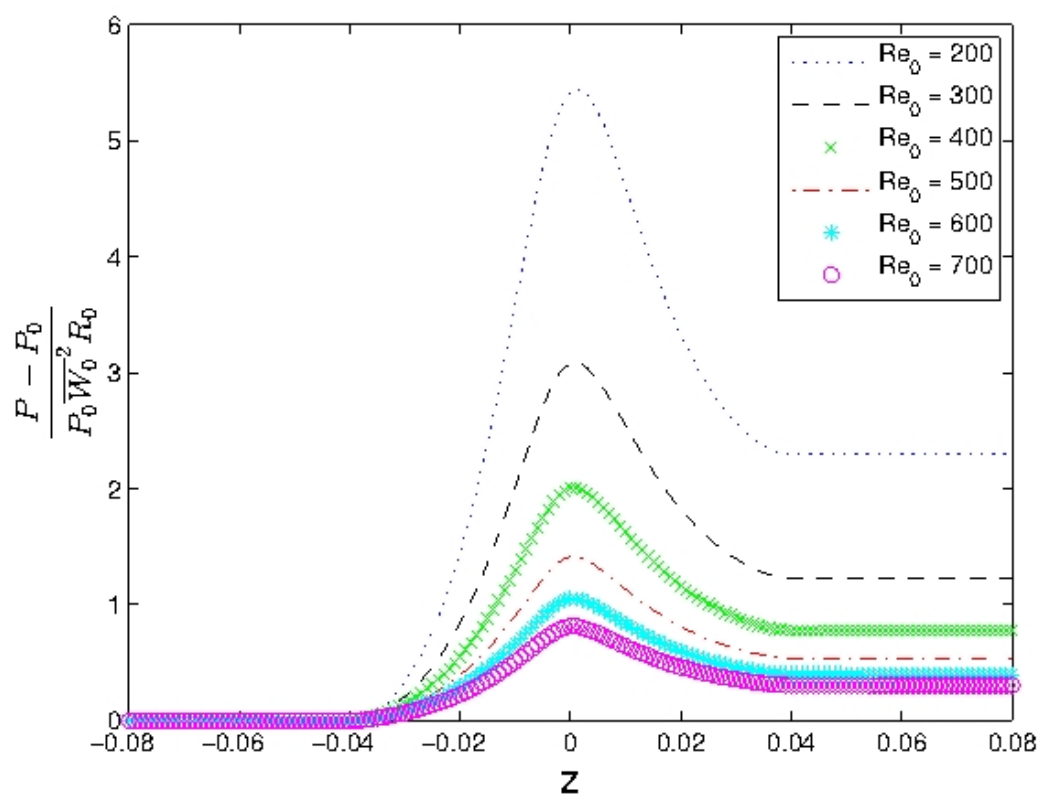

Figure 4.24: Pressure curves in Piece-wise Cosinusoidal Geometry with $\delta=0.3, w_{s}=0$ and $M a_{0}=0.0000001$ as a function of $z$ for various Reynolds numbers.

and we compare that to the case of no-slip $\left(w_{s}=0\right)$ in Figures 4.23 . We observe that the pressure gradient curves have more of an overlap in the slip case comparing to no-slip. Furthermore, the pressure gradient curves in the slip case have a higher magnitude. The same argument can be made where pressure curves are much higher when encountering slip. An additional observation can be made, namely that with slip case, the pressure curves are less in magnitude after the constriction in comparison to the case with no-slip.

For the slip $\left(w_{s}=0.1\right)$, weakly compressible flow $\left(M a_{0}=0.1\right)$ and $\delta=0.3$, glancing at Figures 4.27 and 4.28 , and comparing it with the case of slip $w_{s}=0.1$ and weakly incompressible flow as in Figures 4.25 and 4.26. We notice that in $M a_{0}=0.1$ the pressure gradient curves overlap more and have a larger magnitude than they in the case of $M a_{0}=0.0000001$. Moreover, the pressure curves in $M a_{0}=0.1$ have a higher magnitude than in the case of $M a_{0}=0.0000001$. 


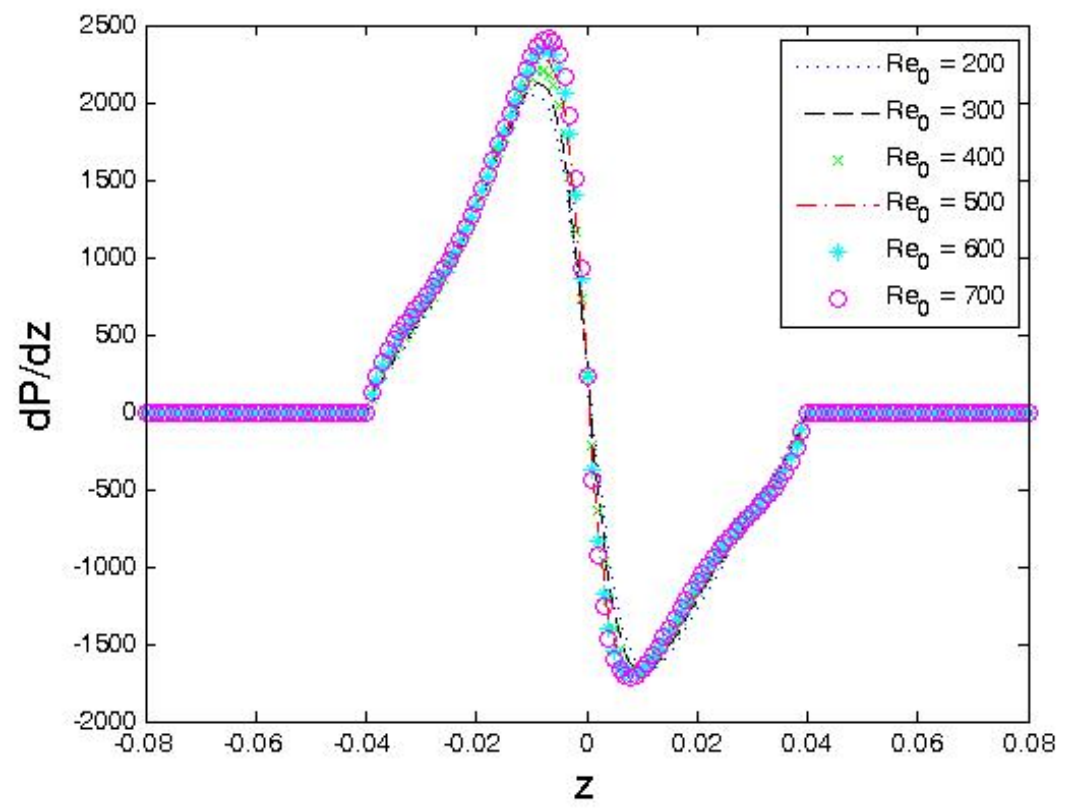

Figure 4.25: Pressure gradient curves in Piece-wise Cosinusoidal Geometry with $\delta=0.3, w_{s}=0.1$ and $M a_{0}=0.0000001$ as a function of $z$ for various Reynolds numbers.

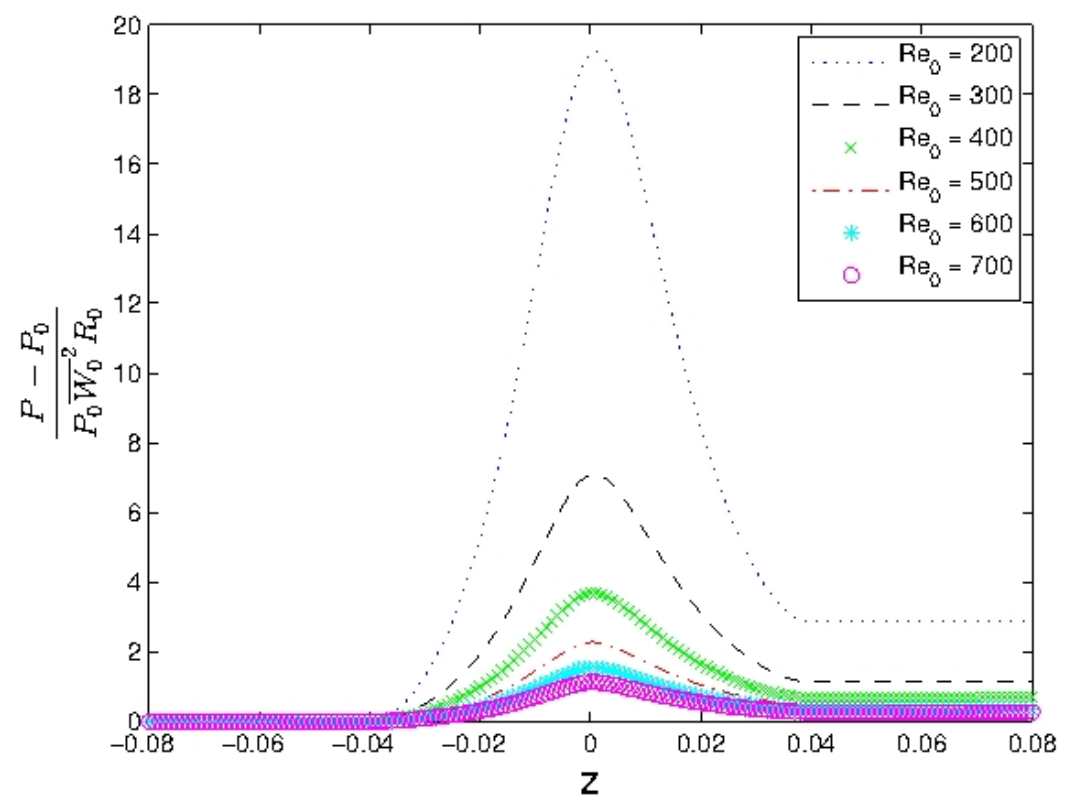

Figure 4.26: Pressure curves in Piece-wise Cosinusoidal Geometry with $\delta=0.3, w_{s}=0.1$ and $M a_{0}=$ 0.0000001 as a function of $z$ for various Reynolds numbers. 


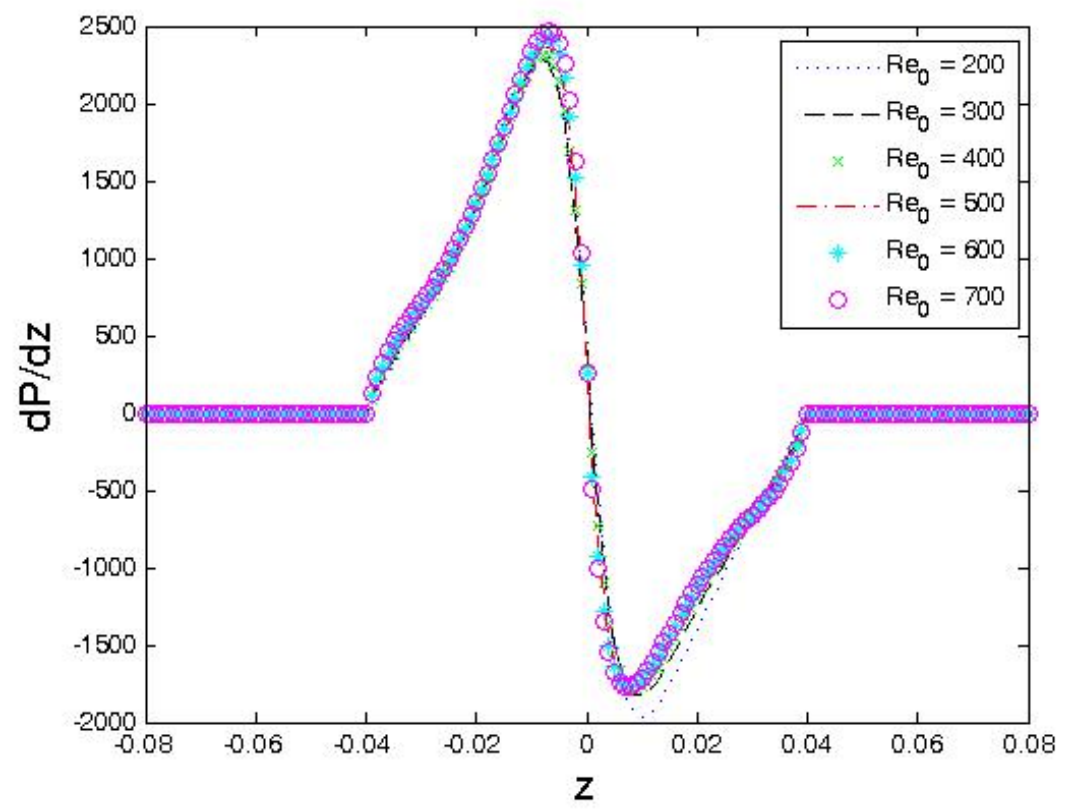

Figure 4.27: Pressure gradient curves in Piece-wise Cosinusoidal Geometry with $\delta=0.3, w_{s}=0.1$ and $M a_{0}=0.1$ as a function of $z$ for various Reynolds numbers.

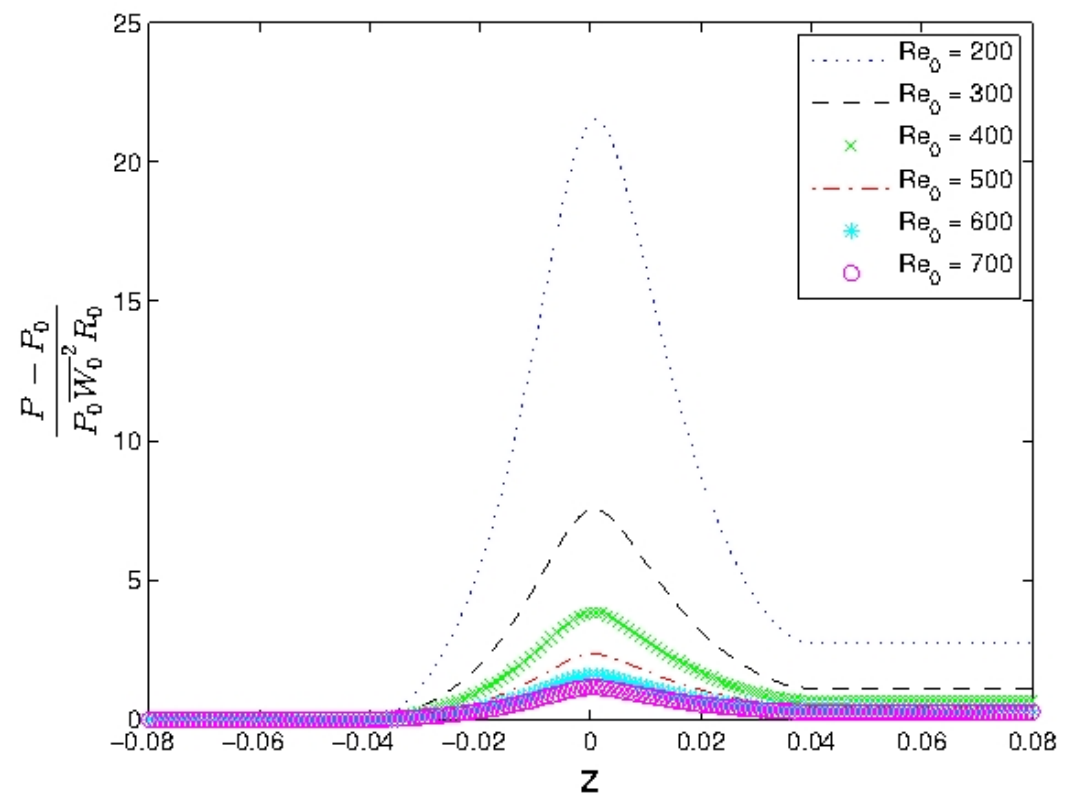

Figure 4.28: Pressure curves in Piece-wise Cosinusoidal Geometry with $\delta=0.3, w_{s}=0.1$ and $M a_{0}=0.1$ as a function of $z$ for various Reynolds numbers. 


\subsection{Piece-wise Polynomial Geometry}

\subsubsection{Comparison with MPC results and existing theoretical curves}

No-slip

In this section we compare the MPC and theoretical results from Akhter and Rohlf's density curves [1] with ours. The correlation between pressure and density is:

$$
P(z)=\frac{k_{B} T}{m} \rho(z)
$$

and we set $\frac{k_{B} T}{m}=1$ for agreement with the MPC results, therefore $P(z)=\rho(z)$.

We implement the geometry from Akhter and Rohlf [1], namely, a Piece-wise Polynomial Geometry in our analytical approach and we obtain the density curves by choosing $\delta=0.5$ and fixing $g=0.01,0.02,0.005$.

In Figures 4.29, 4.30 and 4.31, with no-slip $w_{s}=0$, we notice that there is an overlap between our curves and the theoretical curves from [1]. However, both curves have less dip and lower peak magnitude where the MPC result has more dip and a larger peak magnitude. At $g=0.02$, our density curve has a similar maximum peak but much lower dip in comparison to the MPC and theoretical density curves from [1]. As $g$ decreases from 0.01 to 0.005 we observe more agreement between the MPC and theoretical results from [1] and our density curve.

\section{Slip}

Maintaining the same structure and using the flow geometry picked in the previous subsection, varying the slip to $w_{s}=0.5$, setting $\delta=0.5$ and fixing $g=0.01,0.02,0.005$, we plot density curves and compare our results to the MPC and theoretical density curves from [1]. It is worth noting here that $M a$ for MPC curves is much larger than our $M a_{0}$ used in this research. Hence, MPC curves are not as smooth.

We see from Figures 4.29, 4.30 and 4.31 that for the case of $g=0.01$, there is an overlap between Akhter's theorical density curve [1] and ours. However, both curves have a lower peak and less dip in comparison to the MPC curve. Using $g=0.02$, we observe that our density curve has the same peak value comparing to Akhter's theoretical density curve, but much more dip which results in a density value less than 1. Furthermore, our density curve has a higher peak than the MPC density curve. At $g=0.005$, our density curve has much better agreement with Akhter's MPC density curve but less in magnitude, and our density curve overlaps with Akhter's theoretical curve. 


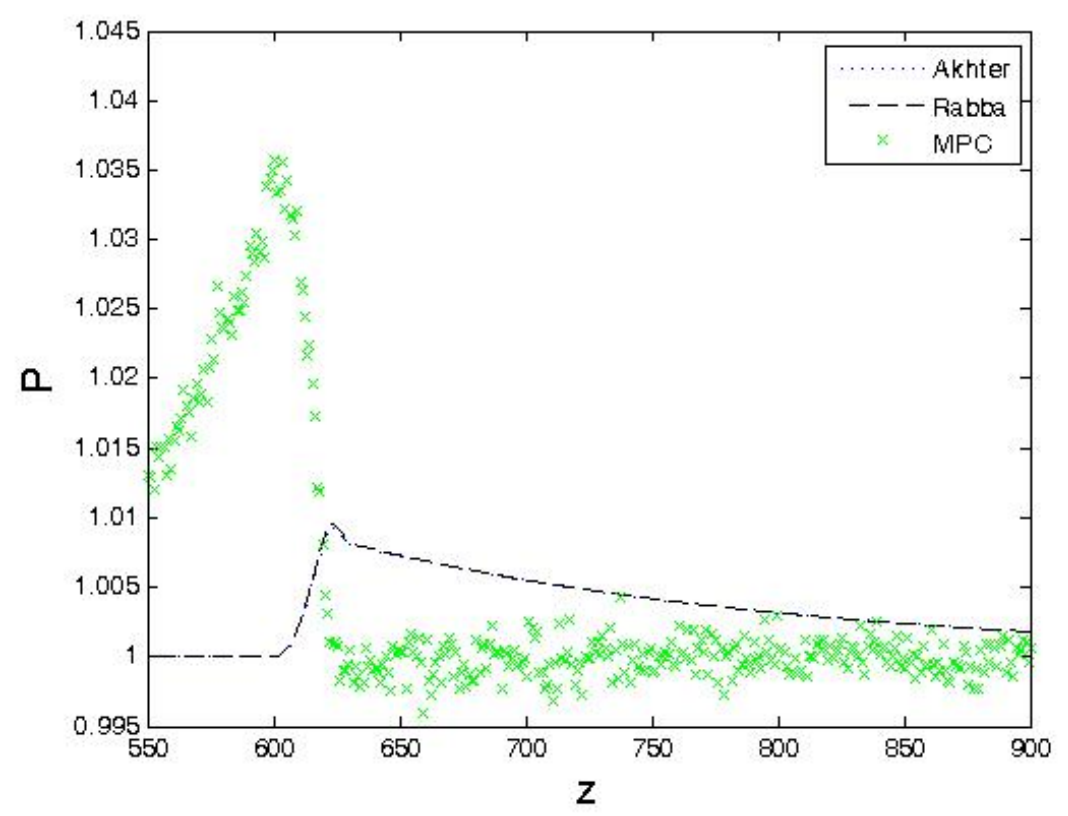

Figure 4.29: My Pressure curves in Piece-wise Polynomial Geometry with $\delta=0.5, l_{1}=20, l_{2}=10, w_{s}=0$ and $g=0.01$ as a function of $z$ and Comparison with MPC results and theoretical curves (labeled Akhter) from [1]. 


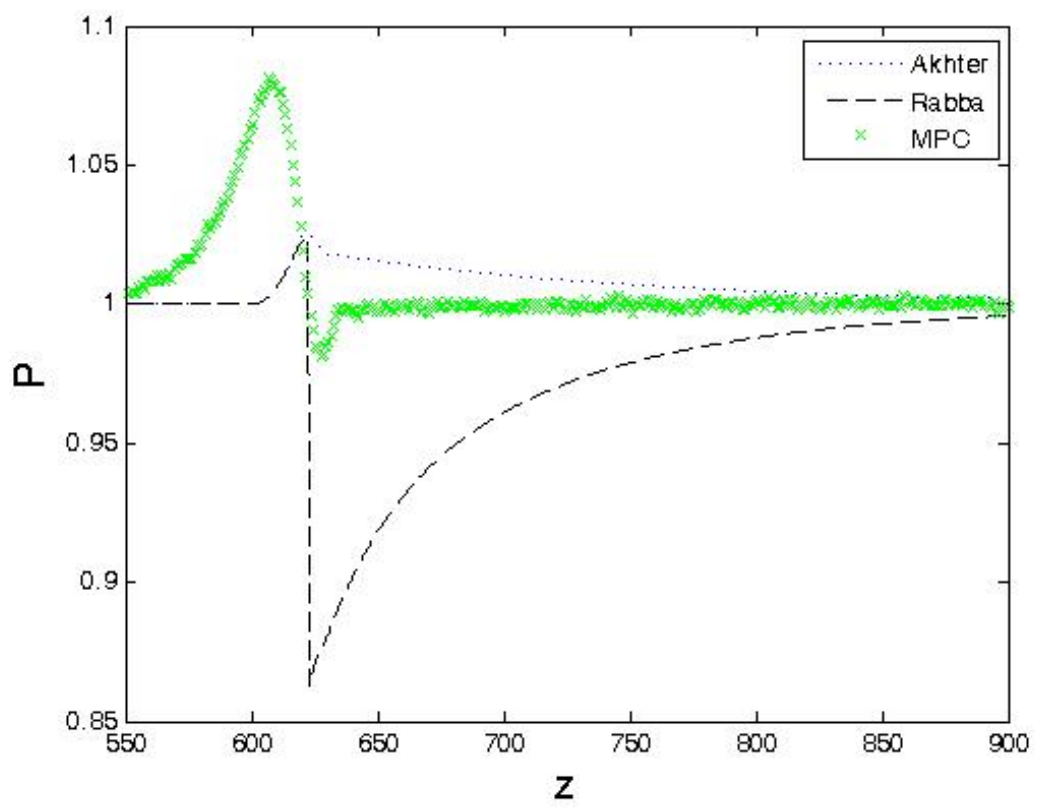

Figure 4.30: My Pressure curves in Piece-wise Polynomial Geometry with $\delta=0.5, l_{1}=20, l_{2}=10, w_{s}=0$ and $g=0.02$ as a function of $z$ and Comparison with Akhter's MPC results and her theoretical curves.

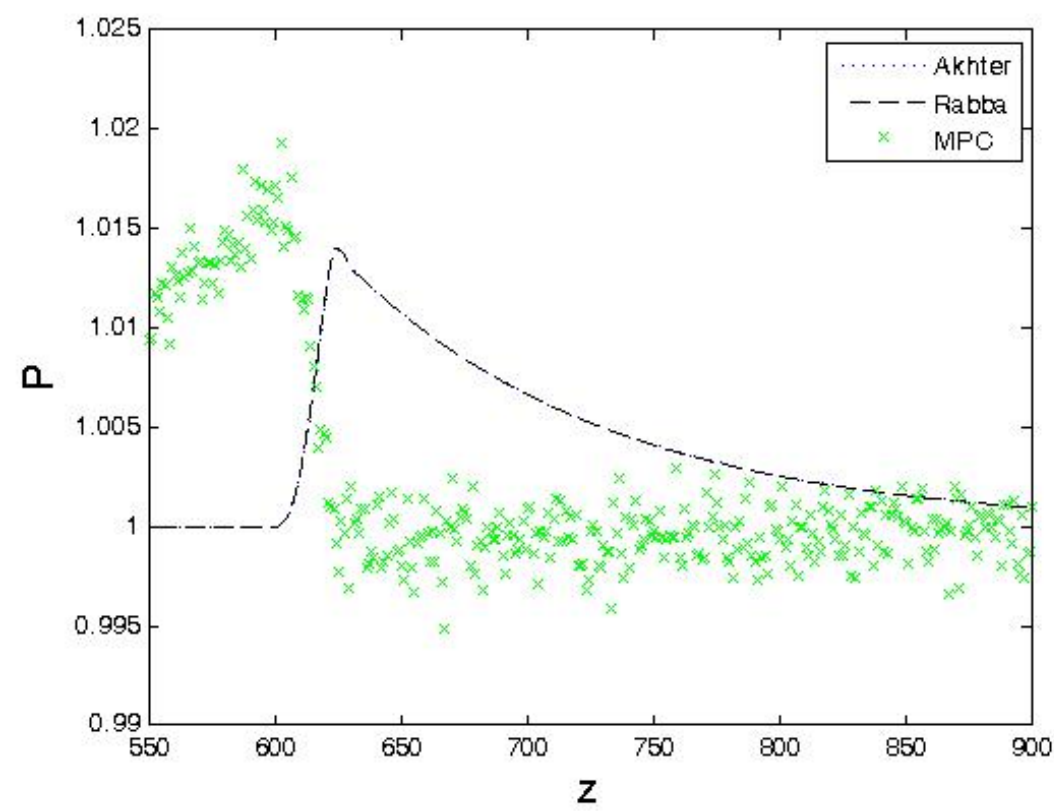

Figure 4.31: My Pressure curves in Piece-wise Polynomial Geometry with $\delta=0.5, l_{1}=20, l_{2}=10, w_{s}=0$ and $g=0.005$ as a function of $z$ and Comparison with Akhter's MPC results and her theoretical curves. 


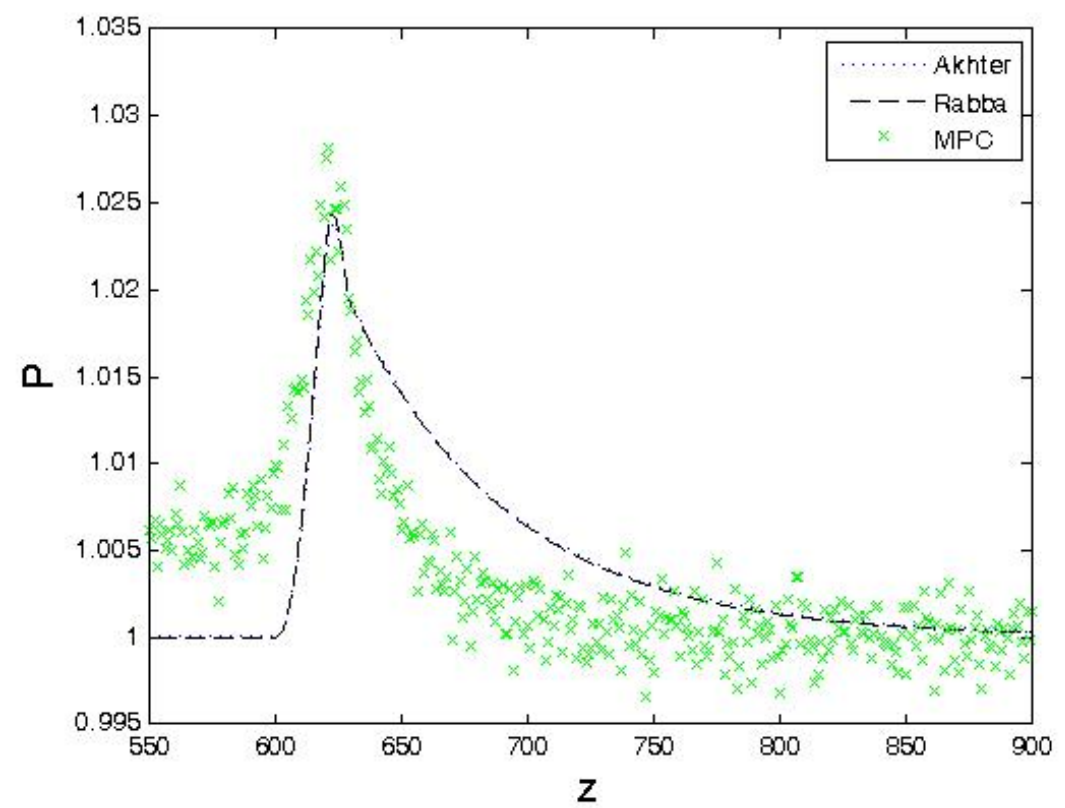

Figure 4.32: My Pressure curves in Piece-wise Polynomial Geometry with $\delta=0.5, l_{1}=20, l_{2}=10, w_{s}=0.5$ and $g=0.01$ as a function of $z$ and Comparison with Akhter's MPC results and her theoretical curves

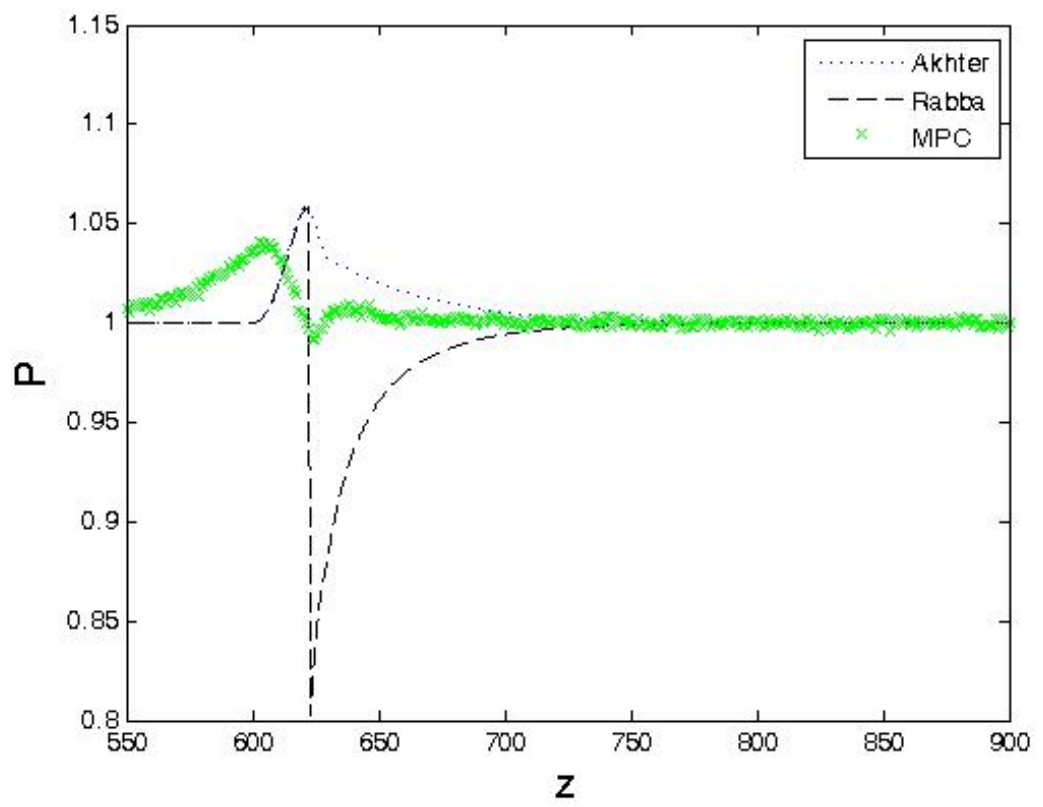

Figure 4.33: My Pressure curves in Piece-wise Polynomial Geometry with $\delta=0.5, l_{1}=20, l_{2}=10, w_{s}=0.5$ and $g=0.02$ as a function of $z$ and Comparison with Akhter's MPC results and her theoretical curves 


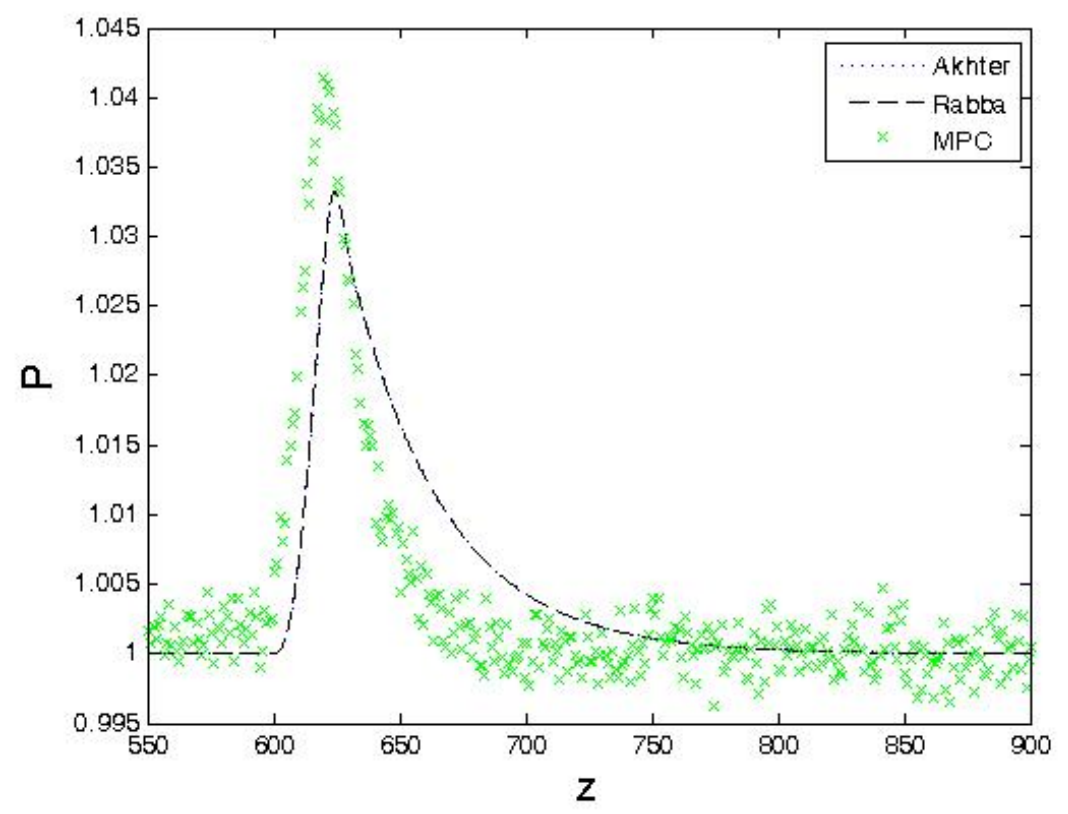

Figure 4.34: My Pressure curves in Piece-wise Polynomial Geometry with $\delta=0.5, l_{1}=20, l_{2}=10, w_{s}=0.5$ and $g=0.005$ as a function of $z$ and Comparison with Akhter's MPC results and her theoretical curves 


\section{Chapter 5}

\section{Conclusions and Future work}

Our analysis deals with an analytical investigation of blood flow pressure through a constriction in the presence of velocity slip and compressibility factors. We observed how Reynolds number has a great influence on the flow field in the constricted cylinder for a steady flow.

For the purpose of comparison, by disregarding the slip effects and by considering $M a_{0}$ to be very small, we varied the degree and the length of the constriction, then we assessed the role of velocity slip and compressibility in blood flow pressure and density curves. In addition, the analysis of broadening the axial width in the same stenotic degree $\delta$ indicates that the pressure and pressure gradient are more influenced in the constricted area.

We also implemented a Gaussian geometry and compared the results to existing results in the literature[9]. We extended the comparison against two other geometries, namely, piece-wise Cosinusoidal and polynomial geometries.

This analysis sustains the potential to further explore the pressure gradient and pressure curves and improvements may be obtained when the second-order derivatives are kept as well in the pressure gradient equations, which can be assessed and analyzed in the same context. 


\section{Appendix A}

\section{Appendix}

In this Appendix, we develop some relationships resulting from the velocity distribution (3.4)-(3.11). In the first section, we provide a relationship between centerline velocity $W$ and average velocity $\bar{W}$ using the definition of flow rate, and in the second section, we show that flow rate is constant.

\section{A.1 $W$ in terms of $\bar{W}$}

By definition, the flow rate is given by

$$
Q=\pi \rho R^{2} \bar{W}=\int_{0}^{R} 2 \pi \rho w r d r
$$

where $\bar{W}$ is the mean velocity at a given cross-section of the cylinder.

Substituting (3.4) for $w$, using (3.5)-(3.11), and solving for $W$ in terms of $\bar{W}$, gives the relationship

$$
W=\frac{2}{97} \frac{R^{2} \frac{d P}{d z}}{\mu}+\frac{210}{97} \bar{W}-\frac{102}{97} \frac{w_{s}}{\sqrt{1+R^{\prime 2}}}-\frac{2}{97} \frac{\rho g R^{2}}{\mu}-\frac{11}{97} w_{s} .
$$

This is one result needed throughout Chapter 3.

\section{A.2 Constant flow rate}

Another needed result throughout Chapter 3 is that flow rate is constant. To see this, differentiate $Q$ with respect to $z$ using the integral definition in (A.1) to get

$$
\frac{d Q}{d z}=2 \pi \frac{d}{d z} \int_{0}^{R} r \rho w d r=2 \pi\left[\left.(r \rho w)\right|_{r=R} R^{\prime}+\int_{0}^{R} r \frac{d}{d z}(\rho w) d r\right] .
$$


Now from the continuity equation (3.1),

$$
\frac{\partial}{\partial z}(\rho w)=-\frac{\rho}{r} \frac{\partial}{\partial r}(r u)
$$

Thus

$$
\frac{d Q}{d z}=2 \pi\left[\left.(r \rho w)\right|_{r=R} R^{\prime}-\int_{0}^{R} \rho \frac{d}{d r}(r u) d r\right] .
$$

Since $\rho=\rho(z)$, this can be integrated to get

$$
\frac{d Q}{d z}=2 \pi\left[\left.(r \rho w)\right|_{r=R} R^{\prime}-\rho\left[(r u)_{r=R}-(r u)_{r=0}\right]\right]=2 \pi \rho R\left(R^{\prime} w_{r=R}-\left.u\right|_{r=R}\right) .
$$

Now

$$
\left.u\right|_{r=R}=\frac{w_{s} R^{\prime}}{\sqrt{1+R^{\prime 2}}} \quad \text { and }\left.\quad w\right|_{r=R}=\frac{w_{s}}{\sqrt{1+R^{\prime 2}}}
$$

Thus,

$$
\frac{d Q}{d z}=2 \pi \rho R\left[\frac{w_{s} R^{\prime}}{\sqrt{1+R^{\prime 2}}}-\frac{w_{s} R^{\prime}}{\sqrt{1+R^{\prime 2}}}\right]=0
$$

implying flow rate is constant.

using $Q=2 \pi \int_{0}^{R} r \rho w d r$ and $\frac{d Q}{d z}=0$ together to get an expression for $\int_{0}^{R} r \frac{d w}{d z} d r$ in terms of $\rho, \frac{d \rho}{d z}$ and $\int_{0}^{R} r w d r$ we get

$$
\frac{d}{d z} \int_{0}^{R} r w d r=\left.(r w)\right|_{r=R} R^{\prime}+\int_{0}^{R} r \frac{d w}{d z} \Rightarrow \int_{0}^{R} r \frac{d w}{d z} d r=\frac{d}{d z} \int_{0}^{R} r w d r-\left.r w\right|_{r=R} R^{\prime}=\frac{d}{d z}\left[\frac{Q}{2 \pi \rho}\right]-\frac{R w_{s}}{\sqrt{1+R^{\prime 2}}} R^{\prime}
$$

hence;

$$
\frac{d}{d z} \int_{0}^{R} r w d r=-\frac{Q}{2 \pi \rho^{2}} \frac{d \rho}{d z}-\frac{R w_{s}}{\sqrt{1+R^{\prime 2}}} R^{\prime}
$$




\section{Appendix B}

\section{Appendix}

In this Appendix, we provide some relationships between derivatives of integrals and corresponding integrals with derivatives of integrands. These results are needed in the derivation of the Pressure Gradient equation in Chapter 3.

\section{B.0.1 Derivatives of integrals involving $w$}

Some derivatives of integrals involving $w$ will be needed in the derivation of the pressure gradient equation in Chapter 3. They are as follows.

$$
\begin{gathered}
\frac{d}{d z} \int_{0}^{R} r w d r=\left.(r w)\right|_{r=R} R^{\prime}+\int_{0}^{R} r \frac{d w}{d z} d r=\frac{R w_{s}}{\sqrt{1+R^{\prime 2}}} R^{\prime}+\int_{0}^{R} r \frac{d w}{d z} d r \\
\frac{d}{d z} \int_{0}^{R} r \rho w d r=\left.(r \rho w)\right|_{r=R} R^{\prime}+\int_{0}^{R} r \frac{d}{d z}(\rho w) d r=\frac{R \rho w_{s}}{\sqrt{1+R^{\prime 2}}} R^{\prime}+\int_{0}^{R} r \frac{d}{d z}(\rho w) d r \\
\frac{d}{d z} \int_{0}^{R} r w^{2} d r=\frac{R R^{\prime} w_{s}^{2}}{\left(1+R^{\prime 2}\right)}+\int_{0}^{R} r \frac{d}{d z} w^{2} d r
\end{gathered}
$$

Differentiate (B.1) using $\frac{d w_{s}}{d z}=0$ to get

$$
\begin{aligned}
\frac{d^{2}}{d z^{2}} \int_{0}^{R} r w d r= & \frac{d}{d z}\left[\frac{R w_{s}}{\sqrt{1+R^{\prime 2}}} R^{\prime}\right]+\frac{d}{d z} \int_{0}^{R} r \frac{d w}{d z} d r \\
= & \frac{R w_{s}}{\sqrt{1+R^{\prime 2}}} \frac{d^{2} R}{d z^{2}}+R^{\prime}\left[\frac{R}{\sqrt{1+R^{\prime 2}}} \frac{d w_{s}}{d z}+w_{s} \cdot \frac{\sqrt{1+R^{\prime 2}}-2 R R^{\prime} R^{\prime \prime} \frac{1}{2}\left(1+R^{\prime 2}\right)^{-\frac{1}{2}}}{\left(1+R^{\prime 2}\right)}\right] \\
\quad+\left.R \frac{d w}{d z}\right|_{r=R} R^{\prime}+\int_{0}^{R} r \frac{d^{2} w}{d z^{2}} d r & \\
= & \left.\frac{R w_{s}}{\sqrt{1+R^{\prime 2}}} \frac{d^{2} R}{d z^{2}}+\frac{w_{s}}{\sqrt{1+R^{\prime 2}}} R^{\prime}-\frac{R w_{s}}{\left(1+R^{\prime 2}\right)^{\frac{3}{2}}}\left(R^{\prime}\right)^{2} \frac{d R^{2}}{d z^{2}}-\frac{R w_{s}}{\left(1+R^{\prime 2}\right)^{\frac{3}{2}}} R^{\prime 2} \frac{d^{2} R}{d z^{2}}+\int_{0}^{R} r \frac{d^{2} w}{d z^{2}} \cdot d \eta\right)
\end{aligned}
$$


Thus

$$
\frac{d^{2}}{d z^{2}} \int_{0}^{R} r w d r=\int_{0}^{R} r \frac{d^{2} w}{d z^{2}} d r+\frac{R w_{s}}{\sqrt{1+R^{\prime 2}}} \frac{d^{2} R}{d z^{2}}+\frac{R^{\prime} w_{s}}{\sqrt{1+R^{\prime 2}}}-\frac{2 R R^{\prime 2} w_{s}}{\left(1+R^{\prime 2}\right)^{\frac{3}{2}}} \frac{d^{2} R}{d z^{2}}
$$

Differentiate (B.2) to get

$$
\begin{aligned}
\frac{d^{2}}{d z^{2}} \int_{0}^{R}(r \rho w) d r= & {\left[\frac{\rho\left[R R^{\prime \prime}+R^{\prime 2}\right] w_{s}}{\sqrt{1+R^{\prime 2}}}+\frac{R w_{s} R^{\prime}}{\sqrt{1+R^{\prime 2}}} \frac{d \rho}{d z}-\frac{R \rho w_{s} R^{\prime 2} R^{\prime \prime}}{\left(1+R^{\prime 2}\right)^{3 / 2}}\right] } \\
+ & {\left[\frac{R w_{s} R^{\prime}}{\sqrt{1+R^{\prime 2}}} \frac{d \rho}{d z}-\frac{R \rho w_{s} R^{\prime 2} R^{\prime \prime}}{\left(1+R^{\prime 2}\right)^{3 / 2}}+\int_{0}^{R} r \frac{d^{2}}{d z^{2}}(\rho w) d r\right] }
\end{aligned}
$$

Thus

$$
\frac{d^{2}}{d z^{2}} \int_{0}^{R}(r \rho w) d r=\frac{\rho\left[R R^{\prime \prime}+R^{\prime 2}\right] w_{s}}{\sqrt{1+R^{\prime 2}}}+\frac{2 R w_{s} R^{\prime}}{\sqrt{1+R^{\prime 2}}} \frac{d \rho}{d z}-\frac{2 R \rho w_{s} R^{\prime 2} R^{\prime \prime}}{\left(1+R^{\prime 2}\right)^{3 / 2}}+\int_{0}^{R} r \frac{d^{2}}{d z^{2}}(\rho w) d r
$$




\section{Appendix C}

\section{Appendix}

In this Appendix we obtain an expression for $\frac{d}{d z} \int_{0}^{R} r w^{2} d r$ using the axial velocity expression derived in Section 3.1 with the help of Maple.

$$
\begin{array}{rl}
\int_{0}^{R} r w^{2} & d r=-\frac{1}{7903560} \frac{\left(-305 R^{8} \pi^{2} \rho^{2}-305 R^{8} \pi^{2} \rho^{2} R^{\prime 2}\right)}{R^{2}\left(1+R^{\prime 2}\right) \mu^{2} \rho^{2} \pi^{2}}\left(\frac{d P}{d z}\right)^{2} \\
& -\frac{1}{7903560} \frac{1}{R^{2}\left(1+R^{\prime 2}\right) \mu^{2} \rho^{2} \pi^{2}}\left[1900 w_{s} \pi^{2} \rho^{2} R^{6} \mu+\left(35960 w_{s} \pi^{2} \rho^{2} R^{6} \mu \sqrt{1+R^{\prime 2}}\right)\right. \\
& \left.+610 R^{8} \pi^{2} \rho^{3} g+610 R^{8} \pi^{2} \rho^{3} g R^{\prime 2}+1900 w_{s} \pi^{2} \rho^{2} R^{6} R^{\prime 2}-37860 R^{4} \pi \rho Q \mu-37860 R^{4} \pi \rho Q \mu R^{\prime 2}\right]\left(\frac{d P}{d z}\right) \\
& -\frac{1}{7903560} \frac{1}{R^{2}\left(1+R^{\prime 2}\right) \mu^{2} \rho^{2} \pi^{2}}\left[3098740 w_{s} \pi \rho R^{2} \mu^{2} Q \sqrt{1+R^{\prime 2}}-1505272 w_{s}^{2} \pi^{2} \rho^{2} R^{4} \mu^{2}\right. \\
& -35960 w_{s} \pi^{2} \rho^{3} R^{6} \mu g \sqrt{1+R^{\prime 2}}+102500 w_{s} \pi \rho R^{2} \mu^{2} Q+102500 w_{s} \pi \rho R^{2} \mu^{2} Q R^{\prime 2}-305 \rho^{4} g^{2} R^{8} \pi^{2} \\
& -3576 w_{s}^{2} \pi^{2} \rho^{2} R^{4} \mu^{2} R^{\prime 2}-1900 w_{s} \pi^{2} \rho^{3} R^{6} \mu g R^{\prime 2}-95348 w_{s}^{2} \pi^{2} \rho^{2} R^{4} \mu^{2} \sqrt{1+R^{\prime 2}} \\
& \left.-305 \rho^{4} g^{2} R^{8} \pi^{2} R^{\prime 2}+37860 Q \mu \rho^{2} g R^{4} \pi+37860 Q \mu \rho^{2} R^{4} R^{\prime 2}-5552400 Q^{2} \mu^{2}-5552400 Q^{2} \mu^{2} R^{\prime 2}\right] \quad(\mathrm{C} .1)
\end{array}
$$

Note that (A.2) has been used to replace $W$ in terms of $\bar{W}$, and $Q=2 \pi \rho \bar{W}$ has been used to replace $\bar{W}$ in terms of $Q$.

Simplifying the above equation, we get

$$
\begin{array}{rl}
\int_{0}^{R} r w^{2} & d r=\frac{61}{1580712} \frac{R^{6}}{\mu^{2}}\left(\frac{d P}{d z}\right)^{2}-\frac{95}{395178} \frac{w_{s} R^{4}}{\mu}\left(\frac{d P}{d z}\right)-\frac{899}{197589} \frac{w_{s} R^{4}}{\mu \sqrt{1+R^{\prime 2}}}\left(\frac{d P}{d z}\right)+\frac{631}{131726} \frac{R^{2} Q}{\mu \rho \pi}\left(\frac{d P}{d z}\right) \\
& -\frac{61}{790356} \frac{R^{6} \rho g}{\mu^{2}}\left(\frac{d P}{d z}\right)-\frac{154937}{395178} \frac{w_{s} Q}{\pi \rho \sqrt{1+R^{\prime 2}}}+\frac{188159}{987945} \frac{w_{s}^{2} R^{2}}{1+R^{\prime 2}}+\frac{899}{197589} \frac{w_{s} \rho R^{4} g}{\mu \sqrt{1+R^{\prime 2}}}-\frac{5125}{395178} \frac{w_{s} Q}{\rho \pi} \\
& +\frac{61}{1580712} \frac{R^{6} \rho^{2} g^{2}}{\mu^{2}}+\frac{149}{329315} \frac{w_{s}^{2} R^{2} R^{\prime 2}}{1+R^{\prime 2}}+\frac{95}{395178} \frac{w_{s} \rho R^{4} g}{\mu}+\frac{23837}{1975890} \frac{w_{s}^{2} R^{2}}{\sqrt{1+R^{\prime 2}}}-\frac{631}{131726} \frac{Q R^{2} g}{\mu \pi} \\
+ & \frac{6610}{9409} \frac{Q^{2}}{R^{2} \rho^{2} \pi^{2}}
\end{array}
$$


Now taking $\frac{d}{d z}$ of this equation, using $\frac{d Q}{d z}=0$ and $\frac{d w_{s}}{d z}=0$ we get

$$
\begin{array}{rl}
\frac{d}{d z} \int_{0}^{R} & r w^{2} d r=\frac{61}{263452} \frac{R^{5}\left(\frac{d P}{d z}\right)^{2} R^{\prime}}{\mu^{2}}+\frac{61}{790356} \frac{(R)^{6} \frac{d P}{d z}\left(\frac{d^{2} P}{d z^{2}}\right)}{\mu^{2}}-\frac{190}{197589} \frac{\left(R^{\prime} w_{s}\right)(R)^{3} \frac{d P}{d z}}{\mu} \\
& -\frac{95}{395178} \frac{w_{s}(R)^{4} \frac{d^{2} P}{d z^{2}}}{\mu}-\frac{3596}{197589} \frac{w_{s}(R)^{3} \frac{d P}{d z} R^{\prime}}{\mu \sqrt{1+\left(R^{\prime}\right)^{2}}}-\frac{899}{197589} \frac{w_{s}(R)^{4} \frac{d^{2} P}{d z^{2}}}{\mu \sqrt{1+\left(R^{\prime}\right)^{2}}}+\frac{899}{197589} \frac{w_{s}(R)^{4} \frac{d P}{d z} R^{\prime} R^{\prime \prime}}{\mu\left(1+\left(R^{\prime}\right)^{2}\right)^{3 / 2}} \\
& +\frac{631}{65863} \frac{R Q \frac{d P}{d z} R^{\prime}}{\mu \rho \pi}+\frac{631}{131726} \frac{(R)^{2} Q \frac{d^{2} P}{d z^{2}}}{\mu \rho \pi}-\frac{631}{131726} \frac{(R)^{2} Q \frac{d P}{d z} \frac{d \rho}{d z}}{\mu(\rho)^{2} \pi}-\frac{61}{131726} \frac{(R)^{5} \rho g \frac{d P}{d z} R^{\prime}}{\mu^{2}} \\
& -\frac{61}{790356} \frac{(R)^{6}\left(\frac{d \rho}{d z}\right) g \frac{d P}{d z}}{\mu^{2}}-\frac{61}{790356} \frac{(R)^{6} \rho g \frac{d^{2} P}{d z^{2}}}{\mu^{2}}+\frac{154937}{395178} \frac{w_{s} Q R^{\prime} R^{\prime \prime}}{\left(1+\left(R^{\prime}\right)^{2}\right)^{3 / 2}}+\frac{154937}{395178} \frac{w_{s} Q \frac{d \rho}{d z}}{\sqrt{1+\left(R^{\prime}\right)^{2}}(\rho)^{2} \pi} \\
& +\frac{376318}{987945} \frac{\left(w_{s}\right)^{2} R R^{\prime}}{1+\left(R^{\prime}\right)^{2}}-\frac{376318}{987945} \frac{\left(w_{s}\right)^{2}(R)^{2} R^{\prime} R^{\prime \prime}}{\left(1+\left(R^{\prime}\right)^{2}\right)^{2}}+\frac{899}{197589} \frac{w_{s}\left(\rho^{\prime}\right)(R)^{4} g}{\mu \sqrt{1+\left(R^{\prime}\right)^{2}}+\frac{3596}{197589} \frac{w_{s} \rho(R)^{3} g R^{\prime}}{\mu \sqrt{1+\left(R^{\prime}\right)^{2}}}} \\
& -\frac{899}{197589} \frac{w_{s} \rho(R)^{4} g R^{\prime} R^{\prime \prime}}{\mu\left(1+\left(R^{\prime}\right)^{2}\right)^{3 / 2}}+\frac{5125}{395178} \frac{w_{s} Q \rho^{\prime}}{(\rho)^{2} \pi} \frac{61}{263452} \frac{(R)^{5}(\rho)^{2} g^{2} R^{\prime}}{\mu^{2}}+\frac{61}{790356} \frac{(R)^{6} \rho g^{2} \frac{d}{d z} \rho}{\mu^{2}} \\
& +\frac{298}{329315} \frac{w_{s}^{2} R R^{\prime 3}}{1+R^{\prime 2}}+\frac{298}{329315} \frac{w_{s}^{2} R^{2} R^{\prime} R^{\prime \prime}}{\left(1+R^{\prime 2}\right)}-\frac{298}{329315} \frac{\left(w_{s}\right)^{2}(R)^{2}\left(R^{\prime}\right)^{3} R^{\prime \prime}}{\left(1+\left(R^{\prime}\right)^{2}\right)^{2}} \\
& +\frac{95}{395178} \frac{w_{s}\left(\rho^{\prime}\right)(R)^{4} g}{\mu}+\frac{190}{197589} \frac{w_{s} \rho(R)^{3} g R^{\prime}}{\mu}+\frac{23837}{987945} \frac{\left(w_{s}\right)^{2} R R^{\prime}}{\sqrt{1+\left(R^{\prime}\right)^{2}}}-\frac{23837}{1975890} \frac{\left(w_{s}\right)^{2}(R)^{2} R^{\prime} R^{\prime \prime}}{\left(1+\left(R^{\prime}\right)^{2}\right)^{3 / 2}} \\
& -\frac{631}{65863} \frac{R Q g R^{\prime}}{\mu \pi}-\frac{13220}{9409} \frac{(Q)^{2} R^{\prime}}{(R)^{3}(\rho)^{2} \pi^{2}}-\frac{13220}{9409} \frac{(Q)^{2} \rho^{\prime}}{(R)^{2}(\rho)^{3} \pi^{2}}
\end{array}
$$




\section{Appendix D}

\section{Appendix}

In this Appendix we provide the Maple code used to generate the numerical pressure curves. RK4 method was used to solve the Differential Equation.

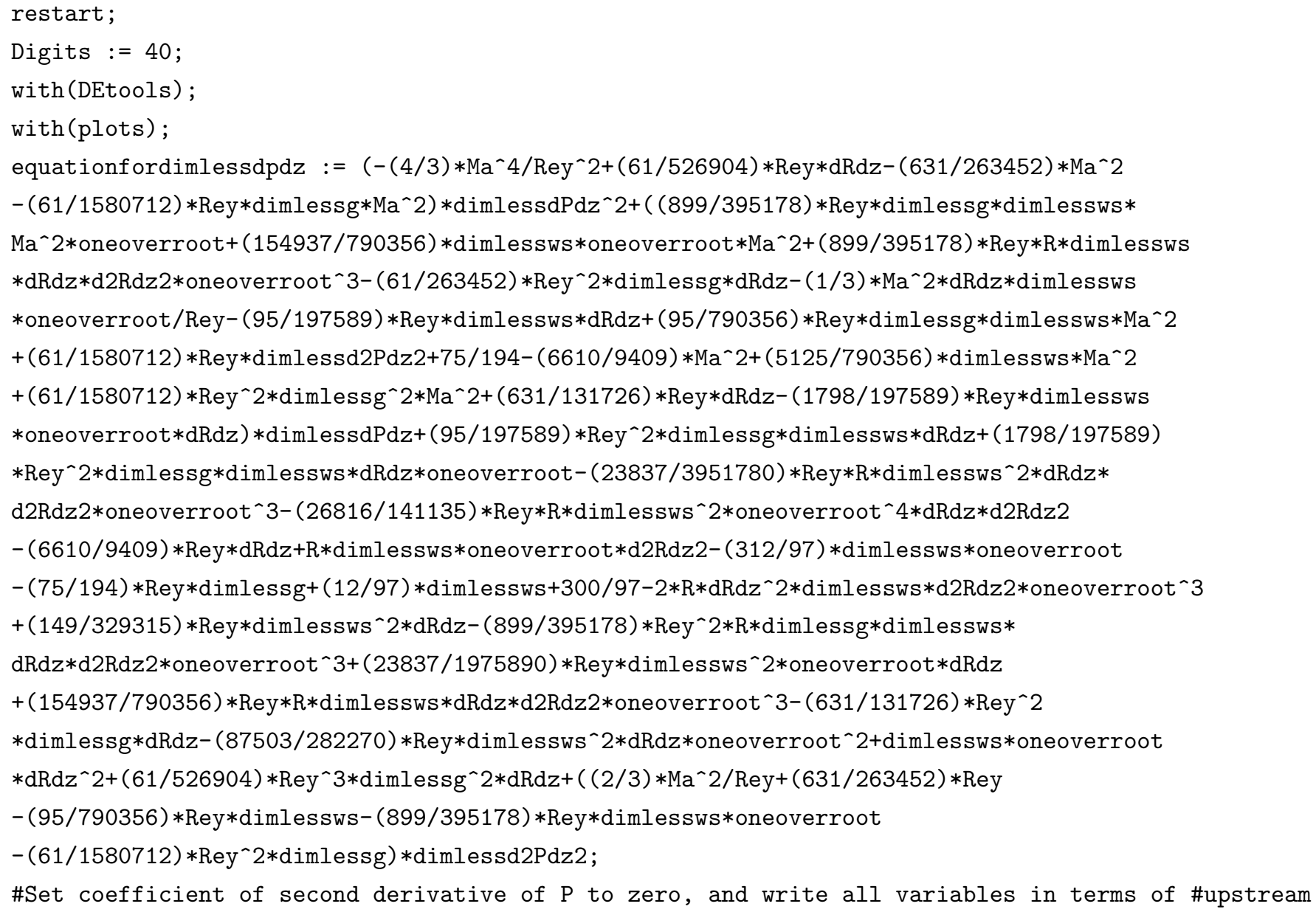




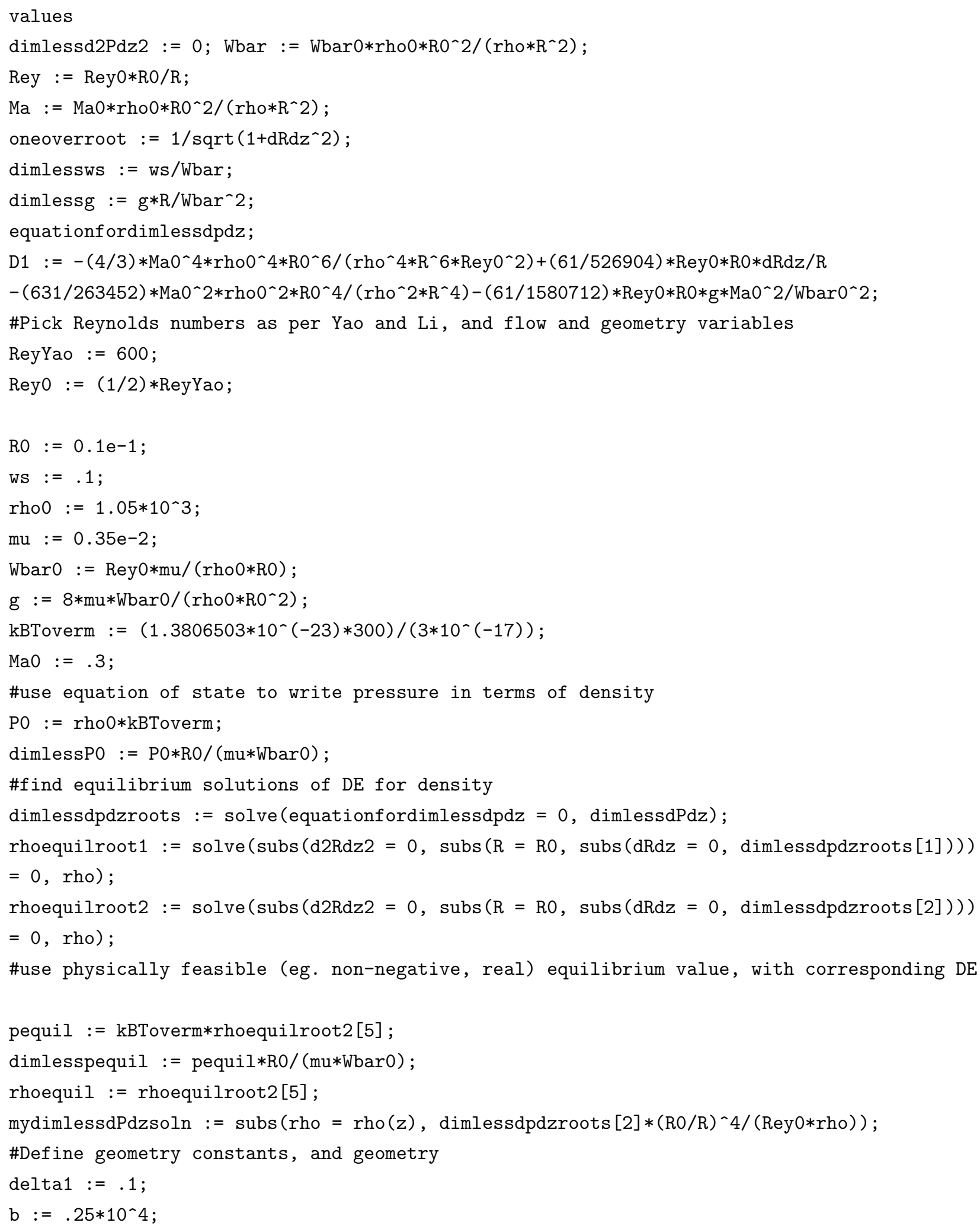




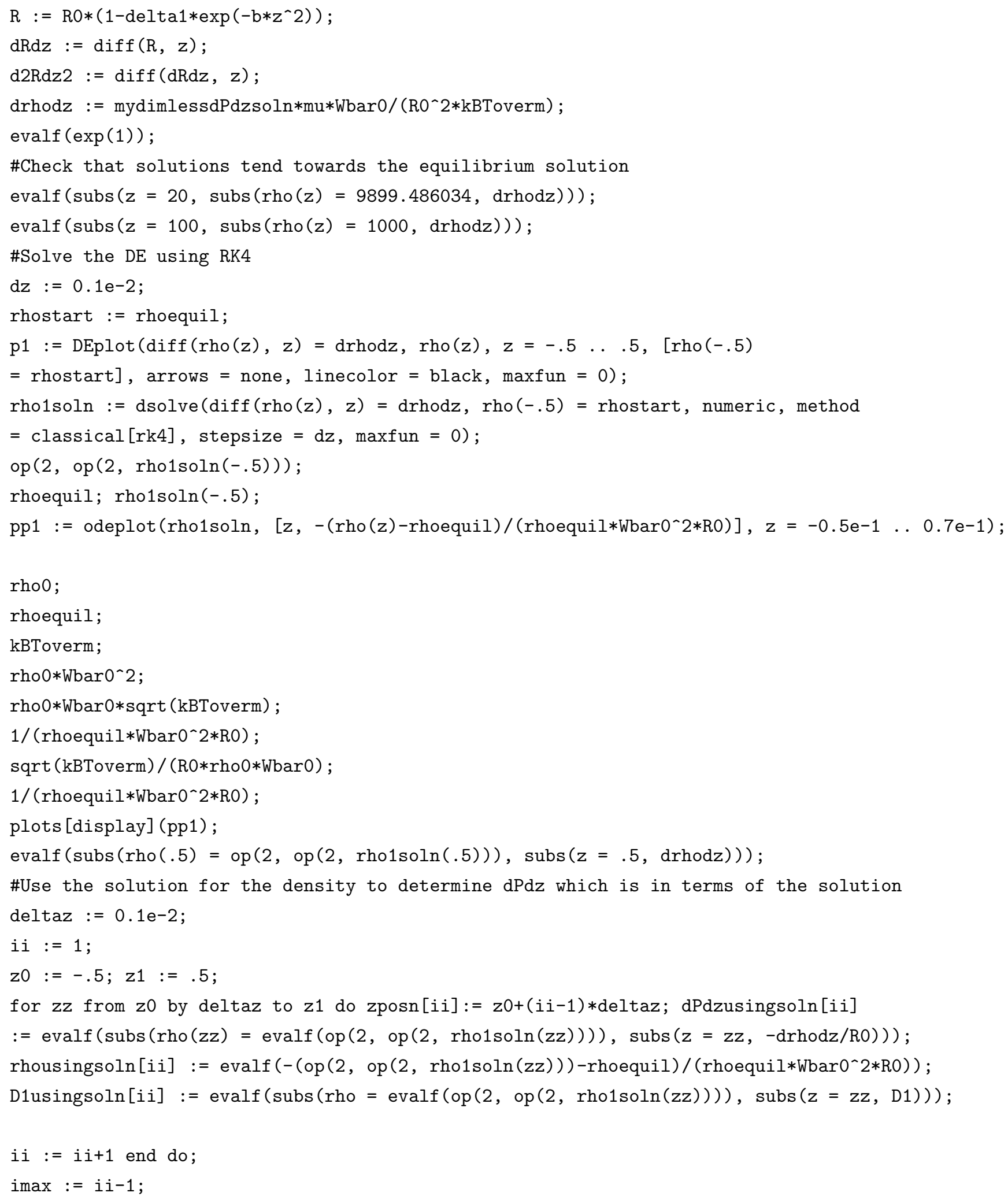




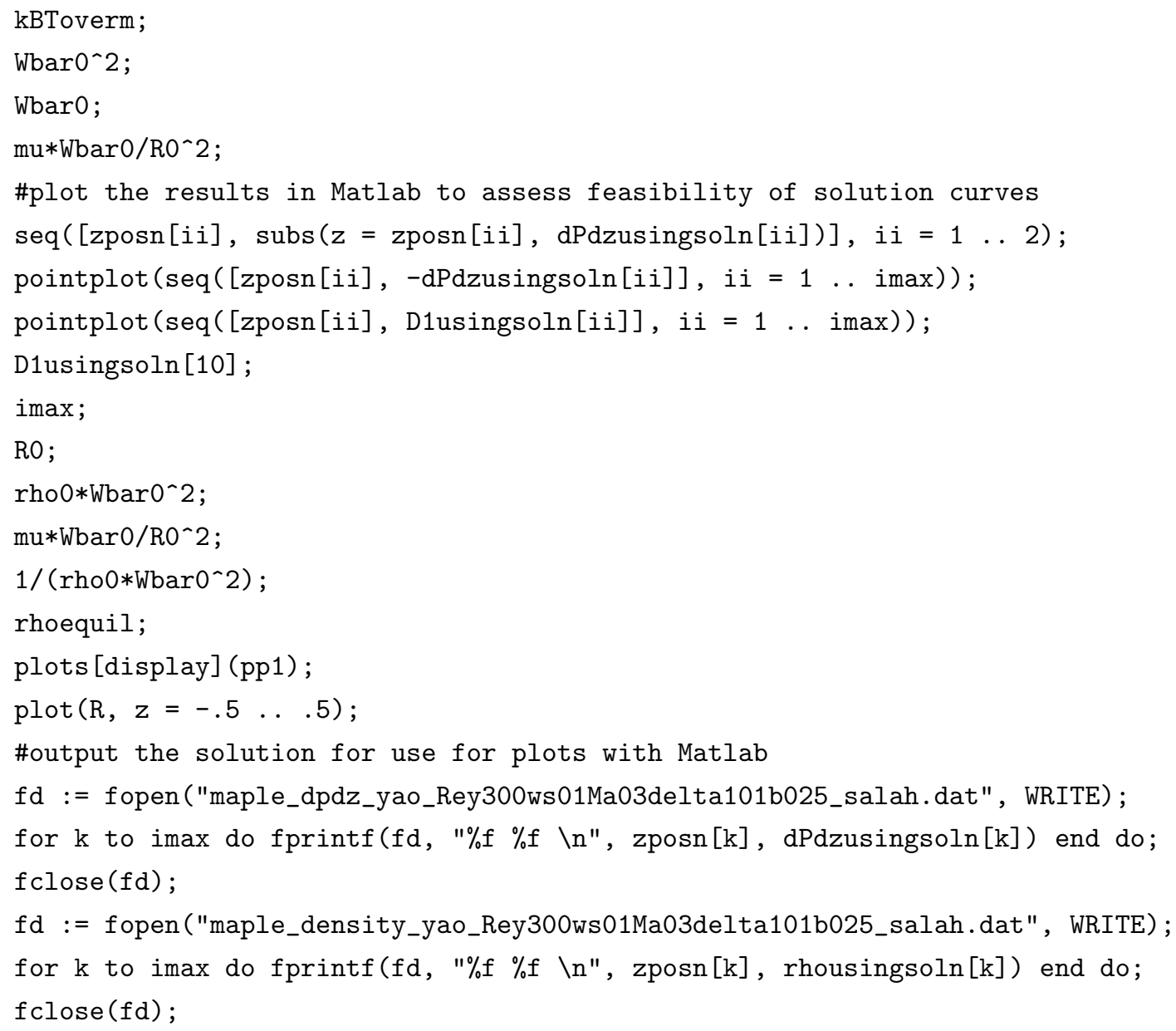




\section{References}

[1] T. Akhter and K. Rohlf. Quantifying compressibility and slip in multiparticle collision (MPC) flow through a local constriction. Entropy, 16:418-442, 2014.

[2] S. Bedkihal, J. C. Kumaradas, and K. Rohlf. Steady flow through a constricted cylinder by multiparticle collision dynamics. Biomechanics and Modeling in Mechanobiology, 12:929-939, 2013.

[3] S.A. Berger and L-D. Jou. Flows in stenotic vessels. Annu. Rev. Fluid Mech., 32:347-382, 2000.

[4] D. Biswas. Blood flow models: A Comparative Study. Mittal, 2004.

[5] J.R. Darias, M. Quiroga, E. Medina, P.J. Colmenares, and R. Paredes V. Simulation of suspensions in constricted geometries by dissipative particle dynamics. Molec. Sim., 29:443-449, 2003.

[6] S.J. Denardo, E.G. Yamada, V.K. Hargrave, and P.G. Yock. Effect of stenosis inlet geometry on shear rates of blood flow in the upstream region. American Heart Journal, 125:350-356, 1993.

[7] M.D. Deshpande, D.P. Giddens, and R.F. Mabon. Steady laminar flow through modelled vascular stenoses. J. Biomechan., 9:165-174, 1976.

[8] J. H. Forrester. Flow through a converging-diverging tube and it's implications in occlusive vascular disease. Ph. D. Thesis Ames, Iowa, Library, Iowa State University., 1968.

[9] J. H. Forrester and D. F. Young. Flow through a converging-diverging tube and it's implications in occlusive vascular disease-I: Theoretical development. J.Biomechanics., 3:297-385, 1970.

[10] J. H. Forrester and D. F. Young. Flow through a converging-diverging tube and it's implications in occlusive vascular disease-II: Theoretical and experimental results and their implications. J.Biomechanics., $3: 1-25,1970$.

[11] D. Hershey and S. J. Cho. Blood flow in rigid tubes, thickness and slip velocity of plasma film at the wall. Journal of Applied Physiology., 21:27-32, 1966.

[12] D.N. Ku. Blood flow in arteries. Annu. Rev. Fluid Mech., 29:399-434, 1997.

[13] J. S. Lee. Numerical study of fluid flow through double bell-shaped constrictions in a tube. International Journal of Numerical Methods for Heat \& Fluid Flow, 12:258-289, 2002. 
[14] J.-S. Lee and Y.-C. Fung. Flow in nonuniform small blood vessels. Microvasc. Res., 3:272-287, 1971.

[15] W. Liao, T. S. Lee, and H. T. Low. Numerical studies of physiological pulsatile flow through constricted tube. Emarald., 14:297-385, 2003.

[16] J.C. Misra and G.C. Shit. Role of slip velocity in blood flow through stenosed arteries: A non-Newtonian model. J. Mech. Med. Biol., 7:337-353, 2007.

[17] Y. Nubar. Blood flow, slip, and viscometry. Biophys. J., 11:252-264, 1971.

[18] N. Padmanabhan. Mathematical model of arterial stenosis. Med. ES Biol. Eng. E Computer, 18:281-226, 1980.

[19] G. Pontrelli. Blood flow through an axisymmetric stenosis. Proc. Instn. Mech. Engrs. Part H, 215:1-10, 2001.

[20] S.S. Varghese, S.H. Frankel, and P.F. Fischer. Direct numerical simulation of stenotic flows. part 1. steady flow. J. Fluid Mech., 582:253-280, 2007.

[21] L. Yao and D.-Z. Li. Pressure and pressure gradient in an axisymmetric rigid vessel with stenosis. Applied Mathematics and Mechanics (English Edition), 27:347-351, 2006.

[22] J. G. Zhou. Axisymmetric lattice Boltzmann method. Phys. Rev. E, 78:036701-1-7, 2008. 